\title{
Quality control of sealed beta sources in brachytherapy
}

Recommendations on detectors, measurement procedures

and quality control of beta sources

\section{NEDERLANDSE COMMISSIE VOOR STRALINGSDOSIMETRIE}

Report 14 of the Netherlands Commission on Radiation Dosimetry

\author{
Authors: \\ R.P. Kollaard \\ W.J.F. Dries \\ H.J. van Kleffens \\ A.H.L. Aalbers \\ J. van der Marel \\ J.P.A. Marijnissen \\ M. Piessens \\ D.R. Schaart \\ H. de Vroome
}

Netherlands Commission on Radiation Dosimetry

Task Group Quality control of sealed beta sources in brachytherapy August 2004 
https://doi.org/10.25030/ncs-014 The NCS report has been downloaded on 26 Apr 2023 


\section{Preface}

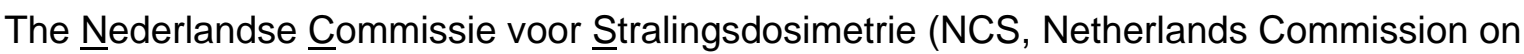
Radiation Dosimetry) was officially established on 3 September 1982 with the aim of promoting the appropriate use of dosimetry of ionizing radiation both for scientific research and practical applications. The NCS is chaired by a board of scientists, installed upon the suggestion of the supporting societies, including the Nederlandse Vereniging voor Radiotherapie en Oncologie (Netherlands Society for Radiotherapy and Oncology), the Nederlandse Vereniging voor Nucleaire Geneeskunde (Netherlands Society for Nuclear Medicine), the Nederlandse Vereniging voor Klinische Fysica (Netherlands Society for Clinical Physics), the Nederlandse Vereniging voor Radiobiologie (Netherlands Society for Radiobiology), the Nederlandse Vereniging voor Stralingshygiëne (Netherlands Society for Radiological Protection), the Nederlandse Vereniging voor Medische Beeldvorming en Radiotherapie (Netherlands Society for Medical Imaging and Radiotherapy), the Nederlandse Vereniging voor Radiologie (Netherlands Society for Radiology) and the Belgische Vereniging voor Ziekenhuisfysici/Société Belge des Physiciens des Hôpitaux (Belgian Hospital Physicists Association).

To pursue its aims, the NCS accomplishes the following tasks: participation in dosimetry standardisation and promotion of dosimetry intercomparisons, drafting of dosimetry protocols, collection and evaluation of physical data related to dosimetry. Furthermore the commission shall maintain or establish links with national and international organisations concerned with ionizing radiation and promulgate information on new developments in the field of radiation dosimetry.

Current members of the board of the NCS:

S. Vynckier, chairman B.J.M. Heijmen, vice-chairman W. de Vries, secretary J. Zoetelief, treasurer A.J.J. Bos A.A. Lammertsma J.M. Schut F.W. Wittkämper D. Zweers 


\title{
Quality Control of Sealed Beta Sources in Brachytherapy
}

This report was prepared by a task group of the Netherlands Commission on Radiation Dosimetry (NCS), consisting of Belgian and Dutch scientists. This work was financially supported by The Netherlands Organisation for Health Research and Development (ZonMw).

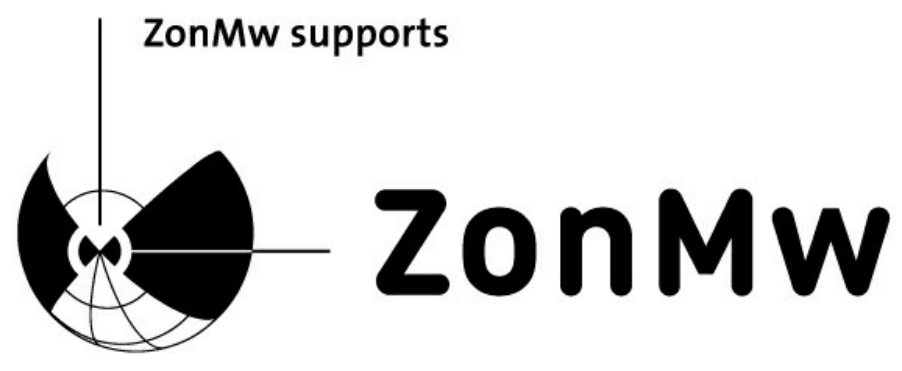

Members of the task group:

W.J.F. Dries, chairman

\author{
A.H.L. Aalbers \\ H.J. van Kleffens \\ R.P. Kollaard \\ J. van der Marel \\ J.P.A. Marijnissen \\ M. Piessens \\ D.R. Schaart \\ H. de Vroome
}




\section{User guide}

This report aims to provide information and guidelines for physicists who are responsible for the quality control of beta sources in radiotherapy. Chapters 1-4 give an introduction to the field of beta sources and dosimetry. The first three chapters give insight in the advantages and disadvantages of applying beta sources in brachytherapy and in the different systems that are available. Chapter 4 describes the current status of dosimetry of beta sources in the Netherlands and Belgium.

Chapter 5 can be of help in the practical implementation of dosimetrical quality control on beta sources. More details on practical measurement procedures are given in Appendix 10.

Chapter 6 and its summary in Chapter 7 can be used to implement a structural quality control program for beta sources. These chapters contain an overview of relevant quality control aspects, suitable equipment and recommendations on tolerances for deviations in source strength, non-uniformity and asymmetry. 


\section{Contents}

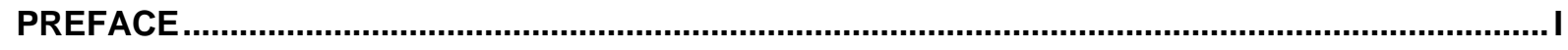

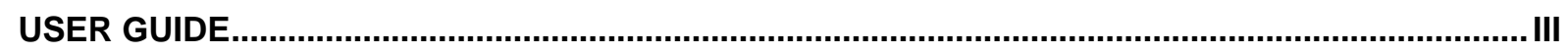

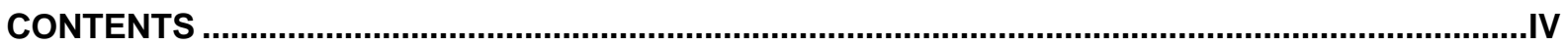

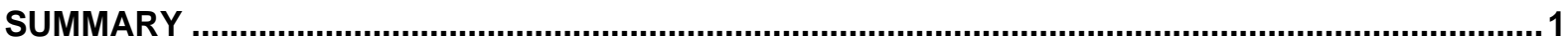

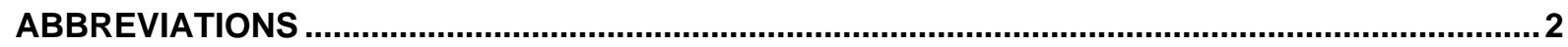

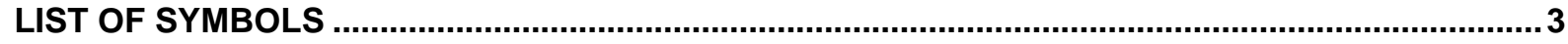

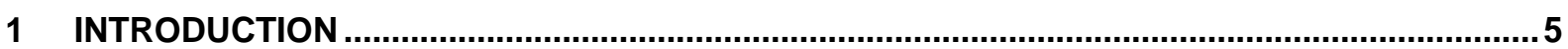

1.1 PURPOSE

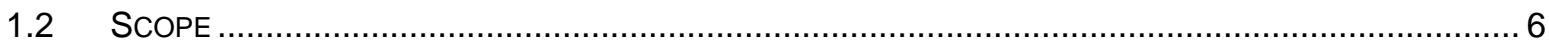

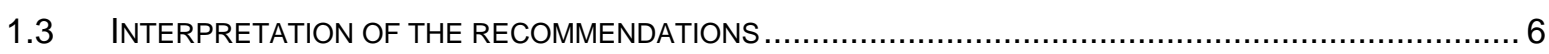

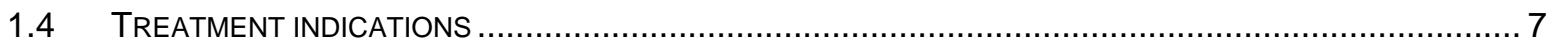

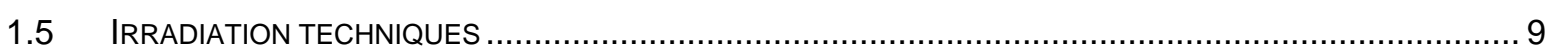

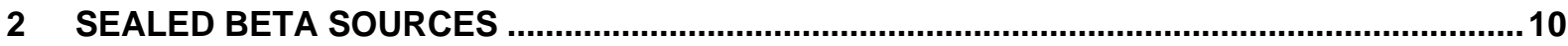

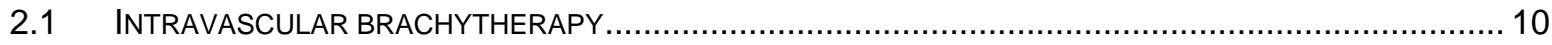

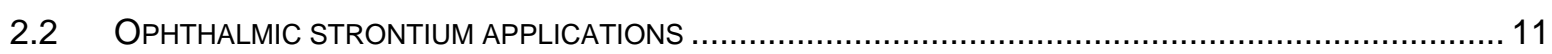

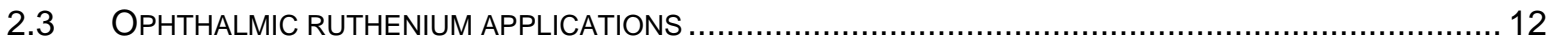

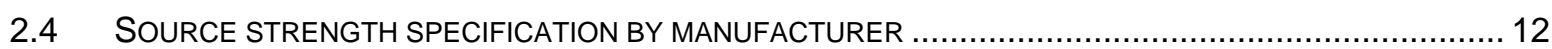

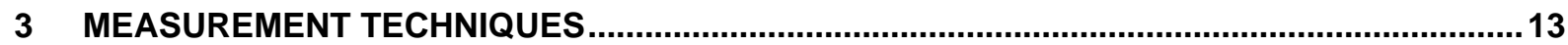

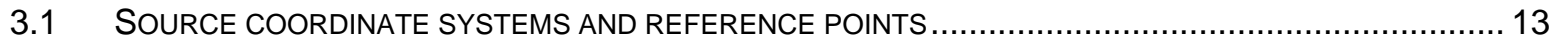

3.2 NCS DEFINITIONS OF DOSIMETRIC PARAMETERS FOR SEALED BETA SOURCES ............................. 13

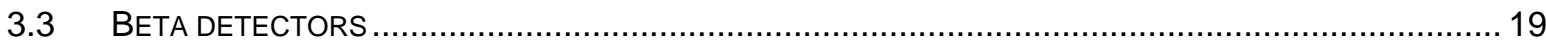

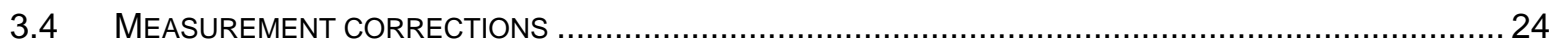

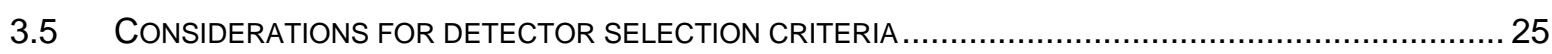

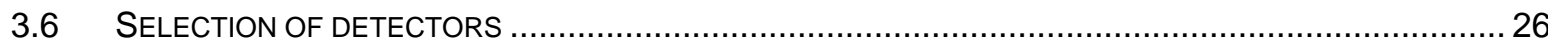

4 CURRENT STATUS OF DOSIMETRY OF BETA SOURCES …...........................................28

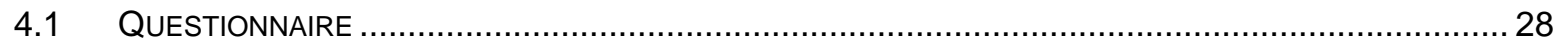

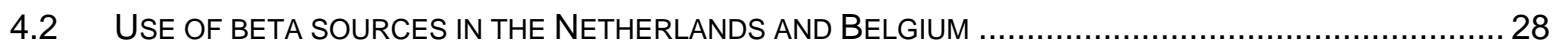

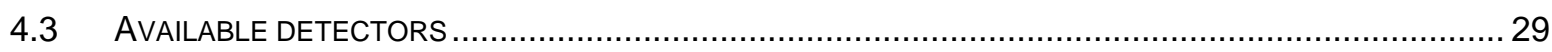

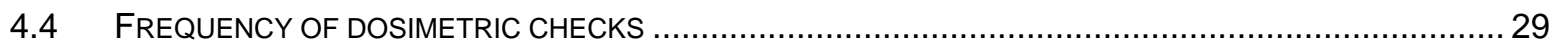

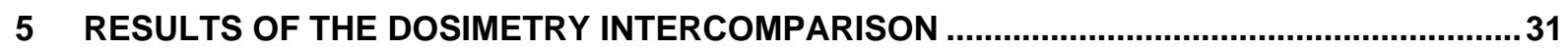

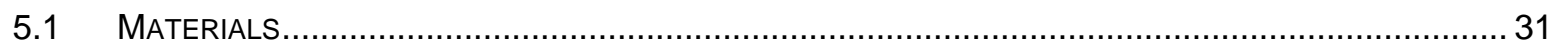

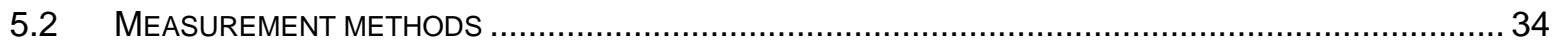

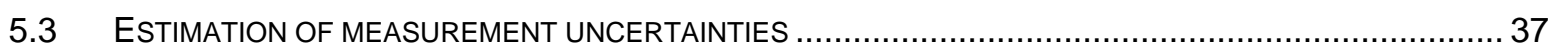




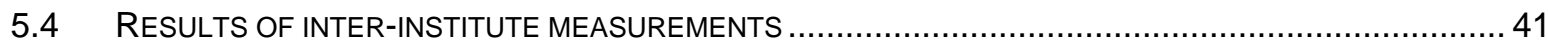

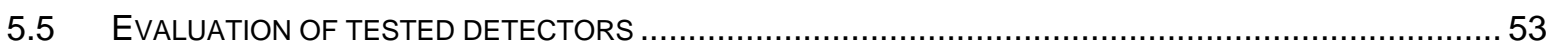

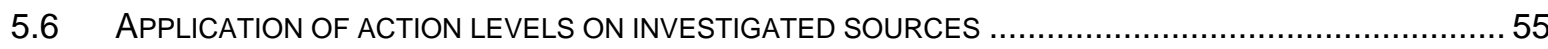

6 NCS RECOMMENDATIONS FOR QUALITY CONTROL OF BETA SOURCES ........................56

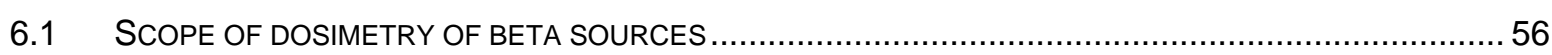

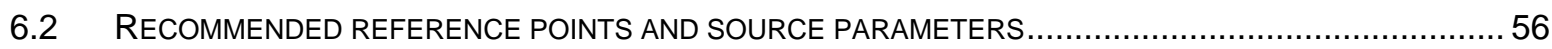

6.3 NCS RECOMMENDATIONS FOR SUITABLE MEASUREMENT SYSTEMS …........................................ 58

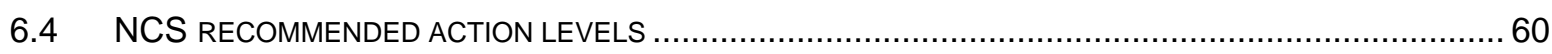

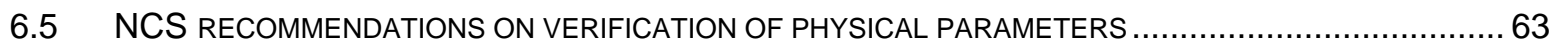

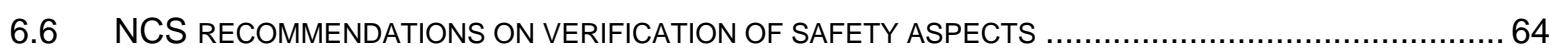

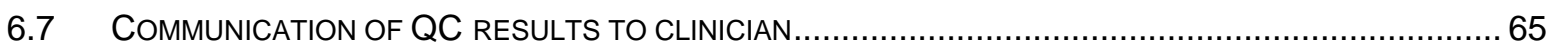

7 SUMMARY OF NCS RECOMMENDATIONS...................................................................66

APPENDIX 1. NUCLEAR DATA OF RELEVANT BETA EMITTERS …......................................68

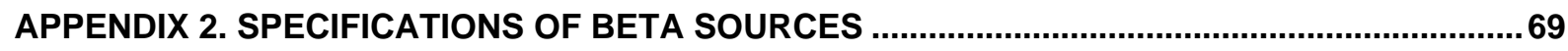

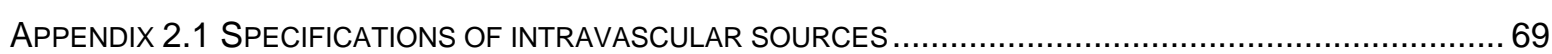

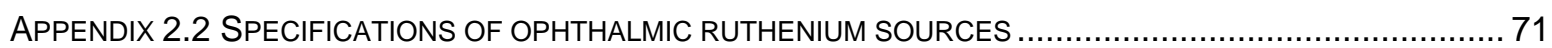

APPENDIX 2.3 DEPTH DOSE OF AMERSHAM STRONTIUM SOURCES .......................................... 72

APPENDIX 3. SCALING FROM WATER EQUIVALENT PLASTIC TO WATER ...............................73

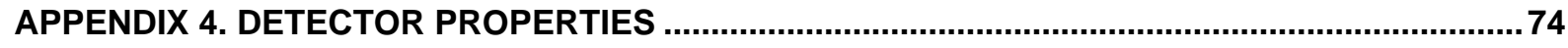

APPENDIX 5. PLASTIC SCINTILLATOR PHANTOM FOR INTRAVASCULAR SOURCES .............75

APPENDIX 6. TECHNICAL DRAWING OF FILM PHANTOM FOR INTRAVASCULAR SOURCES .76

APPENDIX 7. TECHNICAL DRAWINGS OF PHANTOMS FOR OPHTHALMIC SOURCES .............77

APPENDIX 8. SETUP FOR MEASUREMENTS ON OPHTHALMIC SOURCES...............................78

APPENDIX 9. SETUP FOR IN-WATER DIODE MEASUREMENTS ON RUTHENIUM SOURCES ...79

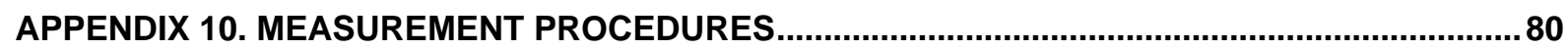

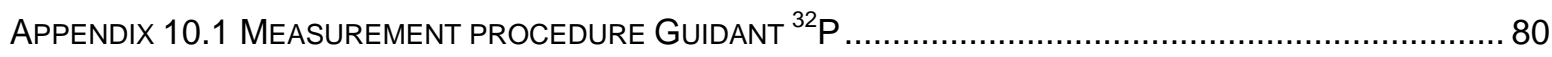

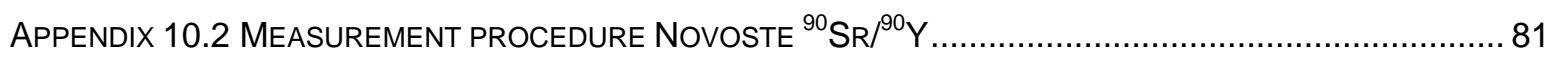

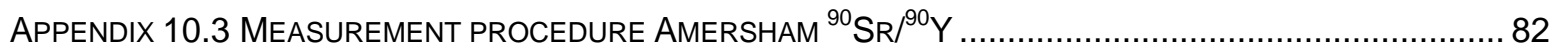

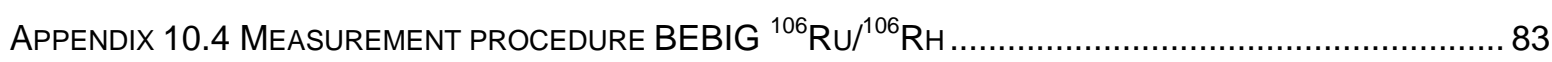

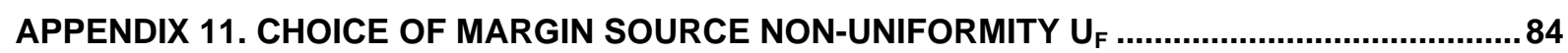

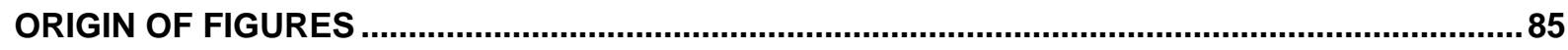

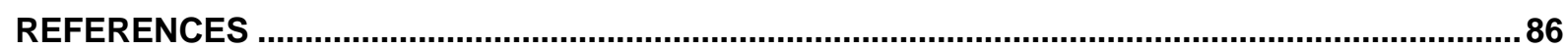


https://doi.org/10.25030/ncs-014 The NCS report has been downloaded on 26 Apr 2023 


\section{Summary}

At present, several types and subtypes of beta sources are in use in radiotherapy institutes in the Netherlands and Belgium. There is large variation in quality control - and more specifically in dosimetry - of these sources. In this report recommendations are presented on detectors and measurement procedures for the dosimetry of beta sources. In addition, recommendations are given on the quality control procedures for beta sources in general, as well as action levels for deviations in source strength and uniformity.

The recommendations on suitable detectors and measurement procedures are based on onsite measurements at 21 radiotherapy institutes in the Netherlands and Belgium. These experiments were performed with well-type ionization chambers, a plastic scintillator, a plane-parallel ionization chamber, radiochromic film and a diode. Based on this experience, parameters are proposed for the evaluation of source strength, non-uniformity and - in the case of ophthalmic sources - asymmetry.

Recommendations are presented for quality control of beta sources. On one hand, recommendations are given on the dosimetric parameters that should be determined and on the maximum deviations. On the other hand recommendations are given on, for example, the accuracy of source positioning, frequency of dosimetry and radiation safety aspects, such as measurements of leakage radiation.

With these recommendations it is expected that standardization of dosimetry and quality control of beta sources in the Netherlands and Belgium will improve and that the chance of treatment failure or emergency situations will reduce. With the availability of a primary standard for beta dosimetry at the Netherlands Measurements institute (NMi), physicists will be able to perform absolute measurements on beta sources using a calibrated detector. 


\section{Abbreviations}

$\begin{array}{ll}\text { 3D } & \text { three-dimensional } \\ \text { A } & \text { annually } \\ \text { AAPM } & \text { American Association of Physicists in Medicine } \\ \text { ASL } & \text { active source length (in mm, defined on page 14) } \\ \text { BT } & \text { brachytherapy } \\ \text { CABG } & \text { coronary arterial bypass graft } \\ \text { DGMP } & \text { Deutsche Gesellschaft fur Medizinische Physik e.V. } \\ \text { EPR } & \text { electron paramagnetic resonance } \\ \text { ESTRO } & \text { European Society for Therapeutic Radiation Oncology } \\ \text { EVA GEC } & \text { EVA: endovascular, GEC: Groupe Européen de Curietherapie } \\ \text { HDR } & \text { high dose rate } \\ \text { IAEA } & \text { International Atomic Energy Agency } \\ \text { ICRU } & \text { International Commission on Radiation Units and Measurements } \\ \text { IVBT } & \text { intravascular brachytherapy } \\ \text { IVUS } & \text { intravascular ultrasound } \\ \text { M } & \text { monthly (3M: every 3 months, etc.) } \\ \text { MRI } & \text { magnetic resonance imaging } \\ \text { NCS } & \text { Netherlands Commission on Radiation Dosimetry } \\ \text { NIST } & \text { National Institute of Standards and Technology, the US standards laboratory } \\ \text { NMi } & \text { Netherlands Measurements Institute, the Dutch standards laboratory } \\ \text { NMR } & \text { nuclear magnetic resonance } \\ \text { NSL } & \text { nominal source length (in mm, defined on page 44) } \\ \text { OD } & \text { optical density } \\ \text { P } & \text { (every) patient, every clinical application of the source } \\ \text { PDD } & \text { percentage depth dose } \\ \text { PMMA } & \text { poly(methyl methacrylate), a plastic also known as perspex or lucite } \\ \text { PPIC } & \text { plane-parallel ionization chamber } \\ \text { PS } & \text { plastic scintillator } \\ \text { PTCA } & \text { percutaneous transluminal coronary angioplasty } \\ \text { QA } & \text { quality assurance } \\ \text { QC } & \text { quality control } \\ \text { QCA } & \text { quantitative coronary angiography } \\ \text { RIL } & \text { reference isodose length } \\ \text { SD } & \text { standard deviation } \\ \text { SE } & \text { (each) source exchange } \\ \text { SS } & \text { source strength } \\ \text { TG } & \text { task group (of the AAPM) } \\ \text { TLD } & \text { thermoluminescent dosemeter } \\ \text { WIC } & \text { well-type ionization chamber } \\ & \end{array}$




\section{List of symbols}

\begin{tabular}{|c|c|c|}
\hline Symbol & Description & More information on page \\
\hline$\overline{A S L}$ & 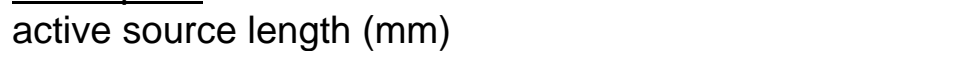 & 14 \\
\hline$\dot{D}_{a v g}$ & average absorbed dose rate (Gy/s) & 16 \\
\hline$D_{m}$ & absorbed dose to material m (Gy) & \\
\hline$\dot{D}_{\max }$ & maximum absorbed dose rate (Gy/s) & 16 (line), 17 (ophthalmic) \\
\hline$\dot{D}_{\min }$ & minimum absorbed dose rate (Gy/s) & 16 (line), 17 (ophthalmic) \\
\hline$\dot{D}_{r=0}$ & absorbed dose rate on the $z$-axis $(r=0)(G y / s)$ & 17 \\
\hline$D_{w}$ & absorbed dose to water (Gy) & \\
\hline$\dot{D}\left(r_{0}, z_{0}\right)$ & absorbed dose rate in reference point $\left(r_{0}, z_{0}\right)$ & 16 \\
\hline$\dot{D}\left(r_{0}\right)$ & average absorbed dose rate along source axis at $r=r_{0}$ & 15 \\
\hline M & margin $(\mathrm{mm})$ & 15 \\
\hline$M_{P}$ & margin for phosphorus line sources $(\mathrm{mm})$ & 15 \\
\hline$M_{S r}$ & margin for strontium line sources (mm) & 15 \\
\hline$O D$ & optical density & 35 \\
\hline$r$ & distance along the radial axis (see figure) $(\mathrm{mm})$ & 13 \\
\hline$r_{0}$ & reference position in r direction $(\mathrm{mm})$ & 13 \\
\hline$R_{50}$ & average radius of ophthalmic sources $(\mathrm{mm})$ & 16 \\
\hline$R_{c}$ & radius of curvature $(\mathrm{mm})$ & 12 \\
\hline$T_{1 / 2}$ & half-life & \\
\hline$U_{A S}$ & source asymmetry (\%) & 19 \\
\hline$U_{F}$ & source non-uniformity (flatness) (\%) & 16,17 \\
\hline$U_{\text {ICRU }}$ & source non-uniformity (ICRU definition) (\%) & 15,17 \\
\hline$z$ & distance along the $z$-axis (see figure) (mm) & 13 \\
\hline$z_{0}$ & reference position in z direction $(\mathrm{mm})$ & 13 \\
\hline
\end{tabular}


https://doi.org/10.25030/ncs-014 The NCS report has been downloaded on 26 Apr 2023 


\section{Introduction}

In many radiotherapy departments sealed beta sources are used for intravascular brachytherapy or for irradiation of eye melanoma or pterygium [1]. As for all sources of ionizing radiation used in radiotherapy, quality control (QC) of the beta sources is the responsibility of the clinical or medical physicist. An important aspect of this quality control is (independent) verification of the dosimetric properties of the source, such as strength and uniformity. In comparison with gamma sources, beta sources have a short range (typically about $1 \mathrm{~cm}$ in water) and a steep dose gradient. This gradient is also present in the detection volume and small changes in source or detector positioning may give rise to large deviations (for example a $0.1 \mathrm{~mm}$ change in position may cause a $10 \%$ difference in absorbed dose). For the calibration of most beta sources no standardized techniques are available, and it is difficult to find calibrated detectors for dosimetry of these sources. Until recently, only one primary standard for clinical beta sources was available wordwide (at NIST in the USA).

Source strength and depth dose measurements on beta sources have been described in the literature and one of the manufacturers provides a calibrated detector for verification of source strength. Several organizations, such as the ICRU [2] and the IAEA [3], have published an overview of detectors that may be used. The dosimetric properties of interest have been described by Task Group 60 of the AAPM [4] and the DGMP [5], as well as the ICRU and the IAEA $[2,3]$. For the QC of brachytherapy sources in general, recommendations are available in the report of Task Group 56 of the AAPM [6] and in NCS Report 13 [7]. This latter report gives recommendations on the $\mathrm{QC}$ of beta sources, but not on detectors for beta dosimetry. Only the EVA GEC ESTRO working group [8] gives such a recommendation for beta line sources. Guidelines were always written with gamma sources in mind and, therefore, no specific QC for beta sources has been described.

In this report tests and a selection of detectors for the dosimetry of beta sources are presented. These detectors were evaluated employing several criteria, both physical (e.g. sensitivity, stability) and practical (e.g. ease of use, cost). Recommendations are given on suitable detectors and on the parameters that should be determined. A compilation of the measurement procedures used is given in Appendix 10. Based on our experience with sources used in clinical practice, tolerances are set for deviations in source strength, for source non-uniformity and asymmetry. In addition to these dosimetric aspects, a set of recommendations for QC in general is presented, divided into radiation safety aspects and non-dosimetric physical parameters.

Parallel to the recommendations presented in this report a primary standard for beta sources has become available at the Netherlands Measurements institute (NMi). At this facility detectors can be calibrated for independent measurements on beta sources. Radioactive sources with a long half-life such as strontium eye applicators can also be sent to the NMi for calibration. 


\subsection{Purpose}

This NCS report gives recommendations on quality control procedures for beta sources. Recommendations are also given on suitable detectors used for dosimetry of beta sources. Action levels are set for deviations in strength and for maximum source non-uniformity and asymmetry. In addition to these dosimetric aspects, recommendations are given on the frequency of dosimetry and on other quality control procedures, such as the accuracy of source positioning and background radiation measurements.

\subsection{Scope}

In the remainder of this chapter clinical indications for brachytherapy with beta sources as well as irradiation techniques used are described. An overview of the radioactive beta sources currently being used in the Netherlands and Belgium is given in Chapter 2.

Measurement techniques are introduced in Chapter 3. This chapter describes the coordinate systems for beta sources, detectors for dosimetry of beta sources and corrections that need to be taken into account in measurements. The current status of dosimetry of beta sources in the Netherlands and Belgium is described in Chapter 4 . To be able to give recommendations on detectors for dosimetry of beta sources, measurements were performed with a number of detectors. Materials and methods of these measurements are given in Chapter 5, together with the results and an evaluation. Recommendations on detectors, measurement concepts and reference points for dosimetry of beta sources in clinical practice are given in Chapter 6 . The same chapter provides recommendations on quality control procedures for beta sources. The scope and frequency of measurements is described as well as action levels for deviations in source strength and uniformity. Other physical parameters and safety aspects that should be taken into account are also described. Chapter 7 contains a summary of the recommendations.

\subsection{Interpretation of the recommendations}

This report contains recommendations for quality control procedures, frequency of measurements and tolerances in source strength and uniformity. Part is given as a minimum requirement, part is optional. Each user, however, has his or her own responsibility in implementing these recommendations. The recommendations on detectors only apply to detectors that were investigated and that were found to be suitable for source strength or uniformity measurements. New detectors or detectors that were not investigated may also be suitable, provided they fulfil the same criteria. 


\subsection{Treatment indications}

\subsubsection{In-stent restenosis}

Narrowing of coronary arteries is one of the leading causes of death in the western world. Nowadays more than one million PTCAs* are performed annually throughout the world. In the past few years intravascular brachytherapy, with the purpose of reducing restenosis following PTCA, has been a rapidly expanding application of beta sources. Restenosis occurs in about $30-50 \%$ of the PTCAs $[9,10]$, depending on parameters such as length of the lesion.

Restenosis results from proliferation of the intima and constrictive remodelling of the injured artery. The use of a stent to prevent remodelling of an artery reduces the restenosis rate [11], but in-stent restenosis is the primary indication for intravascular brachytherapy. Absorbed doses in the range of 15-25 Gy at a radial distance of $2 \mathrm{~mm}$ from the source axis are used in intravascular brachytherapy [12-15]. The in-stent restenosis is reported to reduce with about $50 \%$, resulting in angiographic restenosis rates between $8-26 \%[14,16-18]$, depending on the lesion characteristics.

In the first years of intravascular brachytherapy ${ }^{192}$ Ir played an important role $[19,20]$.

Minimizing patient dose outside the target area and minimizing the dose to personnel were important reasons for the introduction of beta sources [21]. ${ }^{32} \mathrm{P}$ and ${ }^{90} \mathrm{Sr} /{ }^{90} \mathrm{Y}$ have taken over the leading role of ${ }^{192} \operatorname{Ir}$ [16-18]. In 2001 about 800-900 patients were treated with intravascular brachytherapy in the Netherlands and Belgium. There are, however, new developments to reduce restenosis. With the introduction of drug eluting stents [22,23] a new era seems to have arrived, in which the role of intravascular brachytherapy might be limited to that of a complementary therapy. Intravascular brachytherapy is expected to remain an option for patients who are not eligible for placement of a new stent or CABG (Coronary Arterial Bypass Graft). Other trials have been initiated to investigate the role of vascular brachytherapy for treating peripheral artery disease and arterialvenous dialysis graft stenosis.

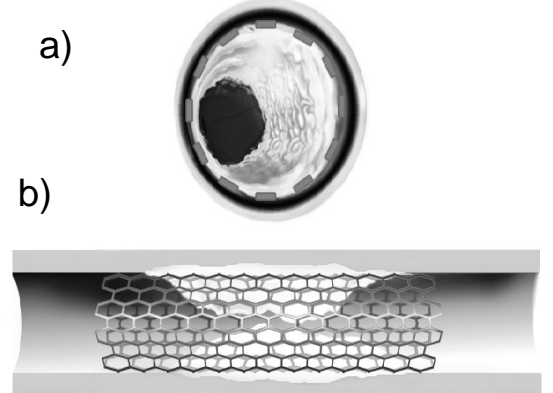

Figure 1-1 Example of in-stent restenosis, the primary indication for intravascular brachytherapy.

a) cross-section, showing vessel, restenosis and stent

b) longitudinal section of the vessel

\subsubsection{Pterygium}

The oldest application of beta sources in radiotherapy dates from the fifties, when strontium eye applicators were used for the treatment of pterygium [24]. Pterygium is a triangular growth of fibrovascular tissue of the bulbar conjunctiva that invades the cornea and results in visual disturbances (Figure 1-2). Pterygium is a benign disease, treated by excision of the affected part of the sclera. Postoperative irradiation of this area reduces the recurrence from $20-40 \%$ to less than 5\% [25]. In the Netherlands and Belgium this indication most frequently occurs in patients who have been exposed to a large amount of UV radiation (often during presence close to the equator). During treatment, the eye is irradiated in several (weekly) fractions. Most institutes use 3x10 Gy surface dose fractions, based on the experience of van

\footnotetext{
PTCA: Percutaneous (through the skin) Transluminal (through the lumen) Coronary (pertaining to the heart) Angioplasty (repair of a blood vessel), also known as dottering.
} 
den Brenk in the sixties [26]. In the Netherlands and Belgium, every year about 400 patients are irradiated after surgery ${ }^{*}$. Strontium sources with a concave or flat surface are used for the treatment. Treatment times vary from 20 seconds to 5 minutes, depending on the dose rate. The use of ${ }^{90} \mathrm{Sr} /{ }^{90} \mathrm{Y}$ is particularly attractive because the eye lens, with the edge 2-3 mm beneath the surface, is very sensitive to radiation. Cataract is the most important complication of the treatment.

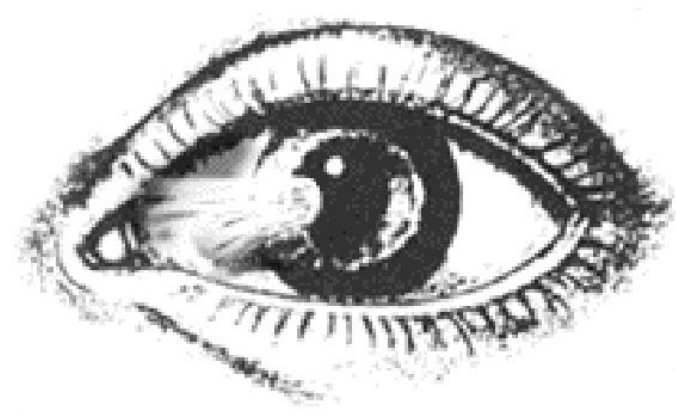

Figure 1-2 Example of pterygium.

\subsubsection{Melanoma of the eye}

A more limited application of beta sources is the treatment of choroidal melanoma [27] and retinoblastoma [28]. These malignant diseases are treated with concave ruthenium plaques that can be stitched to the eye. At low dose rate the tumor is irradiated during a period varying from two up to fourteen days, depending on the activity of the source. The highenergy beta emitter ruthenium is used because tumor thickness can be up to $10 \mathrm{~mm}$. Because the largest part of the absorbed dose is deposited in the first $10 \mathrm{~mm}$ from the source surface, and most tumors are situated at the posterior side of the eyeball (Figure 1-3), the absorbed dose to the lens usually is limited. The prescribed dose for treating choroidal melanoma is typically $80-120$ Gy at the apex of the tumor. This is, however, limited by the surface dose that should not exceed 1000 Gy [29]. In the literature the use of strontium sources for treatment of eye melanoma has also been reported [30]. Of all eye melanoma, uveal melanoma has the highest (yearly) incidence with six out of one million people. Over a period of 15 years the overall survival rate is estimated to be about $48 \%$ and the cumulative local treatment failure is 37\% [31]. Ciliar body melanoma (near the iris) and melanoma close to the optical nerve require use of applicators with a special shape.

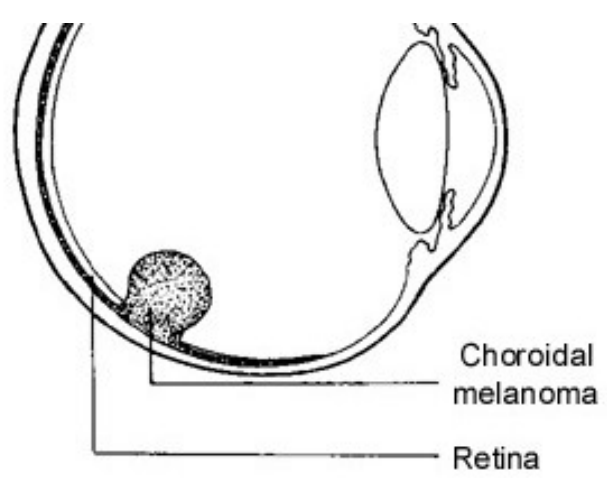

Figure 1-3 Example of a choroidal melanoma.

\footnotetext{
Numbers of patients used in this section are based on the results of the questionnaire described in Chapter 4.
} 


\subsection{Irradiation techniques}

Whilst all ophthalmic sources are placed directly on the target area (using tweezers or a handle), sources for intravascular brachytherapy are delivered through a catheter in the groin, via other blood vessels and into the coronary artery. This is done by afterloading. In intravascular brachytherapy two types of devices were available commercially at the time of writing: an automatic afterloading device (Figure 1-4) and a handheld device (Figure 1-5). Usually delivery of the source is performed after PTCA and is based on the position and size of the PTCA balloon taking into account appropriate margins [8]. Source positioning is guided by markers in the catheter that are visible on angiography. The Novoste (Norcross, GA, USA) Beta-Cath ${ }^{\mathrm{TM}}$ 3.5F system brings up a dummy wire simultaneously with the catheter. After removal of the dummy wire the source is sent out. The older $5 \mathrm{~F}$ system has a separate dummy afterloading device. The Guidant (Houston, TX, USA) GALILEO тM Intravascular Radiotherapy System sends out the dummy wire after the catheter has been positioned correctly. The active wire position is defined by stepping the dummy wire to the required position after which the active source can be delivered.

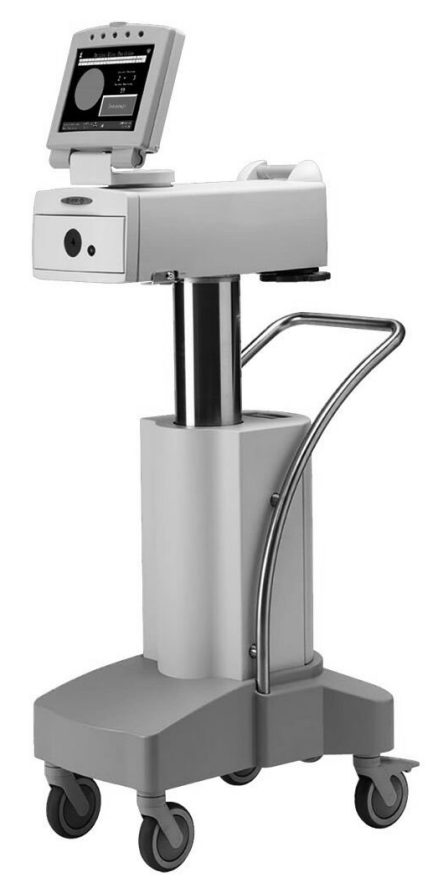

Figure 1-4 The Guidant automatic afterloader. Source delivery takes place with a source wire that is driven by the source delivery unit.

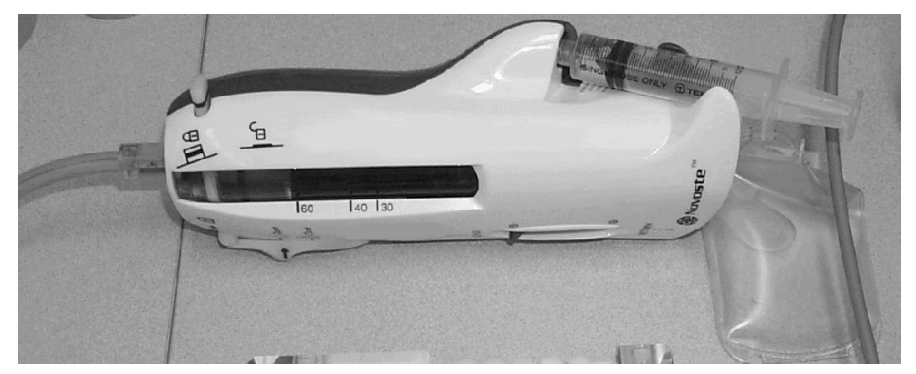

Figure 1-5 The Novoste handheld afterloader. Source delivery takes place hydraulically. 


\section{Sealed beta sources}

In this chapter sealed radioactive beta sources are described for the three application areas : intravascular brachytherapy, ophthalmic strontium and ophthalmic ruthenium applications. Non-commercial, coated balloon and radioactive stent applications do not fall within the scope of this report. An overview of the radioisotopes used, their energies and decay times are given in Appendix 1. Measured depth dose curves for intravascular and ophthalmic strontium sources are given in Appendix 2.

\subsection{Intravascular brachytherapy}

Novoste and Guidant are manufacturers of commercial intravascular brachytherapy devices presently available in the Netherlands and Belgium. Both devices contain line sources.

The Novoste source consists of a source train with 12,16 or 24 seeds of $2.5 \mathrm{~mm}$ length each, resulting in 30, 40 and $60 \mathrm{~mm}$ long trains (see Figure 2-1). The radioactive component of the seeds is ${ }^{90} \mathrm{Sr} /{ }^{90} \mathrm{Y}$. The strontium is embedded in a stainless steel encapsulation. Two versions of the Novoste sources are in use in the Netherlands and Belgium: the $5 \mathrm{~F}^{*}$ and $3.5 \mathrm{~F}$ sources. While the seeds of the $5 \mathrm{~F}$ system can move with respect to each other, the seeds of the 3.5F system are kept together by a surrounding jacket, made of stainless steel. This jacket has a coil-like shape, but fully covers the seeds. Both types of sources are transferred by a hydraulic system that is driven by manual pressure on a syringe. The diameter of the seeds is $0.38 \mathrm{~mm}$ for the $3.5 \mathrm{~F}$ sources and $0.64 \mathrm{~mm}$ for the $5 \mathrm{~F}$ sources.

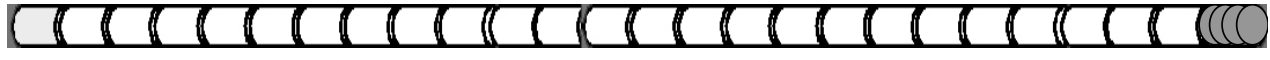

Figure 2-1 Schematic view of the $5 F^{90} \mathrm{Sr}^{90} \mathrm{Y}$ Novoste source (24 seeds of $2.5 \mathrm{~mm}=60 \mathrm{~mm}$ source, left and right of the source train is a radiopaque end marker).

Guidant uses a $20 \mathrm{~mm}$ stepping source that has ${ }^{32} \mathrm{P}$ as the radioactive component (see Figure 2-2). Larger source lengths of $40 \mathrm{~mm}$ and $60 \mathrm{~mm}$ can be achieved by stepping. The source is embedded in a NiTi encapsulation and mounted on a NiTi drive wire. The outer diameter of the NiTi tube is $0.46 \mathrm{~mm}$.
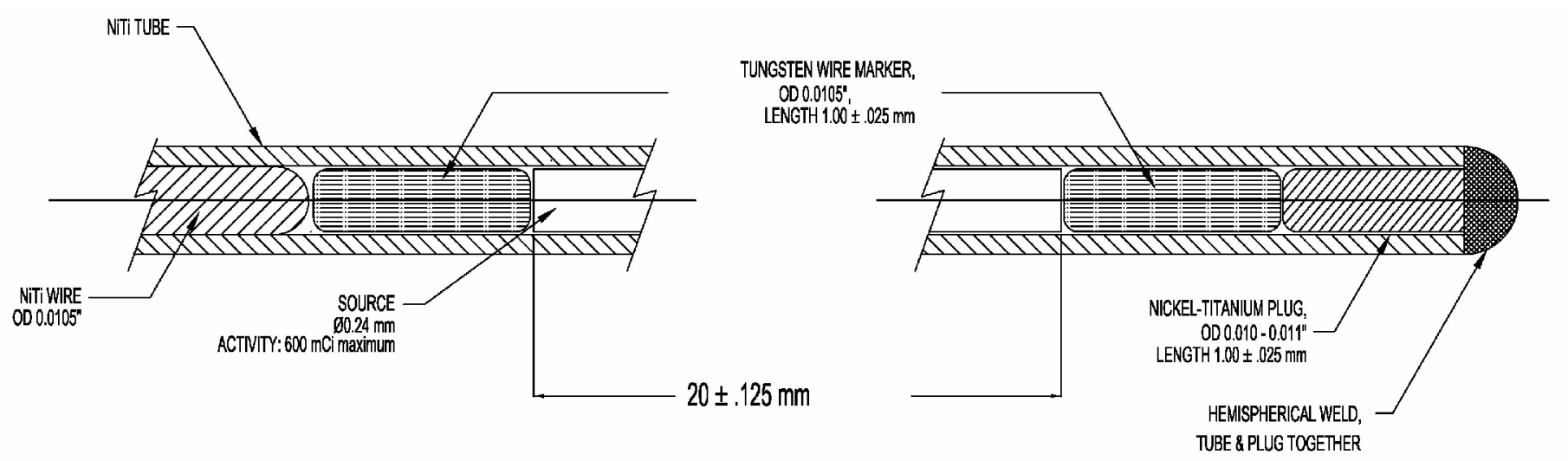

Figure 2-2 Schematic view of the $20 \mathrm{~mm}^{32} \mathrm{P}$ Guidant source.

Other relevant specifications of both the ${ }^{90} \mathrm{Sr} /{ }^{90} \mathrm{Y}$ and ${ }^{32} \mathrm{P}$ sources are summarized in Appendix 2.1.

\footnotetext{
* French $(\mathrm{F})$ is defined as the circumference of the catheter in $\mathrm{mm}$. A 3.5F catheter therefore has a diameter of $3.5 / \pi=1.11 \mathrm{~mm}$, a $5 \mathrm{~F}$ catheter of $5 / \pi=1.59 \mathrm{~mm}$.
} 


\subsection{Ophthalmic strontium applications}

Although several manufacturers have produced strontium applicators, all strontium applicators currently used in the Netherlands and Belgium were manufactured by Amersham Health (Buckinghamshire, UK). Amersham has now stopped manufacturing these sources. Two views of the planar SIA.20 source are shown in Figure 2-3. With a $9 \mathrm{~mm}$ active diameter the source usually does not need to be moved during irradiation. The radioactive strontium90 is embedded in silver and sealed with a stainless steel window that also acts as a filter to attenuate low-energy beta radiation emitted by strontium- 90 .
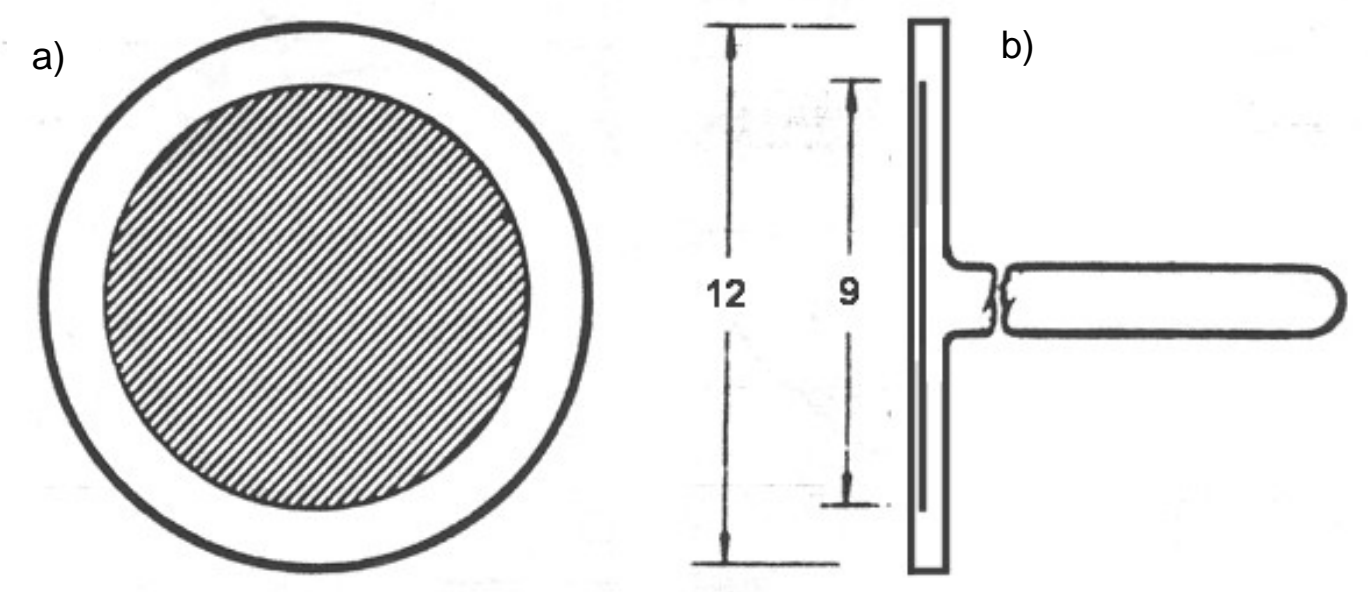

Figure 2-3 Schematic configuration of the planar SIA.20 strontium-90 source (edited from figure in Amersham catalogue). The active diameter equals $9 \mathrm{~mm}(12 \mathrm{~mm}$ total). The applicator has a thick backing and a stainless steel filter of $0.05 \mathrm{~mm}$. a) front view; b) side view. The side view shows the handle of the source.

Figure 2-4 shows the SIA.6 applicator, which is the most common concave source.

Theradius of curvature of $10 \mathrm{~mm}$ is chosen because it matches the shape of the eye.

a)

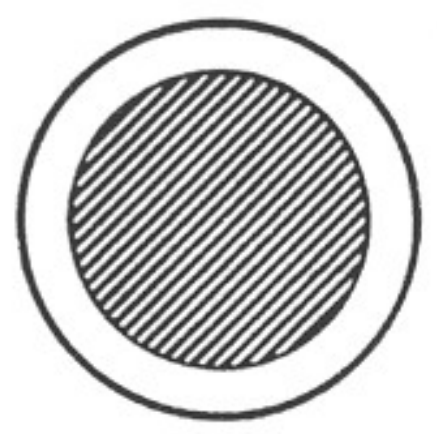

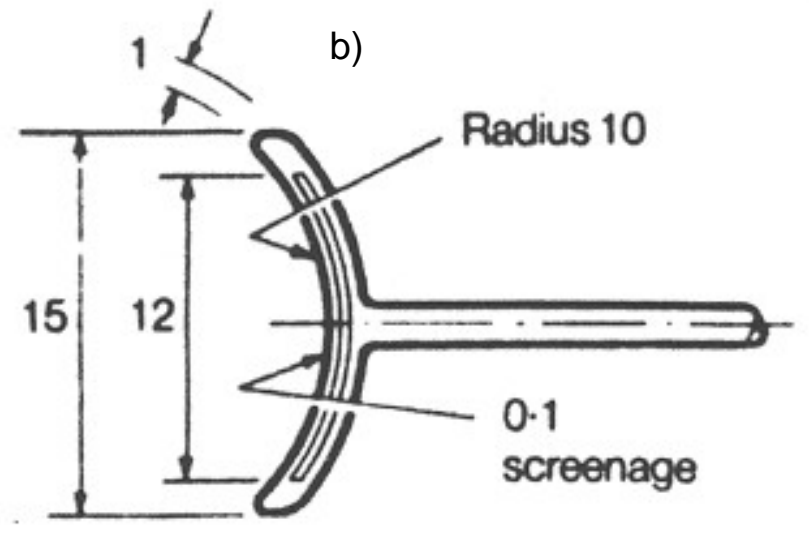

Figure 2-4 Configuration of the concave SIA.6 strontium-90 source. The active diameter equals $12 \mathrm{~mm}$ (15 mm total). The applicator is $1 \mathrm{~mm}$ thick and has a stainless steel filter of $0.1 \mathrm{~mm}$. The radius of curvature is $10 \mathrm{~mm}$. a) front view; b) side view. The side view shows the handle of the source. 


\subsection{Ophthalmic ruthenium applications}

At present, BEBIG (BEBIG Isotopen- und Medizintechnik GmbH, Berlin, Germany) is the only manufacturer of ruthenium plaques for eye irradiations. The configuration for ophthalmic plaques is shown in Figure 2-5. The ruthenium applicators are designed to have the same curvature as the eye. There are two groups of ruthenium sources: full size symmetrical sources and asymmetrical sources that have cutouts for the optical nerve (e.g. type COB) or iris (e.g. CIB), see Appendix 2.2. All plaques are made of silver, with ruthenium-106 as the radioactive component. The types in use in the Netherlands and Belgium all have a radius of of $12 \mathrm{~mm}$. Source diameter ranges from 11.6 to $20.2 \mathrm{~mm}$ enabling irradiation of tumors of different size.

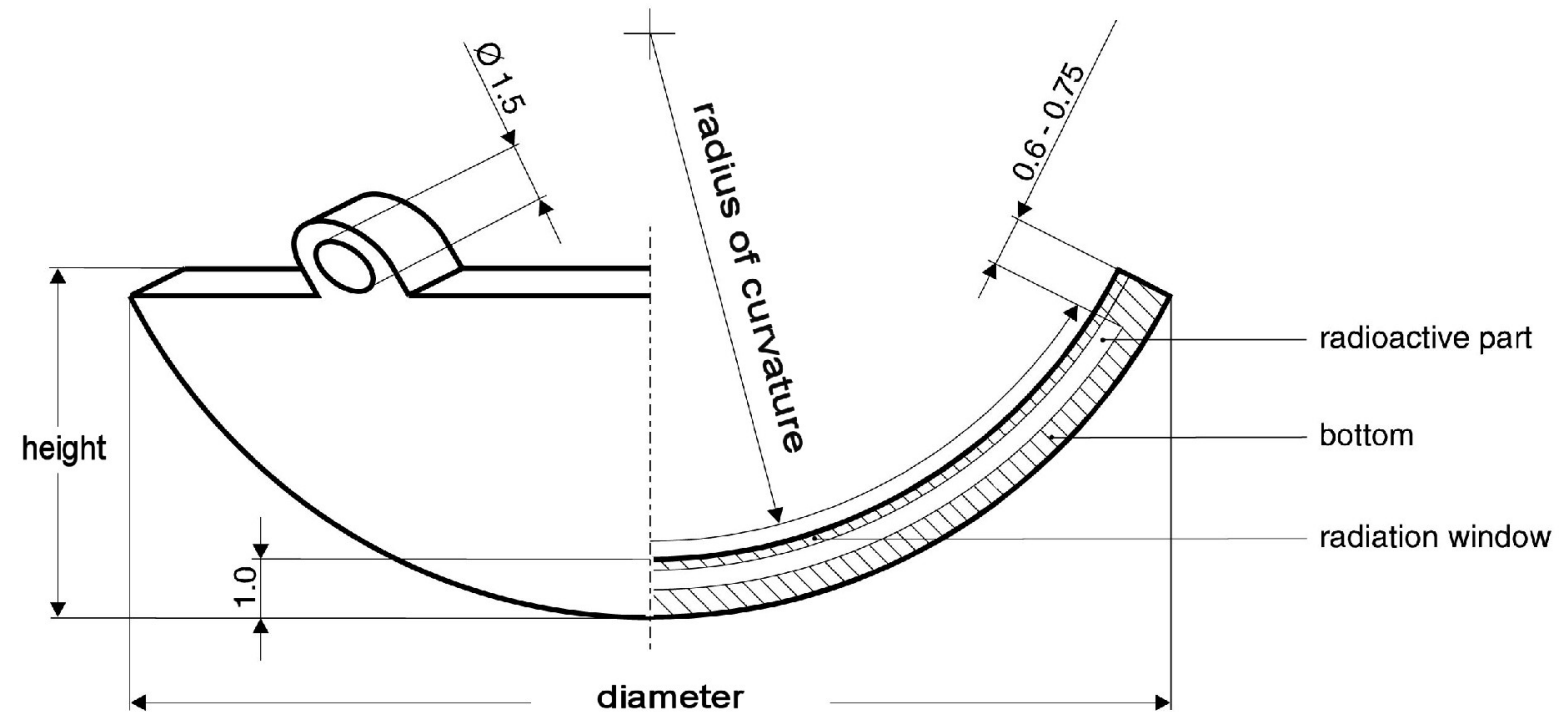

Figure 2-5 Configuration of ruthenium-106 plaques. All sources in use in the Netherlands and Belgium have a radius of curvature of $12 \mathrm{~mm}$. Both diameter and height can vary. The applicator is $1 \mathrm{~mm}$ thick and has a silver radiation window of $0.1 \mathrm{~mm}$.

\subsection{Source strength specification by manufacturer}

In Table 2-1 specifications of the source strengths provided by several manufacturers are given together with the uncertainty involved in source calibration. BEBIG introduced a new standard in 2002. Uncertainties of both old and new standards are specified. Guidant is the only manufacturer that actually specifies activity and provides a conversion factor for the absorbed dose to water. Other manufacturers specify in a reference point in water.

Table 2-1 Overview of source strength specification by manufacturers.

\begin{tabular}{|l|l|l|}
\hline Manufacturer & Specified quantity & $\begin{array}{l}\text { Standard uncertainty } \\
\text { (expanded, 2 } \sigma \text { ) }\end{array}$ \\
\hline Guidant & Activity $(\mathrm{GBq})$ & $5.4 \%$ \\
\hline Novoste & $\begin{array}{l}\text { Reference absorbed dose rate in cGy/s at 2 } \\
\text { mm in water per GBq }\end{array}$ & $16 \%$ \\
\hline Amersham & Dose rate $(\mathrm{Gy} / \mathrm{s}$ ) at $2 \mathrm{~mm}$ in water & $20 \%$ \\
\hline $\begin{array}{l}\mathrm{BEBIG} \\
\text { (old standard) }\end{array}$ & $\begin{array}{l}\text { Surface dose rate }\left(\mathrm{rads} / \mathrm{s} \text { ) at } 7 \mathrm{mg} / \mathrm{cm}^{2}\right. \\
\text { (calculated for 0 } \mathrm{mm} \text { in water) }\end{array}$ & $20 \%(3 \sigma)$ \\
\hline $\begin{array}{l}\text { BEBIG } \\
\text { (current standard) }\end{array}$ & $\begin{array}{l}\text { Dose rate }(\mathrm{mGy} / \mathrm{min} \text { ) at } 0.7 \mathrm{~mm} \text { in water } \\
\text { (calculated for 0 and 2 } \mathrm{mm} \text { in water) }\end{array}$ & $20 \%$ \\
\hline
\end{tabular}




\section{Measurement techniques}

This chapter contains an overview of different techniques and methodologies required for the dosimetry of beta sources, such as coordinate systems and reference points (Section 3.1), dosimetric parameters (Section 3.2), possible detectors (Section 3.3), and measurement corrections (Section 3.4). It should be noted that this chapter only provides background information and that the recommendations of the NCS can be found in Chapter 6. Criteria for selection of detectors and a first selection based on these criteria are given at the end of the present chapter.

\subsection{Source coordinate systems and reference points}

The $r$ and $z$-axis of line, planar and concave sources are defined in Figure 3-1. In this report reference points are defined (in $\mathrm{mm}$ ) in water at $\left(r_{0}, z_{0}\right)=(2,0)$ for line sources and at $\left(r_{0}, z_{0}\right)=$ $(0,2)$ for ophthalmic sources. As depicted in Figure 3-1a, for line sources the origin is defined at the center of the source. The z-axis is the source axis and the r-axis is perpendicular to the source axis. The $z$-axis is also the source axis of the concave and planar sources. In this case the origin is located at the surface of the source (in all figures the origin is indicated by a black dot). For ophthalmic sources the reference points are all positioned on the central source axis. The source strength should be expressed as the absorbed dose rate to water at the reference point $\dot{D}\left(r_{0}, z_{0}\right)$ (ophthalmic sources) or at a reference distance $\dot{D}\left(r_{0}\right)$ (line sources).

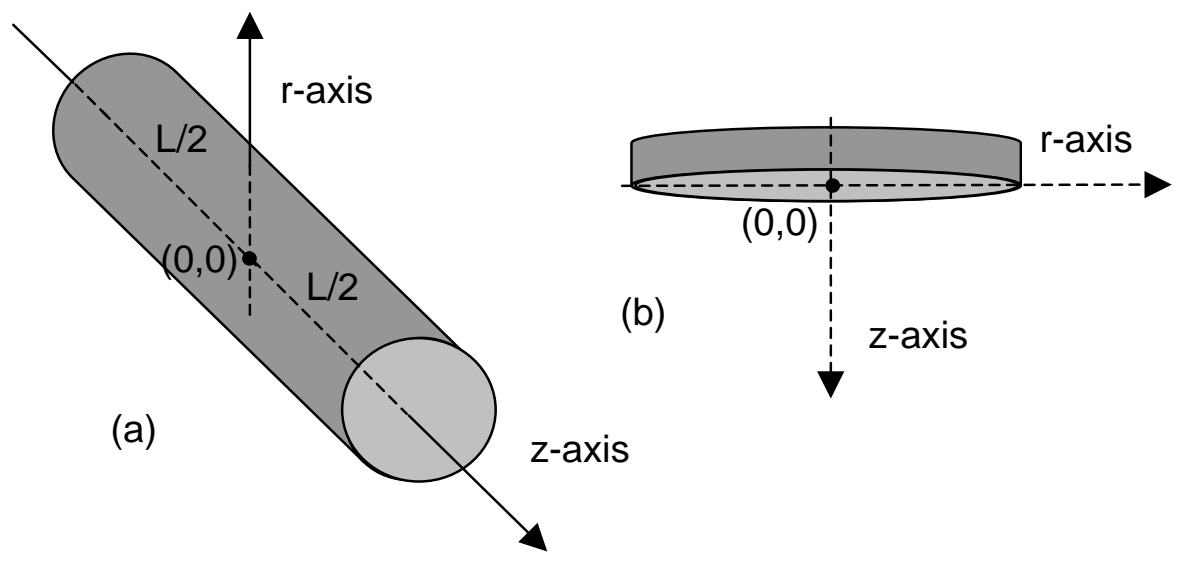

(c)

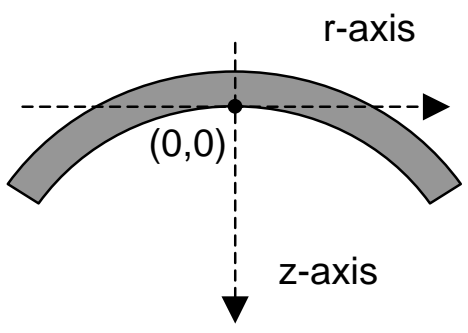

Figure 3-1 Coordinate systems for a) intravascular line sources, b) planar ophthalmic sources and c) concave ophthalmic sources.

\subsection{NCS definitions of dosimetric parameters for sealed beta sources}

In the following subsections parameters are introduced for the quantification of source strength, non-uniformity and asymmetry. The NCS proposes to use mutually related concepts for source strength and source non-uniformity, in which the non-uniformity describes the maximum deviation from the source strength that can be expected within the area of interest for both intravascular and ophthalmic sources. For line sources, the source strength is defined as the average absorbed dose along the source axis and the nonuniformity is defined as the largest possible deviation from that value. For ophthalmic sources, the source strength is defined at the reference point and the source non-uniformity is the largest possible deviation within the area of interest. 


\subsubsection{Intravascular brachytherapy sources}

\section{Active Source Length (ASL)}

\section{Overview of literature}

According to the EVA GEC ESTRO working group [8] the Active Source Length is defined as follows:

"The ASL is defined as the length of the radioactive source(s) or source train or the length of the active dwell positions of a stepping source."

In Figure 3-2 this definition is shown as a longitudinal source profile.

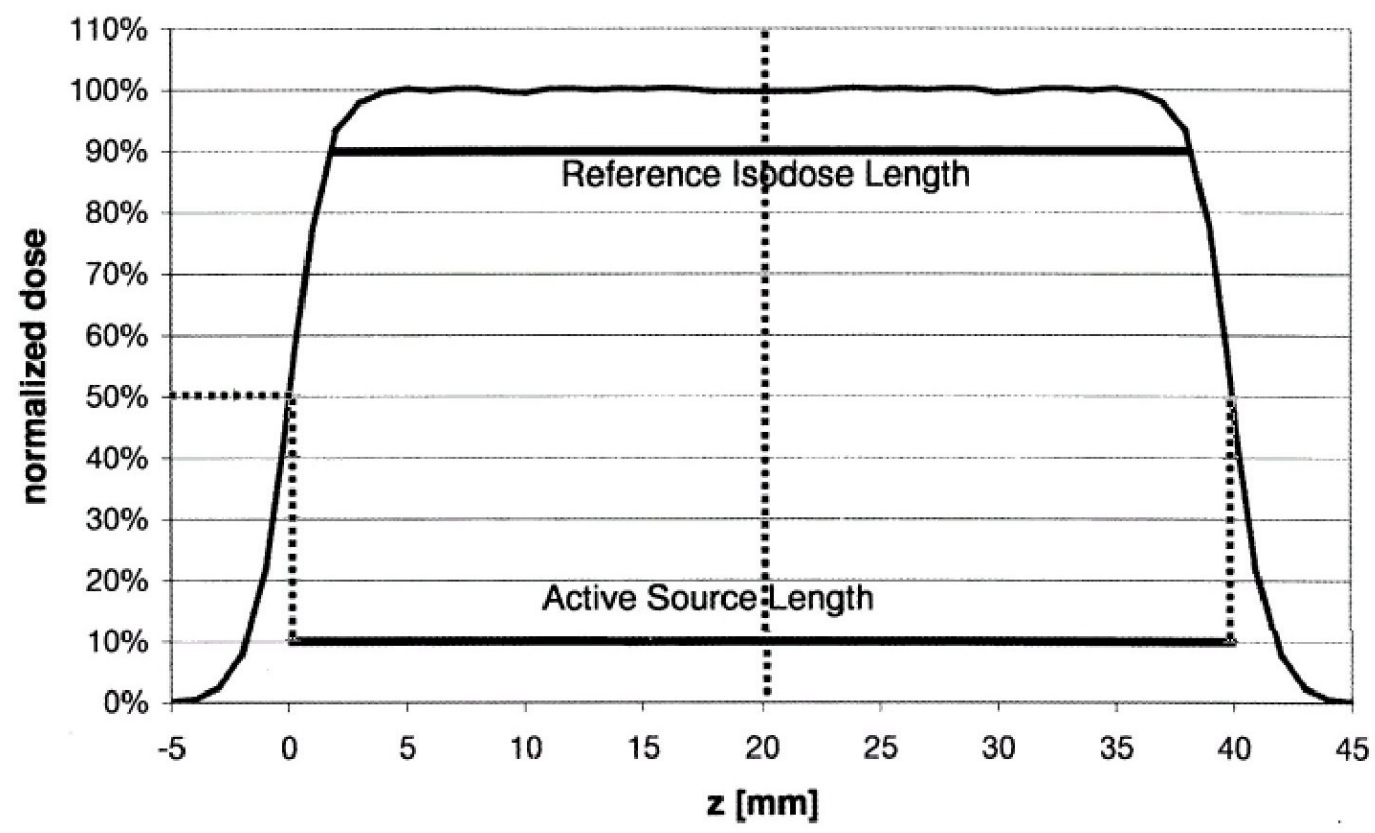

Figure 3-2 Active source length as defined in the EVA GEC ESTRO document.

\section{NCS definition}

The EVA GEC ESTRO document provides a suitable basis for evaluation of the source length. Because the $100 \%$ level of the ASL has not been defined explicitly in this document, the NCS recommends to use the maximum absorbed dose at $r=2 \mathrm{~mm}$ as the $100 \%$ level. The ASL is defined as the distance between the $50 \%$ points on the longitudinal dose distribution. (Other $100 \%$ definitions would only give small differences in the ASL value, due to the steep penumbra of beta sources. A $100 \%$ definition based on the ASL minus a margin - as described on the next page - is not possible, because the source profile needs to be normalized first.)

\section{Source strength}

\section{Overview of literature}

In its report on intravascular brachytherapy [4] the AAPM TG (Task Group) 60 describes how the source strength and dose distribution of intravascular beta sources should be specified: "The output of all commercial systems for coronary applications should be specified in terms of dose rate in water at a radial distance of $2 \mathrm{~mm}$ from the center of the source."

"The penetrating ability of all commercial systems should be specified in terms of the radial dose function normalized at a radial distance of $2 \mathrm{~mm}$. The radial dose function should cover at least a range of radial distances from 0.5 to $10 \mathrm{~mm}$ (or $\mathrm{R}_{90}, 90 \%$ of the electron range for beta emitters) at $0.5 \mathrm{~mm}$ intervals." 
These recommendations form the basis for all other recommendations on source strength of beta line sources $[2,5,8]$. In practice, the source strength in terms of dose rate is either determined directly (with a point detector) or indirectly (with a well-type ionization chamber). In the case of an indirect measurement, Monte Carlo simulations often are used for calculation of the source strength at the reference point.

For intravascular applications in peripheral arteries the reference point is defined at $r=5 \mathrm{~mm}$ in water instead of $2 \mathrm{~mm}$ [5]. Currently, no commercial beta sources are in use for this application.

\section{NCS definition}

For three reasons, the NCS proposes an alternative to the AAPM concept of source strength using the average of the absorbed dose rate along the source axis. Firstly, the two manufacturers of beta line sources (Guidant and Novoste) perform their calibrations with detectors that evaluate the entire source length. Secondly, from a clinical point of view, the average absorbed dose rate along the source is more relevant than the absorbed dose rate in a single point. Thirdly, only a fraction of the source length contributes to the dose rate at $\left(r_{0}, z_{0}\right)=(2,0)$ due to the limited range of betas. Therefore the NCS recommends to define the source strength as the average absorbed dose rate $\dot{D}\left(r_{0}\right)$ at $r_{0}=2 \mathrm{~mm}$ along the active source length minus a margin $M$ at both ends of the source. $M_{P}=2.5 \mathrm{~mm}$ for ${ }^{32} \mathrm{P}$ sources and $M_{S r}=3.0 \mathrm{~mm}$ for ${ }^{90} \mathrm{Sr}{ }^{90} \mathrm{Y}$ sources. Simulated source profiles are given in Appendix 11. It is noted that with these margins, the source strengths of the ${ }^{32} \mathrm{P}$ and ${ }^{90} \mathrm{Sr} /{ }^{90} \mathrm{Y}$ line sources are affected by less than $0.5 \%$ by the dose fall-off near the ends of the sources.

\section{Source non-uniformity}

\section{Overview of literature}

The AAPM TG 60 [4] recommends to evaluate the uniformity of a source along the source axis and in the azimuthal direction according to the following requirements :

"Uniformity of the dose delivered at points along the source-axis should be better than $\pm 10 \%$ (range of values from minimum to maximum in the centered two-thirds of the treatment length of at least $3 \mathrm{~cm}$ ) at a radial distance of $2 \mathrm{~mm}$ from the source axis."

"Uniformity of a dose at the points in a circle of $2 \mathrm{~mm}$ radius centered on the course axis should also be within $\pm 10 \%$ in the centered two-thirds of a minimum treatment length of 3 cm."

In its draft report the ICRU [2] advises to apply a different concept of source non-uniformity. In this concept the difference between minimum and maximum is evaluated, instead of the deviation from a reference value. Although the ICRU uses $U$ for non-uniformity, $U_{\text {ICRU }}$ is used throughout this document for clarity. The following formula is used:

$U_{\text {ICRU }}=\left(\dot{D}_{\text {max }}-\dot{D}_{\text {min }}\right) / \dot{D}_{\text {avg }} \times 100 \%$

in which:

$$
\begin{aligned}
& U_{I C R U}=\text { source non-uniformity }(\%) \\
& \dot{D}_{\max }=\text { maximum absorbed dose rate } \\
& \dot{D}_{\min }=\text { minimum absorbed dose rate } \\
& \dot{D}_{\text {avg }}=\text { average absorbed dose rate }
\end{aligned}
$$

This concept applies to the same $2 / 3$ of the source length as described by the AAPM TG 60 . The ICRU recommends a maximum non-uniformity of $20 \%$. In contrast to the AAPM TG 60 recommendation, this concept gives a clear normalization using the average absorbed dose. 


\section{NCS definition}

The NCS gives preference to a non-uniformity concept that evaluates the maximum deviation from the source strength. Therefore an approach similar to that of TG 60 is followed, except that it is linked to the concept of source strength described in the previous section. The AAPM recommendation to evaluate the source non-uniformity over $2 / 3$ of the source length is not considered appropriate for beta sources, because this may result in unacceptably large margins of $10 \mathrm{~mm}$ at both ends of a $60 \mathrm{~mm}$ source. The NCS recommends to evaluate the source non-uniformity over the active source length minus a margin $M$ at both ends of the source. $M_{P}=2.5 \mathrm{~mm}$ for ${ }^{32} \mathrm{P}$ sources and $M_{S r}=3.0 \mathrm{~mm}$ for ${ }^{90} \mathrm{Sr} /{ }^{90} \mathrm{Y}$ sources. The choice for these margins is discussed in Appendix 11 and is based on data of clinical sources as presented in Chapter 5. It is noted that the dose rates of the ${ }^{32} \mathrm{P}$ and ${ }^{90} \mathrm{Sr}{ }^{90} \mathrm{Y}$ line sources are at $96-97 \%$ of the maximum value at a distance $M$ from the $50 \%$ point if the sources are assumed to be perfectly uniform [32]. The portion (ASL-2M) of the source length that is determined by these margins is used for the definition of the average absorbed dose of the source. The NCS proposes to use the symbol $U_{F}$ (with $F$ for flatness) for non-uniformity defined as:

$U_{F}=\max \left(\left|\dot{D}_{\min }-\dot{D}_{\text {avg }}\right|,\left|\dot{D}_{\max }-\dot{D}_{\text {avg }}\right|\right) / \dot{D}_{\text {avg }} \times 100 \%$

in which:

$$
\begin{aligned}
& U_{F}=\text { source non-uniformity (\%) } \\
& \dot{D}_{\max }=\text { maximum (relative) absorbed dose rate within ASL-2M } \\
& \dot{D}_{\min }=\text { minimum absorbed dose rate within ASL-2M } \\
& \dot{D}_{\text {avg }}=\text { average absorbed dose rate within ASL-2M }
\end{aligned}
$$

\subsubsection{Ophthalmic sources}

\section{Average radius $(R 50)$}

The average radius $\left(R_{50}\right)$ is defined as the mean radius of the $50 \%$ isodose contour of a dose distribution in which $100 \%$ is given by the maximum absorbed dose [2]. A precise description of how this radius can be calculated is given in the section on source non-uniformity (see below).

\section{Source strength}

\section{Overview of literature}

In the (draft) ICRU report [2] the source strength of all planar and concave sources is defined as the dose rate at $(r, z)=(0,1) \mathrm{mm}$. The IAEA [3] recommends using the same point but recognizes that performing measurements at these short distances is a difficult task.

\section{NCS definition}

The NCS supports the IAEA opinion that measuring the absorbed dose at a distance of 1 $\mathrm{mm}$ is a difficult task. Curvature of concave sources and minimum thickness of spacers limit the possibilities of performing measurements at $1 \mathrm{~mm}$, even if detector dimensions are sufficiently small. The NCS therefore recommends to determine the source strength at $\left(r_{0}, z_{0}\right)$ $=(0,2) \mathrm{mm}$. The reader is referred to Section 3.1 for the precise geometry. Because dimensions of ophthalmic applicators are significantly smaller than those of line sources and because it is difficult to construct a phantom that allows averaging, the NCS recommends to use a point dose rate for determination of the source strength $\dot{D}\left(r_{0}, z_{0}\right)$. 


\section{Source non-uniformity}

\section{Overview of literature}

The source non-uniformity for eye applicators, as introduced in the ICRU report, is given by Equation 3-1. The report recommends that the non-uniformity should be determined at a specified depth over a specified area of the source. For planar sources the concept of field center can be used [33]. Although the principle can also be used for measurements with a larger detector such as a diode, this concept is especially suitable for scanned radiochromic films. The ICRU proposes to determine the non-uniformity at $z=1 \mathrm{~mm}$ depth, using $80 \%$ of $R_{50}$. The calculation of the source non-uniformity is performed as follows:

- The maximum absorbed dose rate $\dot{D}_{\max }$ is determined.

- The pixels with $50 \%$ of $\dot{D}_{\max }$ are determined (for films, absorbed dose values are used, instead of dose rate values).

- The coordinate of the field center $\left(x_{c}, y_{c}\right)$ is determined through:

$x_{c}=\left(\frac{1}{n}\right) \sum_{i=1}^{n} \frac{x 50_{i}^{-}+x 50_{i}^{+}}{2}, y_{c}=\left(\frac{1}{m}\right) \sum_{j=1}^{m} \frac{y 50_{j}^{-}+y 50_{j}^{+}}{2}$

In which $n$ is the number of rows and $m$ is the number of columns with a dose rate larger than or equal to $50 \%$ of $\dot{D}_{\text {max }}$. The sign indicates the position with respect to the source center.

- The average radius $R_{50}$ can be calculated through:

$$
R_{50}=\left(\frac{1}{q}\right) \sum_{k=1}^{q} \sqrt{\left(x_{c}-x 50_{k}\right)^{2}+\left(y_{c}-y 50_{k}\right)^{2}}
$$

in which $q$ is the total number of $50 \%$ pixels.

- Finally, analogous to the concept for line sources, Equation 3-1 is applied, in which $\dot{D}_{\text {min }}$, $\dot{D}_{\max }$ and $\dot{D}_{\text {avg }}$ are taken from the pixels with a distance smaller than $0.8 R_{50}$ to $\left(x_{c}, y_{c}\right)$. The ICRU recommends a maximum source non-uniformity $U_{I C R U}$ of $20 \%$.

For concave sources the concept of non-uniformity can be applied in different ways:

1) in a concave surface parallel to the source surface,

2) in a plane perpendicular to the source axis.

Although uniformity measurements should preferably be performed parallel to the source surface, film measurements are only possible in a plane. In the literature, film measurements on concave ophthalmic sources are described [34] and also in this report results of such film measurements will be presented. However, these measurements combine effects of nonuniformity and the non-constant distance between the concave source surface and the film. Measurements with a small detector parallel to the source surface are also presented in this report.

\section{NCS definition}

The concept of non-uniformity proposed by the ICRU has some disadvantages.

1) The non-uniformity does not give the maximum possible deviation from the source strength, but only the difference between minimum and maximum.

2) The normalization is performed based on an average of the absorbed dose within the area of interest and can therefore not be related directly to the source strength that is defined in a point on the axis. 
For these reasons, the NCS proposes to use a concept in which the non-uniformity gives a percentage value of the maximum deviation from the source strength. This source strength is defined on the source axis as described earlier. The following equation is applied for the source non-uniformity (flatness):

$U_{F}=\max \left(\left|\dot{D}_{\min }-\dot{D}_{r=0}\right|,\left|\dot{D}_{\max }-\dot{D}_{r=0}\right|\right) / \dot{D}_{r=0} \times 100 \%$

$\dot{D}_{r=0}$ is the (relative) absorbed dose rate as determined on the z-axis $(r=0)$ and may be equal to the source strength $\dot{D}\left(r_{0}, z_{0}\right)$. The other variables are equal to those introduced by the ICRU. Again, the area within $0.8 \mathrm{R}_{50}$ is used for evaluation of source non-uniformity. A direct relation with source strength is obtained when non-uniformity is determined at the reference distance of $2 \mathrm{~mm}$ from the surface. Detector positioning at $2 \mathrm{~mm}$ is often accompanied by additional uncertainties in the measurement setup. From a clinical point of view, the measurement distance should preferably be less than $2 \mathrm{~mm}$, because problems caused by hot spots probably will occur close to the source surface. Because of these reasons, the NCS recommends performing uniformity measurements at a distance as close to the surface of the source as possible. The relatively small effect of distance on the magnitude of the non-uniformity is accounted for in the tolerances that will be given in Chapter 6 for the non-uniformity. The non-uniformity is defined parallel to the source surface.

It is possible to generate radial plots of the absorbed dose in which the flatness and symmetry of the activity distribution can be evaluated. Figure 3-3 shows an example in which the radial dose distribution of a film measurement on a planar source is given (in practice absorbed dose is measured with film and not the dose rate). In this figure every pixel is displayed as a dot. Because the number of pixels is proportional to the surface, it increases with increasing radius.

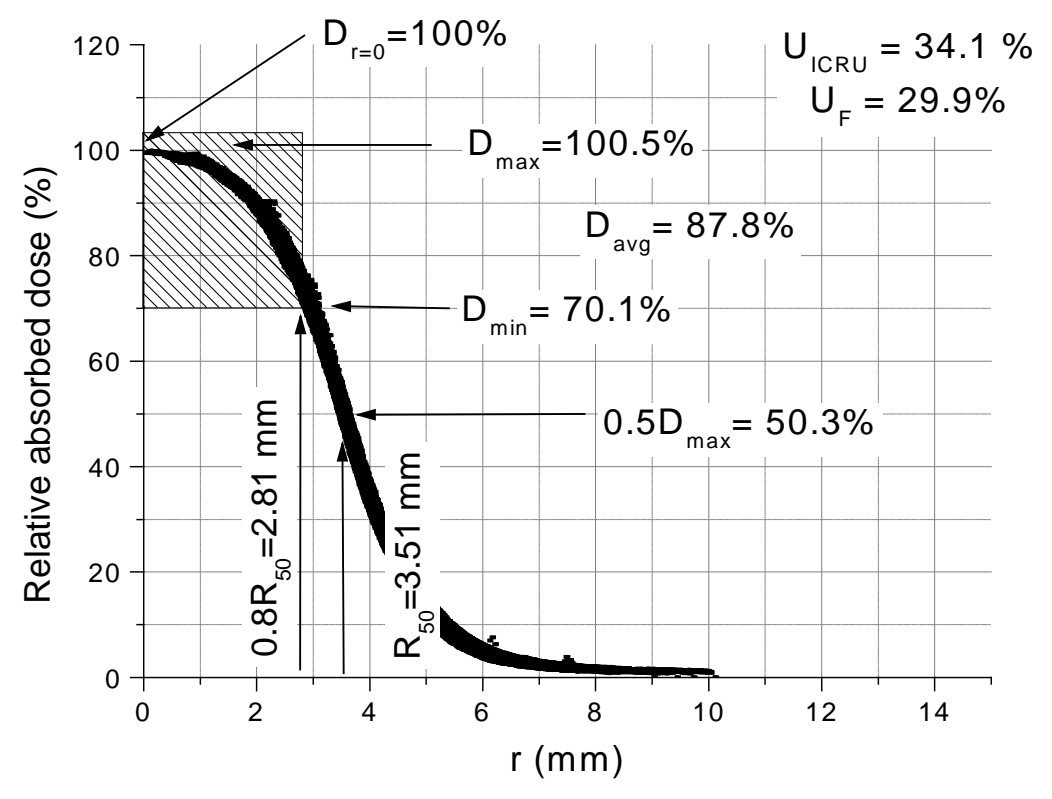

Figure 3-3 Radial dose distribution at the surface of a SIA.20 planar source. The shaded area of the figure contains the data points used for the non-uniformity calculation. 
The results of the experiments described in Chapter 5 show that many of the clinical sources do not satisfy the ICRU criterion of a maximum non-uniformity of $20 \%$. 4 out of 5 planar strontium sources and 5 out of 10 concave ruthenium sources display a non-uniformity that is too high. It is therefore justified to state that the ICRU criterion is not in tune with clinical practice. Demands on uniformity should be in line with clinical practice, especially when treatment results are considered to be acceptable. It may even be questioned whether the use of sources with a flat activity distribution is desirable. For concave sources, used for the irradiation of eye melanoma, it may be beneficial to have a somewhat higher dose rate close to the source edge in order to achieve better depth dose characteristics. Based on these considerations, it was decided to use a wider margin for the non-uniformity and to introduce an additional parameter for evaluating sources with respect to the asymmetry of the dose distribution.

\section{Source asymmetry - a new NCS concept}

To quantify the asymmetry of the dose distribution of ophthalmic sources, the parameter for source asymmetry $U_{A S}$ is introduced:

$$
U_{A S}=\max \left(\left\{\dot{D}_{\max }(r)-\dot{D}_{\min }(r)\right\} / \dot{D}_{\text {avg }}(r)\right) \times 100 \%
$$

In this expression the variation of the dose rate is calculated over a circle with a radius $r$. The maximum of this variation, with $r$ ranging from zero to $0.8 R_{50}$, determines the value of the asymmetry $U_{A S}$. The asymmetry is defined parallel to the source surface. For concave sources, a film measurement in a plane may be used as an approximation of $U_{A S}$.

\subsection{Beta detectors}

\subsubsection{Introduction}

In this section, detectors for absorbed dose measurements on beta sources are described. In general the measuring techniques can be divided into three categories:

- Ionization chambers: extrapolation chamber, well-type ionization chamber, plane-parallel ionization chamber

- Radiochemical detectors: radiochromic film, polymer gels

- Solid state detectors: thermoluminescent dosemeter (TLD), plastic scintillator, diamond, alanine, diode detector

\subsubsection{Well-type ionization chamber}

The well-type or re-entrant ionization chamber is an ionization chamber with a well-shape. In radiotherapy it is the most frequently used method for calibrating line sources or seeds [3537]. The detection volume surrounds the source, resulting in a high detection efficiency compared with other ionization chambers. Well-type ionization chambers can be either open (vented) or pressurized. Pressurized chambers are filled with for example argon to increase the chamber sensitivity to photons. In contrast to pressurized chambers a temperature and pressure correction needs to be applied to open chambers. Well-type ionization chamber measurements do not provide spatial information. Usually, measurements are performed at the sweet spot of the chamber, where the response is at a maximum. The length of the "sweet spot" is one of the important characteristics of a well-type ionization chamber. The measurements are relatively simple and reliable (expanded standard uncertainty in the activity of $5 \%(2 \sigma)[36])$. The well-type ionization chamber was originally designed for measuring source activity. A conversion factor can be used to translate the activity to an absorbed dose rate in water. The dose rate at the reference point is calculated from the 
ionizations collected in the detection volume around the entire line source, under the assumption of a perfectly uniform activity distribution throughout the source.

The insert that is used to fix the source at the measurement position is part of the calibration and- for beta sources - determines to a large extent the signal strength and whether mainly beta radiation or bremsstrahlung is detected within the ionization volume. Different detector inserts have been compared by Standard Imaging (Middleton, WI, USA) and the University of Wisconsin [38]. It was concluded that the use of a $2 \mathrm{~mm}$ acrylic insert was the optimum between signal strength and reproducibility. This insert blocks clinically irrelevant low-energy betas and shows high reproducibility during the measurements.

\subsubsection{Plane-parallel ionization chamber}

The use of parallel plate ionization chambers has been described for measurements on planar strontium sources [34,39,40]. Because of the large electrode size (at least $5 \mathrm{~mm}$ ), these chambers are not suitable for characterization of the activity distribution of a source. In most reported studies the chamber is used as a relative instrument for depth dose measurements.

\subsubsection{Radiochromic film}

Radiochromic film has turned into the most widely used method for verification of the uniformity of beta sources. GafChromic ${ }^{T M}$ (ISP Technologies Inc., Wayne, NJ, USA) is available in single and double layer versions. A thin layer of almost colorless emulsion is coated on a Polyethylene TerePhthalate (PTP) backing, forming a single or double sandwich of layers. The sensitive emulsion layer is between 7 and $40 \mu \mathrm{m}$ thick, depending on the type of film. The total thickness can be between 0.1-0.3 mm (Table 3-1).

Table 3-1 Overview of currently available types of GafChromic film: MD55-2, HD810, HS, XR-R and $X R-T$.

\begin{tabular}{|l|l|l|l|l|l|}
\hline & MD55-2 & HD810 & HS & XR-R & XR-T \\
\hline Dose range (Gy) & $3-100$ & $50-2500$ & $0.5-40$ & $0.2-30$ & $0.2-15$ \\
\hline Film thickness $(\mu \mathrm{m})$ & 278 & 107 & 230 & 210 & 220 \\
\hline $\begin{array}{l}\text { Thickness of } \\
\text { sensitive layer }(\mu \mathrm{m})\end{array}$ & $2 \times 15$ & 7 & 40 & 15 & 18 \\
\hline Specific features & $\begin{array}{l}\text { 2 sensitive } \\
\text { layers }\end{array}$ & $\begin{array}{l}\text { Flexible, thin, } \\
\text { sensitive layer } \\
\text { on surface }\end{array}$ & $\begin{array}{l}\text { Flexible, } \\
\text { no adhesive } \\
\text { layer }\end{array}$ & $\begin{array}{l}\text { Reflective, } \\
\text { addition of low } \\
\text { Z material }\end{array}$ & $\begin{array}{l}\text { Transparent, } \\
\text { addition of low } \\
\text { Z material }\end{array}$ \\
\hline
\end{tabular}

The sensitive layer turns blue upon irradiation (MD55-2, HD810, HS) and does not require post-irradiation processing. The sensitivity of most films is low, so that an absorbed dose of at least tens of Grays is needed. Films can be calibrated for absolute measurements [41]. The energy dependence is low in the energy range relevant for clinical beta sources (100 $\mathrm{keV}-2 \mathrm{MeV}$, [42]). A major limitation of the radiochromic film is the variation in thickness of the sensitive layer resulting in a non-uniform response (up to 15\% for MD55-2 [43]). It is possible to compensate for these variations by using a double exposure technique [42]. For absolute measurements a delay of at least $24 \mathrm{~h}$ needs to be taken into account to minimize effects of incomplete film development. Uniformity information can be quantified by film densitometry [42]. In Table 3-2 an overview is given of frequently used densitometers. The performance of black and white densitometers can be enhanced some $50 \%$ by using a deep orange colored filter ${ }^{*}$ when scanning the film [44]. Uniformity variations of more than $\sim 10 \%$ can be detected (qualitatively) by the eye.

\footnotetext{
Such a filter is available through Nuclear Associates (Hicksville, NY, USA).
} 
In the literature radiochromic film measurements are described for both ophthalmic $[45,46]$ and intravascular $[41,47]$ sources. Film measurements can also be performed on concave applicators $[46,48]$.

Table 3-2 Frequently used densitometers (as well as the Microtek flatbed scanner used in this report)

\begin{tabular}{|c|c|c|c|c|c|c|}
\hline Company & Model & $\begin{array}{c}\text { Light source type } \\
\text { (wavelength) }\end{array}$ & Detector & $\begin{array}{c}\text { Signal } \\
\text { resolution }\end{array}$ & $\begin{array}{c}\text { Density } \\
\text { range }\end{array}$ & Used by: \\
\hline $\begin{array}{c}\text { LKB } \\
\text { Pharmacia }\end{array}$ & UltroScan XL & $\begin{array}{c}\text { He-Ner laser } \\
(633 \mathrm{~nm})\end{array}$ & & 12 bit & $0-4.0$ OD & $\begin{array}{c}\text { NIST [33,49], } \\
\text { Duggan [50], } \\
\text { Kirisits [32] }\end{array}$ \\
\hline Lumisys & $\begin{array}{c}\text { Lumiscan } 75 \\
\text { or 150 }\end{array}$ & $\begin{array}{c}\text { laser } \\
(633 \mathrm{~nm})\end{array}$ & $\begin{array}{c}\text { Linear } \\
\text { CCD }\end{array}$ & 12 bit & $0-3.5$ OD & Gluckman [51] \\
\hline $\begin{array}{c}\text { Vidar } \\
\text { Systems } \\
\text { Corporation }\end{array}$ & $\begin{array}{c}\text { VXR-12 or } \\
\text { VXR-16 }\end{array}$ & $\begin{array}{c}\text { Standard fluorescent } \\
\text { light (broadband) }\end{array}$ & $\begin{array}{c}\text { Linear } \\
\text { CCD }\end{array}$ & $\begin{array}{c}12 \text { or 16 } \\
\text { bit }\end{array}$ & $0-2.6$ OD & $\begin{array}{c}\text { Gluckman [51], } \\
\text { Piessens [41], } \\
\text { Häfeli [52], Odero } \\
{[44]}\end{array}$ \\
\hline $\begin{array}{c}\text { Nuclear } \\
\text { Associates }\end{array}$ & $\begin{array}{c}\text { Radiochromic } \\
\text { Densitometer }\end{array}$ & $\begin{array}{c}\text { Red LED } \\
\text { (10 nm bandwidth } \\
\text { centered at 671 nm) }\end{array}$ & $\begin{array}{c}\text { Single spot } \\
\text { silicon } \\
\text { photodiode }\end{array}$ & $\begin{array}{c}3 \text { digit OD } \\
\text { output }\end{array}$ & $0-4.0$ & Gluckman [51] \\
\hline Microtek & $\begin{array}{c}\text { ScanMaker } \\
9600 X L \text { flatbed }\end{array}$ & White light & $\begin{array}{c}\text { Linear } \\
\text { CCD }\end{array}$ & 12 bit & $0-3.2$ OD & (NCS report) \\
\hline
\end{tabular}

\subsubsection{Polymergel}

In a few cases BANG® polymer gels (MGS Research, Guilford, CT, USA) have been used for 3D-dosimetry of line sources [52] or ophthalmic plaques [53]. The technique is based on the radiation induced free-radical chain polymerization of acrylic monomers. The NMR relaxation of water protons in the gel is strongly affected by this polymerization. The proton relaxation rate $(\mathrm{R} 2)$ of water protons in the polymerized gel increases linearly with absorbed dose, independent of dose rate or radiation quality. After irradiation, 3D dose maps can be created based on R2 maps calculated from MRI spin-echo images [54,55]. In a single measurement the full dose map of a source can be registered. For a ruthenium plaque an irradiation time of $\sim 1 \mathrm{~h}$ is needed [53].

The technique is highly sensitive to environmental conditions such as temperature, light and oxygen concentration. The resolution of the MRI scanner is one of the bottlenecks of the measurement. The introduction of a dedicated microscopic 3D optical density scanner for this application would significantly improve resolution and reduce environmental dependence [55].

\subsubsection{Thermoluminescent dosemeter (TLD)}

Although most hospitals make use of TLDs for dosimetry, dedicated TLDs are required for measurements on beta sources $[34,40,41,56]$. The most important property of these TLDs is their small size (as small as $0.1 \mathrm{~mm}$ thickness and $1 \mathrm{~mm}$ diameter for the active volume), which makes them suitable for determining the spatial distribution of the dose around a source. Due to their small size and water-equivalence these TLDs give only very small distortions of the radiation field. The TLDs are usually embedded in a solid phantom. Readout is performed afterwards by heating the TLDs and measuring the amount of light with a photomultiplier. Calibration is carried out in a clinical $\mathrm{MeV}$ electron beam or with a calibrated beta source. TLDs can also be used for measurements on concave ophthalmic applicators $[30,57,58]$. The estimated standard uncertainty $(1 \sigma)$ is between $8-10 \%$ depending on knowledge of precise phantom dimensions and TLD characteristics [34]. 


\subsubsection{Plastic scintillator}

Plastic scintillators were first introduced for dosimetry of brachytherapy sources at the University of Essen. The detector is based on light being generated in the plastic scintillator volume. The scintillation light is detected with a photomultiplier through an optical fiber. The signal from a blind fiber can be used to correct for Cherenkov light, created within the optical fiber. Plastic scintillators are attractive because of their water equivalence and the small detection volume. The Essen plastic scintillator has a thickness of $0.4 \mathrm{~mm}$ and a diameter of $1 \mathrm{~mm}$. Measurements on ophthalmic applicators $\left({ }^{125} \mathrm{I}\right.$ and $\left.{ }^{106} \mathrm{Ru} /{ }^{106} \mathrm{Rh}\right)$ showed a contribution of Cherenkov light of no more than $2 \%$. This system has been used for measurements on both ophthalmic plaques [59] and intravascular brachytherapy sources [60-62].

The Optidos ${ }^{\mathrm{TM}}$ is a commercial version of this system developed by PTW (PTW Freiburg $\mathrm{GmbH}$, Freiburg, Germany). The plastic scintillator has a detection volume of $0.8 \mathrm{~mm}^{3}(1 \mathrm{~mm}$ thickness and $1 \mathrm{~mm}$ diameter) and an outer diameter of $5.2 \mathrm{~mm}$. The system has no blind fiber for Cherenkov compensation. Measurements need to be accompanied by check source measurements to correct for changes in the detector sensitivity. The estimated standard uncertainty $(1 \sigma)$ is $3 \%$ for relative dosimetry with the larger plastic scintillator without Cherenkov compensation and $7 \%$ for the smaller scintillator with Cherenkov compensation [34].

\subsubsection{Diamond detector}

The diamond detector is based on radiosensitive resistance of the detection material. One of the characteristics of the detector is its high sensitivity. The variation of the stopping power ratio to water in the range $0.02-5 \mathrm{MeV}$ is less than $2 \%$. Diamond detectors are produced individually and their exact dimensions may vary. The detection volume is several $\mathrm{mm}^{3}$, but the exact position of the detection volume is one of the larger contributors to the overall estimated standard uncertainty of $10 \%(1 \sigma)$ [34]. Dose rates higher than $25 \mathrm{mGy} \mathrm{s}^{-1}$ are underestimated, because of electron-hole recombination [34]. Pre-irradiation with 5-10 Gy is required to obtain a reliable signal. The detector can be used for measurements on concave ophthalmic applicators [63], despite the large outer diameter ( $7 \mathrm{~mm}$ according to PTW specifications) [64].

\subsubsection{Diode detector}

Most semiconductor detectors are based on p-type silicon, on one side doped with n-type material in order to create a $p-n$ junction or a diode. During irradiation a current is present in the depletion layer (effective thickness of about $60 \mu \mathrm{m}$ ). There is significant variation in stopping power ratio between silicon and water (up to $8 \%$ between 0.5 and $3.5 \mathrm{MeV}$ ) [65]. Pre-irradiation with about $5 \mathrm{kGy}$ by the manufacturer is needed for reliable response [66]. The standard uncertainty is estimated to be $5 \%(1 \sigma$ or $2 \sigma, \mathrm{k}$ is not quoted) [29]. With an outer diameter of 4-7 mm and a sensitive area of 1-2 $\mathrm{mm}$ (specifications Scanditronix Medical AB, Uppsala, Sweden) [29], the detector can be used for measurements on concave sources [67]. The sensitivity of the detector decreases with time, requiring regular recalibrations. Sensitivity decrease can be $\sim 5 \%$ in a period of 5 years [29], but depends on the intensity of use. The detector is only used for relative measurements within the daily practice of radiotherapy.

\subsubsection{Alanine detector}

Dosimetry with alanine is based on the relative and non-destructive measurement of stable free radicals produced by radiation $[34,68]$. These dosemeters are read out with an X-band Electron Paramagnetic Resonance (EPR) spectrometer with a scan range of 0-2 mT.

Advantages of the detector are the linear response and absence of energy dependence. The detector has a diameter of about $5 \mathrm{~mm}$ and a thickness of about $1 \mathrm{~mm}$. These dimensions also determine the sensitive volume and therefore are a disadvantage of the method. 
Another disadvantage is the low sensitivity, because the EPR signal is very weak. An estimated standard uncertainty of $5-7 \%(1 \sigma)$ can be expected in the region between $10-20$ Gy [34].

\subsubsection{Absolute calibration of beta sources: The NMi extrapolation chamber}

An extrapolation chamber is a suitable measuring device for determining the absorbed dose (rate) due to beta sources. The US standards laboratory NIST uses such a chamber as a primary standard for the calibration of sealed beta sources [69]. Recently, at the NMi a primary standard was built for the dosimetry of clinical sealed beta sources, based on the principle of the extrapolation chamber. A schematic view of this extrapolation chamber is shown in Figure 3-4.

The extrapolation chamber is a parallel plate ionization chamber with variable air volume. The ionization volume is determined by the distance between the parallel plates and by the effective area of the central electrode. The central electrode, surrounded by a guard electrode, is situated at the center of one of the parallel plates. The other parallel plate is the entrance window of the ionization chamber. The central and guard electrodes are constructed from an electrically conductive and water equivalent plastic resembling polystyrene, called D400 (Standard Imaging, Middleton, WI, USA). The ionization current collected by the central electrode is measured at different plate distances and extrapolated to zero air volume, in order to approximate the ideal Bragg-Gray conditions. The extrapolated ionization current is a measure for the absorbed dose to air, taking into account the geometrical conditions and the materials used to construct the extrapolation chamber.

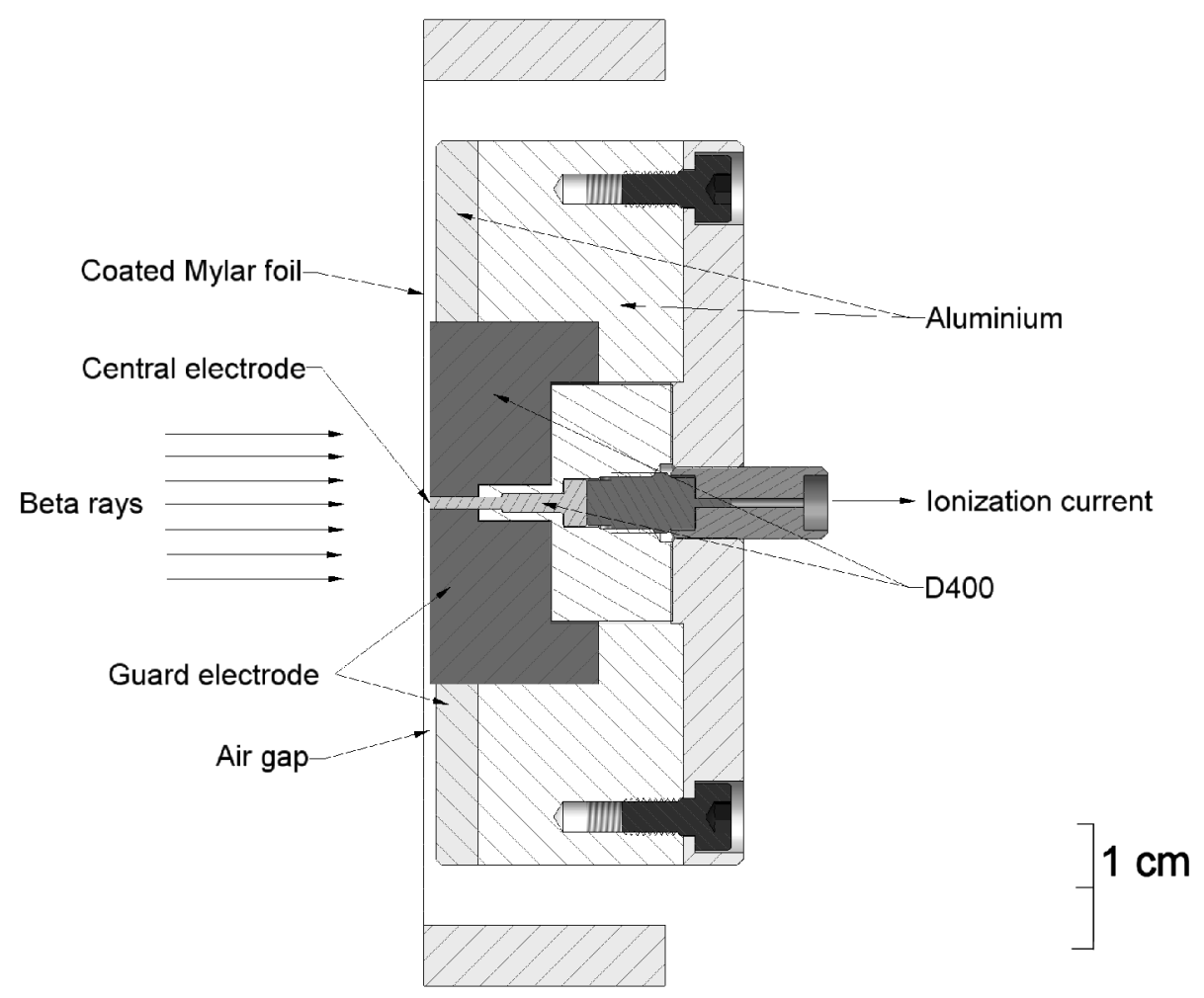

Figure 3-4 Schematic diagram of the NMi extrapolation chamber. The central and guard electrode are made of 0400 plastic (water equivalent) material.

The absorbed dose rate to water $\left(\dot{D}_{w}\right)$ can be determined from the ionization current measurements using Bragg-Gray theory and is given by Equation 3-7: 


$$
\dot{\mathrm{D}}_{\mathrm{w}}=\mathrm{s}_{\mathrm{w}, \text { air }} \frac{\overline{\mathrm{W}} \cdot \mathrm{k}_{1}}{\mathrm{e} \cdot \mathrm{A} \cdot \rho_{0}} \times\left(\frac{\mathrm{d}\left(\mathrm{k}_{2} \times \mathrm{I}_{\mathrm{av}}\right)}{\mathrm{dx}}\right)_{\mathrm{x} \rightarrow 0}
$$

where: $\mathrm{s}_{\mathrm{w}, \text { air }} \quad=$ ratio of the mean mass-collision stopping power of water to that of air

$\bar{W} \quad=$ average energy required to produce an ion pair in air $(\mathrm{J})$

e $\quad=$ electron charge $(C)$

A $\quad=$ effective electrode area $\left(\mathrm{m}^{2}\right)$

$\rho_{0} \quad=$ density of air at reference conditions of temperature and pressure $\left(\mathrm{kgm}^{-3}\right)$

$\mathrm{x} \quad=$ parallel plate separation $(\mathrm{m})$

$\mathrm{l}_{\mathrm{av}} \quad=$ ionization current, corrected for polarity effect $(\mathrm{A})$

$\mathrm{k}_{1} \quad=$ product of correction factors independent of electrode separation

$\mathrm{k}_{2} \quad=$ product of correction factors dependent of electrode separation

The correction factors and geometrical constants, grouped in the products $\mathrm{k}_{1}$ and $\mathrm{k}_{2}$, are needed to convert the current measurement into absorbed dose rate to water satisfying Bragg-Gray conditions [69]. The major correction factors are the differences in scatter and absorption of beta particles between the materials of the standard and pure water. To keep these factors as small as possible, D400 is chosen as an electrode material and the entrance window is constructed of a $12 \mu \mathrm{m}$ thick foil of single sided aluminium coated mylar.

The primary standard is intended for the calibration of both ophthalmic [70] and intravascular [47] beta sources. To be able to determine the absorbed dose and absorbed dose distributions over the clinical relevant parts of the sources, high spatial resolution is required and a central electrode with an effective diameter of $1 \mathrm{~mm}$ is used. This also fulfils the requirement that the diameter of the central electrode has to be small compared with the range of the beta particles in air. Larger electrodes are also available.

Sources can be placed in a source holder in the case of ophthalmic applicators, or in a water equivalent phantom in the case of intravascular sources. The source holder or phantom can be mounted on a motorized xyz-translation table, so that by moving the source with the table a direct scan of the absorbed-dose rate distribution can be made. To calibrate beta sources, the surface of the source or phantom is placed directly against the entrance window, opposite the center of the central electrode. Using the xyz-translation table, this is reproducible within several microns. The planar ophthalmic applicators are calibrated in terms of absorbed dose to water at the surface of the source. It is also possible to use a spacer. In the case of calibration of concave sources, the geometrical properties of the extrapolation chamber require the use of a spacer. An additional transfer by means of e.g. film then needs to be made to find the absorbed dose at the reference distance of $2 \mathrm{~mm}$. The intravascular sources are calibrated in terms of absorbed dose to water at a depth of $2 \mathrm{~mm}$, in an infinite water (equivalent) phantom. Detectors used for sources that are frequently exchanged may be calibrated by means of a clinical transfer source. If applicable, the phantom is part of the calibration chain.

\subsection{Measurement corrections}

Because none of the detectors is a real point detector, corrections need to be applied in order to know the precize position of the effective point of measurement. However, for most of the experiments, exact information on the effective point of measurement is not necessary. A reproducible configuration with phantom and detector, specifically calibrated for the source type of interest, is enough for source calibration. Only for measuring the 3D dose distribution of the source more information is required. For such measurements the offset depth of the detector, effective point of measurement, correction for detector and source geometry need to be taken into account. The IAEA report describes these corrections in more detail [3]. 
Many measurements are performed in a solid phantom instead of in water. The depth dose curve in a solid phantom is in general different from that in water and therefore a correction is required. Monte Carlo calculations, in which the phantom medium is replaced by water, can be used for such corrections. Another possibility is to correct through scaling. In the literature different reviews of scaling rules are given [2,3,65], mostly based on the work of Cross for point sources [71]. Schaart has adapted the method for line sources [72]. An overview of the information that is relevant for depth dose curves of beta sources is given in Appendix 3.

Estimates of other disturbances can often be found in the manual of the detector. Possible factors are energy dependence (stopping-power ratio in the area of interest), directional dependence and dependence on temperature and/or pressure. For ionization chambers the correction through $\frac{\mathrm{T} \times \mathrm{p}_{0}}{\mathrm{~T}_{0} \times \mathrm{p}}$ is well known in radiotherapy.

\subsection{Considerations for detector selection criteria}

The required degree of accuracy in beta dosimetry should be weighted against tolerances and uncertainties in the clinical application. While a relatively high degree of accuracy is required in teletherapy and brachytherapy with gamma sources, several reasons may justify less stringent demands on the accuracy in brachytherapy with beta sources.

1) Positioning uncertainties within the patient are often in the order of 0.1-1 mm or even more. Large uncertainties may for example exist if a non-centered catheter is used for intravascular brachytherapy. A change of $0.1 \mathrm{~mm}$ in radial source positioning causes dose differences in the order of $10 \%$.

2) Relatively little is known about the minimum absorbed dose needed to achieve local control in the three applications of beta sources: in-stent restenosis [16], pterygium [25] and eye melanoma. The clinical results, however, show relatively high local control rates. In addition, few complications related to overdose - such as restenosis or cataract - have been reported [73,74]. A surface dose of $1000 \mathrm{~Gy}$ is regarded as a limitation for the irradiation of eye melanoma [29].

3) Absolute source calibrations have always been accompanied by large uncertainties of up to $30 \%(2 \sigma)$.

In the past, large uncertainties were present in the dosimetry of beta sources. This can be improved by the introduction and standardization of more accurate measurement methods in clinical practice. The methods for source strength determination should be based on - or traceable to - well established (primary) standards for absorbed dose. The measurement uncertainties should be reduced as much as possible, provided that the required investments remain reasonable. The NCS estimates that deviations in source strength below $10 \%$ will have little clinical significance compared with other factors. Uncertainties in the dosimetry of beta sources should therefore be such that a deviation of $10 \%$ can be detected within all combined measurement uncertainties that are present.

In the choice of detectors, aspects such as detector volume, reproducibility, stability, sensitivity, dependence of dose rate as well as angle of incidence and energy play a role. Other factors such as frequency of use, costs, ease of use, and availability of detectors and phantoms should also be taken into account before a measurement system is bought for dosimetry of beta sources. For calibration of sources with a long lifetime, services offered by specialized institutes - such as the NMi - may be an efficient way of handling the responsibility of independent strength and uniformity verifications. 


\subsection{Selection of detectors}

Based on the criteria mentioned in the previous paragraph, a first selection was made of detectors to be tested during a dosimetry intercomparison study as described in Chapter 5 . The considerations for this selection are given below. The availability of detectors at local departments was investigated separately and is provided in Section 4.3.

\subsubsection{Detectors that were not selected for the intercomparison}

\section{Extrapolation chamber}

The extrapolation chamber is the primary standard for beta dosimetry. It is not commercially available with electrodes smaller than $1 \mathrm{~cm}$.

\section{Polymergel}

The use of polymer gel is still very uncommon within the daily practice of radiotherapy.

Disadvantages are the strong environmental dependence and the offline measurements. The availability of read-out devices may be a bottleneck for application of polymer gels, although desktop optical readout devices are under development.

\section{$\underline{\text { TLD }}$}

Although sensitive and reliable measurements can be made with TLDs, the processing time is an important disadvantage of this method. Furthermore, TLDs that have been used for dosimetry of beta sources are different in size from the regular TLDs in radiotherapy and are not readily available. The TLDs only give information for one point; for measuring the spatial distribution around the source more TLDs are required.

\section{Diamond detector}

The required pre-irradiation and possible underestimation of the dose rate are disadvantages of this detector. The detector is not part of the standard equipment, although it is (limited) commercially available. The diameter of the active volume of $2-4 \mathrm{~mm}$ is another limitation for these measurements.

\section{Alanine}

Few institutes have experience with this detector. The low sensitivity, offline measurements and limited availability are disadvantages.

\subsubsection{Detectors selected for the intercomparison}

\section{Well-type ionization chamber}

The well-type ionization chamber is widely used for the calibration of line sources and is known for its high reproducibility. All Guidant users are equipped with a well-type ionization chamber. A disadvantage of the detector is that an additional measurement is required for determination of the source non-uniformity.

\section{Plastic scintillator}

This versatile detector is suitable for measurements on high and low dose rate sources and for strength, depth dose and uniformity measurements. It also has a high degree of water equivalence and a relatively small detector volume. It is generally available and routinely used by Novoste for source strength and uniformity verifications on their line sources.

\section{Plane-parallel ionization chamber}

The use of this detector for strength and depth dose measurements has been described as mentioned above. This detector is available in most radiotherapy departments. Size and sensitivity of the detector are factors that limit its use to high dose rates and relatively large sources such as the planar strontium applicators. 


\section{Diode}

This detector is used in many institutes for relative measurements of the dose distribution of accelerator beams. Its use is also mentioned in the literature and the detector has a relatively high spatial resolution. In practice, the detector is rarely used for absolute measurements.

Radiochromic film

Radiochromic film is widely used for uniformity measurements on beta sources. Guidant users are provided with film by the manufacturer. The film is easy to use, but quantitative evaluation is more elaborate, as densitometry has to be applied. 


\section{Current status of dosimetry of beta sources}

This chapter gives an overview of beta sources and related dosimetry in the Netherlands and Belgium. A questionnaire was sent to all radiotherapy departments to collect data on sources used and dosimetry procedures performed.

\subsection{Questionnaire}

A questionnaire was composed and sent to all known 47 radiotherapy departments in the Netherlands and Belgium (all 21 departments in the Netherlands, 26 in Belgium). The questionnaires were filled in between October 2001 and February 2002. The response was $100 \%$.

The questionnaire consisted of 3 parts:

- A list of sources (available $y / n$ )

- A list of detection methods (available $y / n$ )

- Type and frequency of current dosimetric checks

\subsection{Use of beta sources in the Netherlands and Belgium}

About half of the radiotherapy departments in the Netherlands and Belgium make use of sealed beta sources: 10/21 in the Netherlands and 13/26 in Belgium. Only sources that are commercially available and that are used clinically were included in the questionnaire.

The first part of the questionnaire concerned the source types. A general overview of all beta sources in the Netherlands and Belgium is given in Figure 4-1. Sources used for intravascular brachytherapy are the Guidant $20 \mathrm{~mm}$ and Novoste 30,40 and $60 \mathrm{~mm}$ sources. The ophthalmic sources are mostly planar and concave ${ }^{90} \mathrm{Sr} /{ }^{90} \mathrm{Y}$ applicators manufactured by Amersham. Only 3 institutes make use of concave ${ }^{106} \mathrm{Ru} /{ }^{106} \mathrm{Rh} B E B I G$ sources for the treatment of malignant eye diseases.

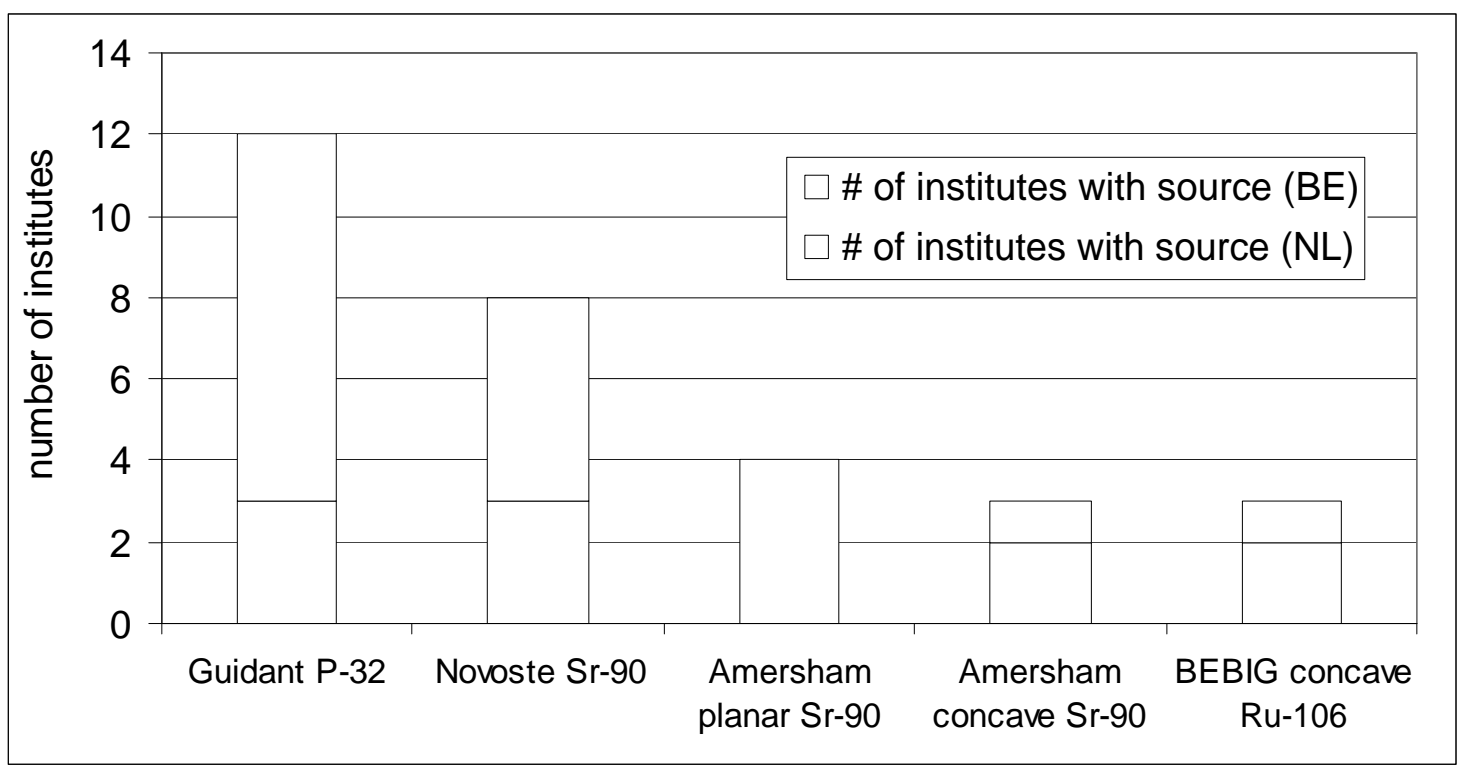

Figure 4-1 Number of institutes with one or more Guidant, Novoste, Amersham or BEBIG sources in the Netherlands and Belgium. 


\subsection{Available detectors}

The second part of the questionnaire concerned the available (in-house) detectors. Figure 4-2 shows the results for all users. The availability of detectors is given for each user group. All Guidant users are supplied with a well-type ionization chamber and radiochromic film (MD55-2 + phantom) by the manufacturer. The Dutch Novoste systems are calibrated with the Optidos plastic scintillator system (PTW) that Novoste provides with each source exchange [62]. This is not included in the graphs, because this plastic scintillator is not an inhouse detector. Most institutes have Vidar or other types of scanners that can be used for radiochromic film densitometry. At the time of the questionnaire only one institute in both countries had a plastic scintillator.

Some users of ophthalmic applicators have experience with radiochromic film (MD55-2) and have facilities for film densitometry (Lumisys, Vidar, flatbed). None of them has an inhouse plastic scintillator system. Whether or not a diode is available was not part of the questionnaire, but it is assumed that most radiotherapy institutes have a diode as part of the standard dosimetry equipment.

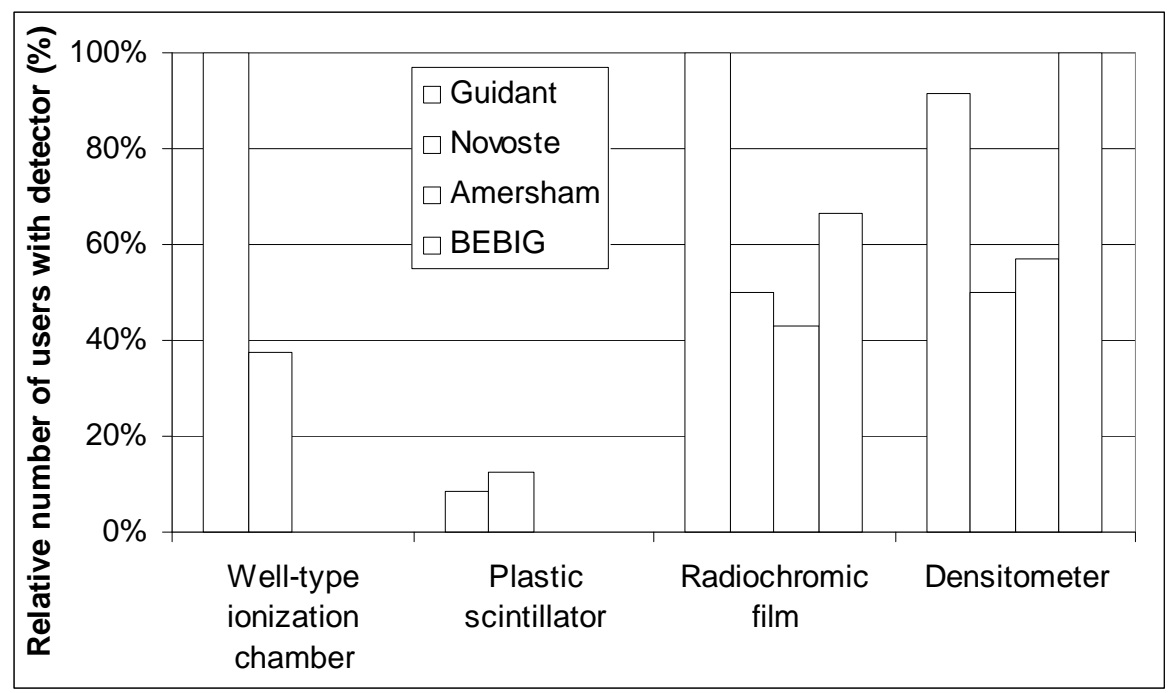

Figure 4-2 Available detectors for the different user groups of beta sources.

\subsection{Frequency of dosimetric checks}

Not all users of beta sources perform dosimetric checks on the sources. The dosimetry that is performed during source exchange is displayed in Figure 4-3. All Guidant users perform well-type ionization chamber measurements in order to verify source strength. Although all have radiochromic film available for uniformity checks, this verification in not performed by all.

Most of the Dutch Novoste users rely on the checks with the Optidos plastic scintillator system carried out by Novoste (included in figure). Most Belgian users perform their own calibrations (mostly well-type ionization chamber and film measurements), although some rely on the dose rate specified by the manufacturer. Radiochromic film measurements are used not only for determination of source uniformity, but also for absolute measurements. As far as known all institutes use the dose rates as specified on the certificate for treatment planning. 


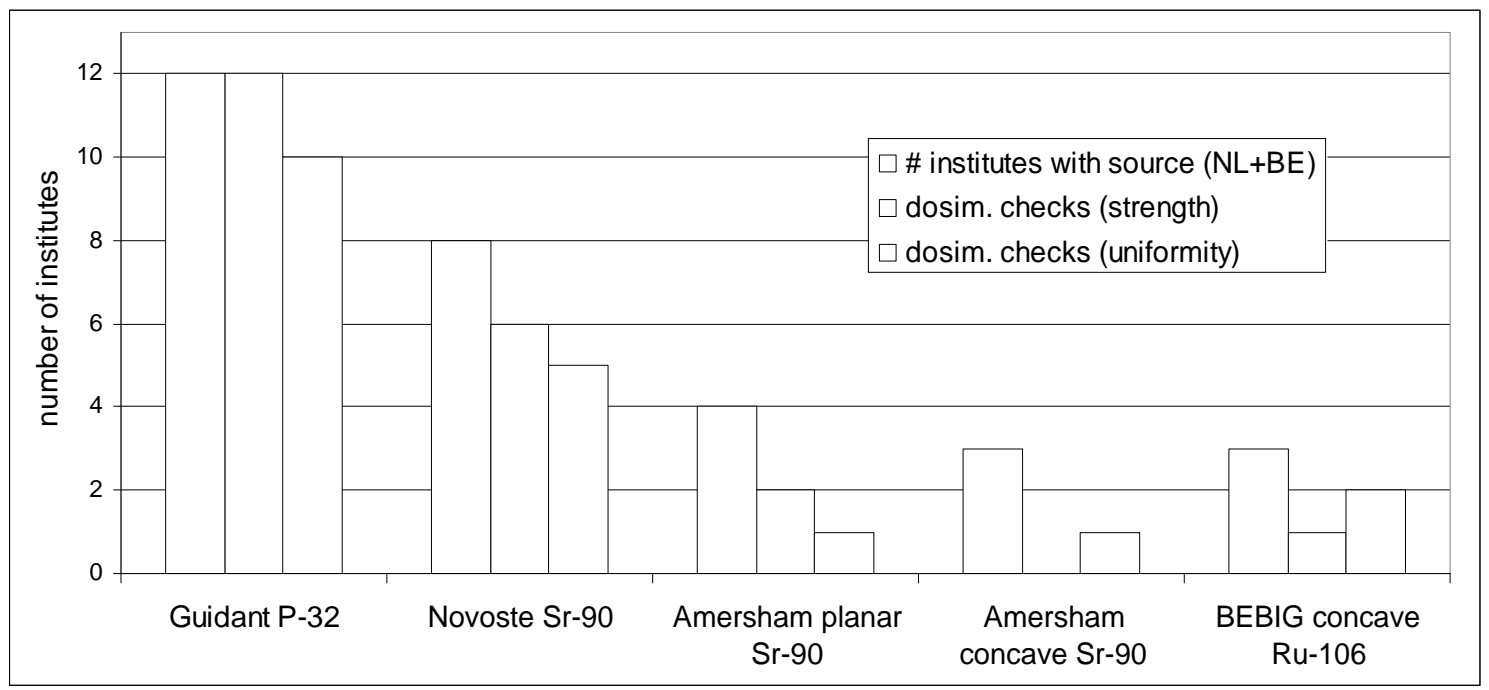

Figure 4-3 Number of institutes with a beta source and the type of dosimetry checks (strength, uniformity) being performed.

The equipment used for dosimetry checks on ophthalmic strontium applicators does not show much consistency. Some users of strontium applicators carry out film measurements, whereas others perform source strength measurements with a plane-parallel ionization chamber as part of the acceptance testing of new sources. Often a comparison is made with the previous source. Others do not perform any dosimetry check at all. Some users of ruthenium applicators perform measurements with diode or film, but also in this group not all users carry out dosimetry checks. 


\section{Results of the dosimetry intercomparison}

A number of beta detectors were tested during the summer of 2002. The selection of these detectors is given in Section 3.6. Selection of areas of application (strength, uniformity), described in the present chapter, is also based on the considerations in Section 3.6. The materials and methods for the test measurements are described extensively in Sections 5.1 and 5.2, respectively. Testing of these detectors was separated in two steps. First, introductory measurements were performed to estimate measurement uncertainties (Section 5.3). Next, on-site measurements were performed on all clinical beta sources in the Netherlands and Belgium (Section 5.4). The usefulness of the detectors for routine application in clinical practice is discussed in the last part of this chapter, Section 5.5.

\subsection{Materials}

\subsubsection{Beta sources}

The beta sources that were investigated during on-site measurements throughout the Netherlands and Belgium are given in Table 5-1. More details of the different subtypes of the BEBIG sources are given in Appendix 2.2.

Table 5-1 Characteristics and number of clinical beta sources investigated

\begin{tabular}{|l|l|l|l|l|l|l|}
\hline & Guidant & Novoste & Novoste & Amersham & Amersham & BEBIG \\
\hline Shape & $\begin{array}{l}\text { line } \\
\text { source }\end{array}$ & $\begin{array}{l}3.5 \mathrm{~F} \text { line } \\
\text { source }\end{array}$ & $\begin{array}{l}5 \mathrm{~F} \text { line } \\
\text { source }\end{array}$ & $\begin{array}{l}\text { llanar } \\
\text { source }\end{array}$ & $\begin{array}{l}\text { concave } \\
\text { source }\end{array}$ & concave source \\
\hline Subtype & $20 \mathrm{~mm}$ & $\begin{array}{l}40+60 \\
\mathrm{~mm}\end{array}$ & $\begin{array}{l}40+60 \\
\mathrm{~mm}\end{array}$ & SIA.20 & $\begin{array}{l}\text { SIA.6 } \\
\text { or custom } \\
\text { made }(2 \mathrm{x})\end{array}$ & $\begin{array}{l}\text { 3x CCB, 2x CCA, } \\
\text { CCD, CCX, CIA, } \\
\text { COB2, CIB }\end{array}$ \\
\hline Nuclide & ${ }^{32} \mathrm{P}$ & ${ }^{90} \mathrm{Sr} /{ }^{90} \mathrm{Y}$ & ${ }^{90} \mathrm{Sr} /{ }^{90} \mathrm{Y}$ & ${ }^{90} \mathrm{Sr} /{ }^{90} \mathrm{Y}$ & ${ }^{90} \mathrm{Sr} /{ }^{90} \mathrm{Y}$ & ${ }^{106} \mathrm{Ru} /{ }^{106} \mathrm{Rh}$ \\
\hline Total number & $12 \mathrm{x}$ & $\begin{array}{l}4 \times 40 \mathrm{~mm} \\
\text { and } \\
2 \times 60 \mathrm{~mm}\end{array}$ & $\begin{array}{l}3 \times 40 \mathrm{~mm} \\
\text { and } \\
3 \times 60 \mathrm{~mm}\end{array}$ & $\begin{array}{l}2 \times 11 \mathrm{~mm} \\
\text { and } \\
3 \times \varnothing 7 \mathrm{~mm}\end{array}$ & $4 \mathrm{x}$ & $10 \mathrm{x}$ \\
\hline
\end{tabular}

\subsubsection{Detectors}

The different detectors are given in Table 5-2. In addition, the bias voltage used for the ionization chambers is given. Only one of each type of detectors was used during the measurements (except for the films). More detailed information, such as size of active volume and detector serial numbers, is available in Appendix 4.

Table 5-2 List of detectors tested and, if applicable, bias voltage.

\begin{tabular}{|l|l|c|}
\hline Detector type & Manufacturer & Bias $^{*}$ (V) \\
\hline $\begin{array}{l}\text { HDR1000 Plus well-type ionization } \\
\text { chamber }\end{array}$ & $\begin{array}{l}\text { Standard } \\
\text { Imaging }\end{array}$ & -300 \\
\hline IVB1000 well-type ionization chamber & $\begin{array}{l}\text { Standard } \\
\text { Imaging }\end{array}$ & -300 \\
\hline Optidos plastic scintillator & PTW & n/a \\
\hline GAFChromic HD810 & ISP & n/a \\
\hline GAFChromic HS & ISP & n/a \\
\hline GafChromic MD55-2 & ISP & n/a \\
\hline Markus 23343 ionization chamber & PTW & -300 \\
\hline EFD electron semiconductor detector & Scanditronix & 0 \\
\hline
\end{tabular}

*A Standard Imaging CDX-2000A electrometer was used for the ionization chambers and the diode. 
All films were scanned with a white light flatbed scanner. The areas of application (strength, uniformity or depth dose) are given in Table 5-3 for the various detectors.

Table 5-3 Overview of application of detection methods.

\begin{tabular}{|l|l|l|l|}
\hline & $\begin{array}{l}\text { Intravascular } \\
\text { brachytherapy }\end{array}$ & $\begin{array}{l}\text { Ophthalmic strontium } \\
\text { sources }\end{array}$ & $\begin{array}{l}\text { Ophthalmic ruthenium } \\
\text { sources }\end{array}$ \\
\hline Plastic scintillator & $\begin{array}{l}\text { SS, U, PDD } \\
\text { (in PMMA) }\end{array}$ & $\begin{array}{l}\text { SS, PDD } \\
\text { (in solid water, RMI-457) }\end{array}$ & $\begin{array}{l}\text { SS, PDD } \\
\text { (in solid water, RMI-457) }\end{array}$ \\
\hline Radiochromic film & $\begin{array}{l}\text { U } \\
\text { (in solid water, RMI-457) }\end{array}$ & $\begin{array}{l}\text { U, PDD } \\
\text { (in solid water, RMI-457) }\end{array}$ & $\begin{array}{l}\text { U, PDD } \\
\text { (in solid water, RMI-457) }\end{array}$ \\
\hline $\begin{array}{l}\text { Well-type ionization } \\
\text { chamber }\end{array}$ & SS & & \\
\hline Diode detector & & & U, PDD (in water) \\
\hline $\begin{array}{l}\text { Plane-parallel } \\
\text { chamber }\end{array}$ & & SS (planar sources) & \\
\hline
\end{tabular}

$S S=$ Source strength measurements; $U=$ uniformity measurements; $P D D=$ Percentage depth dose measurements.

\subsubsection{Measurement setup}

Phantoms were required for almost all measurements, except those with the well-type ionization chamber. The well-type ionization chambers had an insert with a thickness of 2.1 $\mathrm{mm}$ acrylic for the IVB1000 and $0.08 \mathrm{~mm}$ stainless steel for the HDR1000+. Catheters were used for measurements on the intravascular sources. Both catheters and phantoms are described in this section. All custom-made phantoms were made of Solid Water ${ }^{\mathrm{TM}}$ (RMI-457, Gammex-RMI, Middleton, WI, USA).

\section{Catheters for intravascular sources}

The physics coupler ${ }^{\dagger}$ that is delivered by Guidant for QA (quality assurance) measurements was used for all measurements (with film, plastic scintillator and well-type ionization chamber). Through this physics coupler the source wire can be sent from the afterloader to the phantom or the well-type ionization chamber. The coupler has an open end and a screw through which it can be connected.

A clinical catheter was used for measurements with the IVB1000 well-type ionization chamber on the Novoste sources. It had an outer diameter of 3.5F or 5F, depending on the system. The clinical $3.5 \mathrm{~F}$ catheters were used only once because of their vulnerability, the $5 \mathrm{~F}$ catheter was used more then once. They could be positioned in the insert of the IVB1000 and tightened with a screw. This chamber insert has fixed positions for 30,40 and $60 \mathrm{~mm}$ long sources, so that the source was positioned in the middle of the sweet spot of the chamber.

A dedicated physics catheter ${ }^{\ddagger}$ was used for plastic scintillator and radiochromic film measurements on the Novoste sources. This catheter had a single lumen with an inner diameter of $0.56 \mathrm{~mm}$ ( $3.5 \mathrm{~F}$ source) or $0.74 \mathrm{~mm}$ ( $5 \mathrm{~F}$ source) and an outer diameter of 1.19 $\mathrm{mm}$.

\section{Intravascular source phantoms}

PMMA phantoms were available for plastic scintillator measurements on Novoste and Guidant sources (made by PTW, see Appendix 5). These allowed measurements on opposing sides of the sources at the reference point at $z_{0}=0 \mathrm{~mm}$ and $r_{0}=2 \mathrm{~mm}$. In the

\footnotetext{
ScanMaker 9600XL, Microtek Europe (Rotterdam, the Netherlands), see also Section 3.3.4.

${ }^{\dagger}$ Guidant Galileo Physics Coupler, REF 10008296.

${ }^{\ddagger}$ Novoste Beta-Cath ${ }^{\text {TM }}$ 3.5F System Guide Tube Kit, REF GTA-0035

or Novoste Beta-Cath ${ }^{\text {TM }}$ System Guide Tube Kit, REF GTA-0050.
} 
phantom, depth dose measurements could be performed at $z=0$ using spacers. The source profile of the Guidant sources was obtained by stepping the source through the Optidos phantom. This phantom had a circular bore, in which the source runs, with a diameter of $0.60 \pm 0.02 \mathrm{~mm}$. The source profile of the Novoste sources was obtained at positions with $z=$ $0, \pm 10$ and $\pm 20 \mathrm{~mm}$. This phantom had a circular bore in which both physics catheters fitted. A film phantom was designed in which the uniformity of intravascular sources could be determined at the reference distance $r_{0}=2 \mathrm{~mm}$. The phantom was made in such a way that it could be used for measurements on both the ${ }^{90} \mathrm{Sr} /{ }^{90} \mathrm{Y}$ Novoste and ${ }^{32} \mathrm{P}$ Guidant sources. Details of the design are given in Appendix 6 . The phantom had a different insert for these two types of sources. The phantom for the Guidant sources had a square channel with a side of $0.5 \mathrm{~mm}$ (source diameter is $0.46 \mathrm{~mm}$ ), to which the physics coupler can be connected. The one for the Novoste sources had a square channel with a side of $1.2 \mathrm{~mm}$ in which the physics catheter could be inserted.

\section{Planar ophthalmic source phantoms}

Phantoms were designed for measurements on the planar ophthalmic sources using a plastic scintillator, plane-parallel ionization chamber and radiochromic film. All phantoms had the same principle for insertion of the source. A cross-section of the phantom for plastic scintillator measurements is given in Figure A11, Appendix 7. The source could be centered with respect to the detector. Spacers of different thickness could be inserted for depth dose measurements. A sketch of the setup used for measurements with the plastic scintillator and plane-parallel ionization chamber is given in Appendix 8. Similar setups were used for measurements on all ophthalmic sources (both planar and concave), except for those on ruthenium in water. Measurements with the plane-parallel ionization chamber were performed with the protective cover screwed onto the chamber. This cap has a thickness of $0.87 \mathrm{~mm}$ acrylic (1 $\mathrm{mm}$ water equivalent).

\section{Concave ophthalmic source phantoms}

Spherical phantoms were designed for measurements on the concave ophthalmic sources. They had a radius of curvature of $12 \mathrm{~mm}$ (ruthenium sources) and $10 \mathrm{~mm}$ (strontium sources). The phantoms for depth dose measurements using either radiochromic film or a plastic scintillator are given in Figure A12 and A13 of Appendix 7. In the design of the phantom for the film depth dose measurements a space of $0.2 \mathrm{~mm}$ was left open to compensate for film size. Spherical phantoms for uniformity measurements were designed with a thickness of $3.4 \mathrm{~mm}$ (strontium sources) and $4.5 \mathrm{~mm}$ (ruthenium sources) under which a film could be positioned. These distances are given by the nearest possible planes perpendicular to the source axis, outside the source geometry.

Setup diode measurements in water

Diode measurements on the ruthenium sources were performed in water. A sketch of the setup is given in Appendix 9. The source-detector distance was determined with a depth dial indicator.

\subsubsection{Check source measurements}

Check source measurements were performed to verify the stability of the plastic scintillator, plane-parallel ionization chamber and diode detector. A $33.3 \mathrm{MBq}^{90} \mathrm{Sr} /{ }^{90} \mathrm{Y}$ check source was available for this purpose (see Appendix 4 for more detail).

For check source measurements on the plastic scintillator a dedicated phantom was available from PTW. Check source measurements were performed every 40 minutes as advised by the manufacturer. These measurements were used to correct for changes in detector sensitivity.

Check source measurements on the Markus chamber were only used as a verification of detector stability. A special ring was available for placement of the check source. 
Diode measurements were performed (without a phantom) in air at the point with the maximum response in contact with the source. Due to the manual outlining of the diode above the source, the positioning accuracy was not as high as for the other check source measurements. No phantom was available at the time of the measurements.

\subsection{Measurement methods}

The measurement procedures were established before the on-site measurements for each source type. The procedures are described in detail in Appendix 10. In this section the most relevant information is given per source type. In addition, calibration, densitometry and data processing of radiochromic film measurements are provided.

\subsubsection{Measurement methods for intravascular sources}

All well-type ionization chamber measurements on the Guidant sources were performed at the sweet spot, which was determined by stepping the source through the chamber and measuring the dose rate. Three source positions for the Novoste source could be selected in the IVB1000 chamber by adjusting the insert height in the chamber: 30,40 and $60 \mathrm{~mm}$. The $40 \mathrm{~mm}$ position was used for measurements on the $40 \mathrm{~mm}$ sources and the $60 \mathrm{~mm}$ position for those on the $60 \mathrm{~mm}$ sources. For the Novoste sources, measurements in the four compass directions of the chamber were averaged, turning both insert and catheter. For the Guidant sources no rotation was possible, but the insert thickness was much thinner $(0.08$ $\mathrm{mm}$ stainless steel) and no catheter was involved. Therefore the rotational dependence of these measurements was expected to be very low. Averaging was performed over repeated source insertions for both well-type ionization chamber and plastic scintillator measurements. Plastic scintillator measurements were performed at different depths and along the source axis. For measurements on the Guidant sources $2 \mathrm{~mm}$ steps were used, for the Novoste sources the positions were dictated by the phantom (10 $\mathrm{mm}$ intervals). The possible measurement distances from the source axis are given in Appendix 5 for both source types. HD810 and HS radiochromic film were used for uniformity measurements. The effect of stepping of the ${ }^{32} \mathrm{P}$ source was investigated with $\mathrm{HS}$ in a separate measurement with two 20 $\mathrm{mm}$ steps (3 source positions). The absorbed doses that were used to irradiate the films are given in Table 5-4. Although longer irradiation times were possible with the Guidant afterloader, the HD810 film was irradiated up to 100 Gy only, because the radiation time had to be entered manually (in steps of $1 \mathrm{~s}$ ) at the console of the afterloader. No attention was paid to the orientation of the sensitive side of the HD810 film because only relative measurements were performed.

\subsubsection{Measurement methods for ophthalmic strontium sources}

During plastic scintillator and plane-parallel ionization chamber measurements repeated source insertions were carried out. During all measurements on the ophthalmic sources the active side of the source was facing downwards because of safety reasons. The only exception was the diode measurement on ruthenium sources in water.

Plane-parallel ionization chamber measurements were performed with the protective cover screwed onto the chamber to prevent damage to the electrode. The cap was regarded as part of the total setup.

Film measurements were performed on ophthalmic sources to determine the depth dose and source uniformity. The uniformity measurements on the planar sources were performed at the surface of the applicator, those on the concave sources were performed at $z=3.4 \mathrm{~mm}$. Film measurements on the planar sources were performed with HD810 and MD55-2. Those on the concave sources were performed with HS and MD55-2, because of the lower dose rate of these sources. The absorbed doses that were used to irradiate the films are given in Table 5-4. 
The circular shaped films, used for depth dose measurements on concave sources, were made by attaching a tube with a sharp edge to a drilling machine. This method worked best for HS films. MD55-2 film cutouts could be made by using the tube as a punch.

\subsubsection{Measurement methods for ophthalmic ruthenium sources}

The ruthenium sources were repositioned before each $60 \mathrm{~s}$ integration of the plastic scintillator.

The same types of radiochromic films and the same absorbed doses were used for measurements on ruthenium sources and the concave strontium sources (Table 5-4).

A diode detector was used for source uniformity measurements at the source surface in water. Depth dose measurements were performed with the diode using a depth dial indicator to determine the distance between detector and source surface. All uniformity measurements were performed in contact with the surface and perpendicular to the source surface.

Table 5-4 Film irradiation doses for measurements on all beta sources. Doses for depth dose measurements (PDD) are given at the surface, doses for the uniformity $(U)$ measurements are at $r=2$ $\mathrm{mm}$ (intravascular sources) and $z=0 \mathrm{~mm}$ (planar sources) and $z=3.4$ or $4.5 \mathrm{~mm}$ (concave strontium and ruthenium sources, respectively).

\begin{tabular}{|c|c|c|c|}
\hline & HD810 & MD55-2 & HS \\
\hline Intravascular sources & 100 Gy (U) & - & 30 Gy (U) \\
\hline $\begin{array}{c}\text { Planar ophthalmic } \\
\text { sources }\end{array}$ & $\begin{array}{c}400 \text { Gy (U) } \\
500 \text { Gy (PDD) }\end{array}$ & 80 Gy (PDD) & - \\
\hline $\begin{array}{c}\text { Concave ophthalmic } \\
\text { sources }\end{array}$ & - & 30 Gy (PDD) & $\begin{array}{c}30 \text { Gy (PDD) } \\
40 \text { Gy (U) }\end{array}$ \\
\hline
\end{tabular}

\subsubsection{Radiochromic film calibrations}

Because radiochromic film is tissue equivalent over a broad energy range, it was calibrated with a regular $6 \mathrm{MV}$ photon beam of a linear accelerator. All measurements were performed at the dose maximum of the PDD. A fit was performed according to Equation 5-2, in which $D_{w}$ is the absorbed dose to water and $O D$ is the optical density according to the red channel of the RGB (Red-Green-Blue) based TIF file. This channel has the strongest correlation with darkening of the film. The $O D$ was calculated using Equation 5-1. $T$ in this equation is transmittance, expressing the ratio of the pixel value at a given absorbed dose and the pixel value of film that was not irradiated.

$$
\begin{aligned}
& O D=-{ }^{10} \log (T) \\
& D_{w}=a+b_{1} O D+b_{2} O D^{2}+b_{3} O D^{3}
\end{aligned}
$$

The calibration curves of the radiochromic films are given in Figure 5-1 and Figure 5-2. The calibration curve is unique for each densitometer-film combination. Based on the specific shape of the curve a choice can be made for a $2^{\text {nd }}$ or $3^{\text {rd }}$ order polynomial. The uncertainty shown by the error bars is a combination of film non-uniformity and scanner noise. 


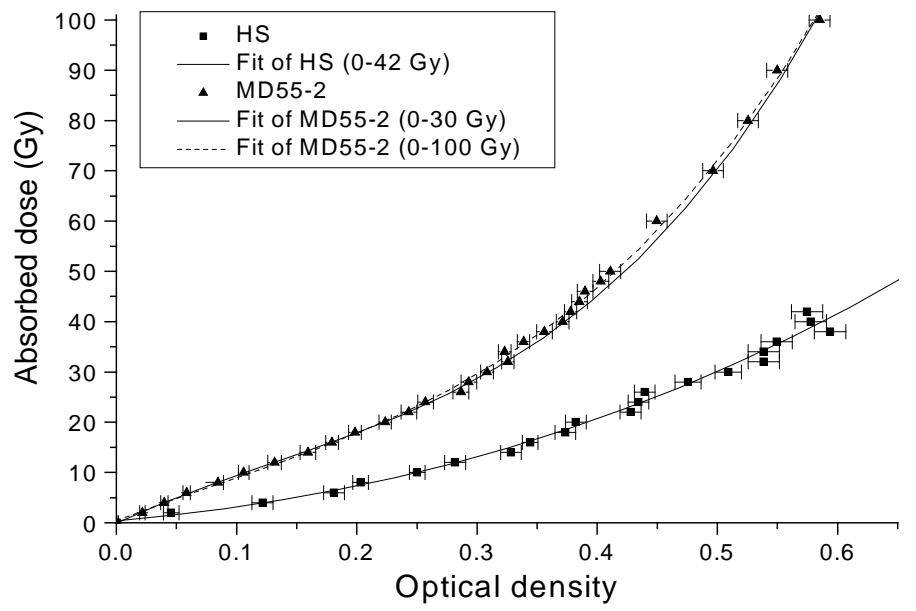

Figure 5-1 Absorbed dose as a function of optical density for HS and MD55-2 radiochromic film. Two different fits were used for MD55-2.

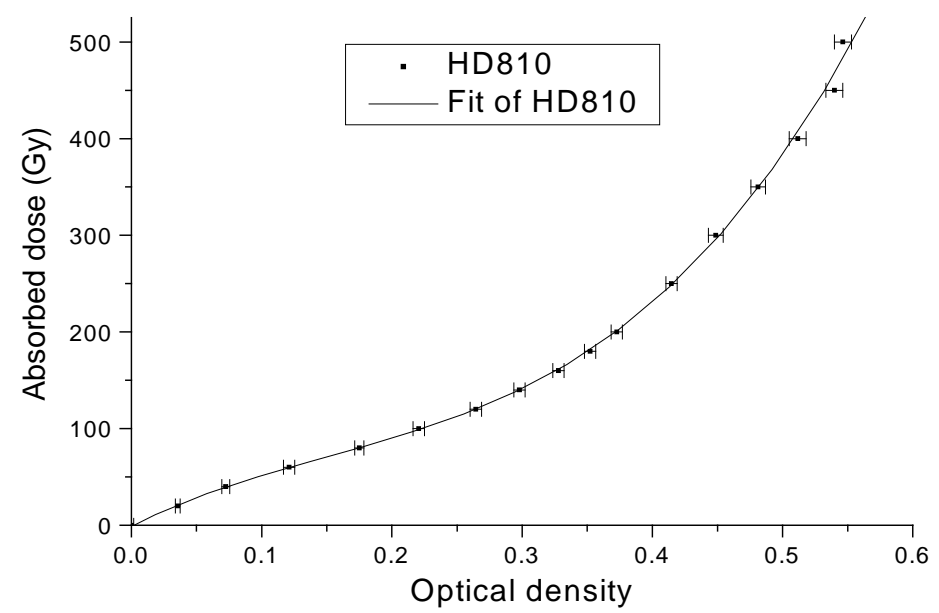

Figure 5-2 Absorbed dose as a function of optical density for HD810 radiochromic film.

\subsubsection{Film densitometry}

A flatbed transmission scanner was used for film densitometry (Microtek, see Section 3.3.4). This scanner was used in $3 \times 16$ bit mode with 12 bit color depth. The films were scanned at $300 \mathrm{dpi}$ (pixel size $0.08 \times 0.08 \mathrm{~mm}^{2}$ ). A spacing of about $1 \mathrm{~mm}$ between film and glass plate was used to prevent interference effects (Moiré artefacts/Newton rings).

\subsubsection{Film scan processing}

The TIF-files were processed using MATLAB (The MathWorks Inc., Matic, MA, USA). In the case of film measurements on line sources an averaging filter (median filter evaluating 10 pixel values) was applied to eliminate effects of dust particles on the film.

For the measurements on the ophthalmic sources a procedure was applied in which the pixels within a $1 \mathrm{~mm}$ area around each pixel were averaged. This averaging was carried out to eliminate the effects of small hot spots or noise in the evaluation of the source nonuniformity.

The procedures and definitions as described in Section 3.2 were used to evaluate source non-uniformity. 


\subsection{Estimation of measurement uncertainties}

During a number of introductory measurements measurement procedures were established as described in Appendix 10. Reproducibility of detector reading, influence of source and detector positioning, environmental influences and detector stability as verified through check source measurements were investigated. Results of these measurements are provided in the following sections. Reproducibility of the measurements, as function of source positioning, was taken from the series of on-site measurements as described in Section 5.4. In addition, the check source measurements accompanying these on-site measurements were used for Section 5.3.3.

\subsubsection{Positioning uncertainties}

The steep dose gradient of beta sources strongly dictates the importance of accurate source and detector positioning. The effects of positioning are shown in columns 1-3 of Table 5-5. The numbers in column 2 are the averaged standard deviations taken from the on-site measurements, described in Section 5.4. The measurements summarized in columns 2 to 4 , correspond to $\mathrm{n}$ measurements (as given in column 1) on $\mathrm{N}$ different sources. For every $60 \mathrm{~s}$ integration time, the source was reinserted in case of a measurement represented in column 2 , and the detector was repositioned for a measurement represented in column 3. Empty cells in column 3 indicate that it was not possible to change detector position without changing source position.

Phantom design and - if applicable - catheter geometry mainly determine positioning uncertainties. For plastic scintillator measurements on the ${ }^{32} \mathrm{P}$ source with the PTW Optidos phantom, large variations in reading were found, which seemed to be correlated to the curvature of the physics coupler. An experiment with a $360^{\circ}$ loop in the Guidant physics coupler gave differences in measured (60 s) values up to $9.3 \%$. Differences up to $6 \%$ were found with small changes in catheter shape even when the catheter was lined up straight. These differences probably were caused by the geometry of the phantom. The hole in which the source runs was wider than the source diameter. A (maximum) air gap of $0.14 \mathrm{~mm}$ between source and phantom was therefore possible. The potential difference in absorbed dose was estimated to be $7.5 \%$, based on a $1 / \mathrm{r}$ fall-off. The low average standard deviation (SD) of $0.5 \%$ in column 2 can be explained by the fixed shape of the physics coupler during a series of measurements.

Measurements on the Novoste sources with the IVB1000 well-type ionization chamber were performed with 3 source insertions and averaging over the 4 compass directions (total of 12 integrations). Effects of placement of the catheter in the chamber were investigated separately, but these were small compared with the effects of rotation of the chamber insert. The diameter of the hole in the phantom is an important factor in the uncertainty of the plastic scintillator and plane-parallel ionization chamber measurements on the SIA.20, because the diameter of this source type has changed during 20 or 25 years of production. The phantom was large enough for the newer sources, but too small for two of the old sources. For these sources an increased positioning uncertainty may be expected because centering the source had to be done without the phantom.

The plastic scintillator measurements on the ruthenium sources had a remarkable low standard deviation compared with those on the concave strontium sources. This difference was probably due to the more shallow dose fall-off of ruthenium than that of the concave strontium sources. Sources with a cutout had higher standard deviations (up to $3.5 \%$ for a CIA source). This higher standard deviation was due to the source positioning not being as stable as that for symmetrical sources, because the center of gravity was not on the central axis of the source. The high value for the positioning uncertainty of the diode measurements was caused by the fact that all measurements were performed in water. The distance could not be determined very precisely due to the use of a depth dial indicator. 
Table 5-5 Uncertainty due to positioning of source and detector, and stability of the detector for all types of sources.

\begin{tabular}{|c|c|c|c|c|c|c|}
\hline & (1) & $(2)$ & (3) & $(4)$ & (5) & $(6)$ \\
\hline & $\begin{array}{l}\text { \#measure- } \\
\text { ments } \\
\text { /series }\end{array}$ & $\begin{array}{c}\text { Source } \\
\text { positioning } \\
\text { (average SD) } \\
(n=1)\end{array}$ & $\begin{array}{c}\text { Detector } \\
\text { positioning } \\
\text { (average SD) } \\
(\mathrm{N}=1)\end{array}$ & $\begin{array}{c}\text { Measurement } \\
\text { reproducibility } \\
\text { (max. difference) } \\
(\mathrm{N}=1)\end{array}$ & $\begin{array}{l}\text { Detector } \\
\text { stability }\end{array}$ & $\begin{array}{c}\text { Estimated } \\
\text { combined } \\
\text { standard } \\
\text { uncertainty }^{\dagger}(1 \sigma)\end{array}$ \\
\hline $\begin{array}{l}\text { HDR1000+ } \\
\text { WIC } \\
\text { with }{ }^{32} P \\
\text { Guidant }\end{array}$ & $6 \times 60 \mathrm{~s}$ & $\begin{array}{l}0.06 \% \\
(\mathrm{~N}=12)\end{array}$ & - & $0.2 \%(\mathrm{n}=2)$ & & $0.2 \%$ \\
\hline $\begin{array}{c}\text { IVB1000 WIC } \\
\text { with }{ }^{90} \mathrm{Sr}{ }^{90} \mathrm{Y} \\
\text { Novoste }\end{array}$ & $12 \times 60 \mathrm{~s}^{\ddagger}$ & $\begin{array}{c}0.6 \% \\
(\mathrm{~N}=11)\end{array}$ & - & $\begin{array}{c}0.8 \%(\mathrm{n}=2,3.5 \mathrm{~F}) \\
0.7 \%(\mathrm{n}=2,5 \mathrm{~F})\end{array}$ & & $0.5 \%$ \\
\hline $\begin{array}{c}\text { Optidos PS } \\
\text { with }{ }^{32} P \\
\text { Guidant }\end{array}$ & $5 \times 60 \mathrm{~s}^{\S}$ & $\begin{array}{c}0.5 \% \\
(\mathrm{~N}=12)\end{array}$ & $0.2 \%(n=1)$ & $4 \%(n=2)$ & $3 \%$ & $5 \%^{t \dagger}$ \\
\hline $\begin{array}{c}\text { Optidos PS } \\
\text { with }{ }^{90} \mathrm{Sr}^{90} \mathrm{Y} \\
\text { Novoste }\end{array}$ & $5 \times 60 \mathrm{~s}$ & $\begin{array}{c}1 \%(3.5 \mathrm{~F}, \mathrm{~N}=4) \\
2 \%(5 \mathrm{~F}, \mathrm{~N}=3)\end{array}$ & $0.2 \%(n=1)$ & $\begin{array}{c}5.6 \%(\mathrm{n}=4,3.5 \mathrm{~F}) \\
2.0 \%(\mathrm{n}=2,5 \mathrm{~F})\end{array}$ & $3 \%$ & $3 \%$ \\
\hline $\begin{array}{l}\text { Optidos PS } \\
\text { with }{ }^{90} \mathrm{Sr}^{90} \mathrm{Y} \\
\text { planar }\end{array}$ & $5 \times 60 s^{\S}$ & $\begin{array}{l}0.7 \% \\
(\mathrm{~N}=5)\end{array}$ & $1 \%(n=1)$ & $2.4 \%(n=5)$ & $3 \%$ & $3 \%$ \\
\hline $\begin{array}{c}\text { Optidos PS } \\
\text { with }{ }^{90} \mathrm{Sr}^{\rho 0} \mathrm{Y} \\
\text { concave }\end{array}$ & $5 \times 60 \mathrm{~s}$ & $\begin{array}{l}0.5 \% \\
(\mathrm{~N}=5)\end{array}$ & $0.6 \%(n=1)$ & $0.5 \%(n=2)$ & $3 \%$ & $3 \%$ \\
\hline $\begin{array}{c}\text { Optidos PS } \\
\text { With } \\
{ }^{106} \mathrm{Ru}^{106} \mathrm{Rh} \\
\text { BEBIG (only } \\
\text { for sym. } \\
\text { sources) }\end{array}$ & $5 \times 60 \mathrm{~s}$ & $\begin{array}{l}0.1 \% \\
(\mathrm{~N}=4)\end{array}$ & $0.5 \%(n=1)$ & - & $3 \%$ & $3 \%$ \\
\hline $\begin{array}{c}\text { Markus PPIC } \\
\text { with }{ }^{90} \mathrm{Sr}{ }^{90} \mathrm{Y} \\
\text { planar }\end{array}$ & $5 \times 60 \mathrm{~s}^{\S}$ & $\begin{array}{l}0.8 \% \\
(\mathrm{~N}=5)\end{array}$ & - & $1.5 \%(n=6)$ & $<1.1 \%$ & $\begin{array}{c}2 \% \\
\text { (1 source) }\end{array}$ \\
\hline $\begin{array}{l}\text { Scanditronix } \\
\text { diode } \\
\text { with } \\
{ }^{106} \mathrm{Ru} /{ }^{106} R h \\
B E B I G\end{array}$ & $\begin{array}{c}\text { no series } \\
\text { measured } \\
(1 \times 60-180 \mathrm{~s})\end{array}$ & - & $\begin{array}{c}<5 \% \\
\text { (estimated } \\
\text { at } 2 \mathrm{~mm} \text {, } \\
\text { no SD) }\end{array}$ & - & $<2 \%$ & $4 \%$ \\
\hline $\begin{array}{c}\text { Radiochromic } \\
\text { film }\end{array}$ & & & & & & $\begin{array}{c}2 \% \text { (HD810) } \\
3 \%(\mathrm{HS}) \\
3 \% \text { (MD55-2) }\end{array}$ \\
\hline
\end{tabular}

$N$ is the number of sources; $n$ is the number of independent experiments on one source (as given in column 1, see Section 5.3.2). WIC: well-type ionization chamber; PS: plastic scintillator; PPIC: planeparallel ionization chamber.

\footnotetext{
* Repeated source insertions.

${ }^{\dagger}$ Excluding uncertainty introduced by absolute calibration.

¥ Averaging over 3 source insertions and 4 compass directions.

${ }_{\star \star}^{\S}$ More measurements were performed in the case of higher standard deviations.

${ }^{* *}$ See Figure 5-3 for more details; 90\% (27/30) of the experiments have a difference in check source reading below $3 \%$.

$\mathrm{t \dagger}^{\mathrm{T}}$ The largest contribution to this uncertainty was due to the PTW phantom itself.
} 


\subsubsection{Measurement reproducibility}

Measurements were performed in order to test the reproducibility of detector readings. To this end, the configuration for the measurements was completely rebuilt in order to take as many factors of uncertainty into account as possible. The time between measurements varied from hours to months. Maximum (percent) differences between measurements performed on a single source by a single observer are given in column 4 of Table 5-5. Measurement series are listed in column 1.

\subsubsection{Detector stability}

Estimates of the stability of different detectors are given in column 5 of Table 5-5. For plastic scintillator, diode and plane-parallel ionization chamber these values are based on check source measurements. For the diode the estimated stability is below $2 \%$. Because the check source measurements did not take place in a solid phantom, but in-air, the $2 \%$ value can be largely attributed to positioning uncertainty. For the plane-parallel ionization chamber the differences in response were below $1.1 \%$. Although this value is somewhat high for this detector, the difference is acceptable for current applications. For the plastic scintillator larger differences were noticed. With the plastic scintillator differences of $1-2 \%$ were frequently found between the two check source measurements before and after a measurement series of no more than 40 minutes. PTW specifies a maximum drift of $1 \%$ in 40 minutes. The two largest differences were $21.6 \%$ and $9.4 \%$. These differences were noticed after analysis of the on-site measurements. It was an incidental value of the check source measurement, as can be seen from the statistics of 30 measurements shown in Figure 5-3. Three out of 30 measurements had a difference larger than 3\%.

The sensitivity of the plastic scintillator decreased with $~ 35 \%$ during 8 months, therefore justifying the correction for this sensitivity decrease that was (automatically) done by means of the check source measurements. The estimated absorbed dose of the detector was in the order of kiloGrays during the period of use.

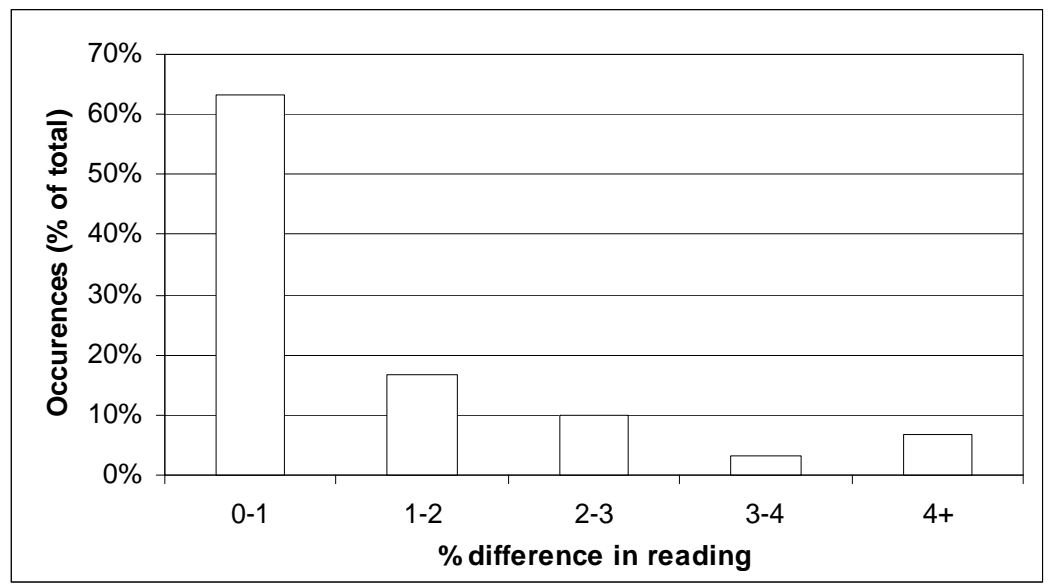

Figure 5-3 Percentage change in plastic scintillator sensitivity (frequency in \% of total) during a 40 minute period according to check source measurement.

\subsubsection{Environmental dependence}

Detector warm-up effects were eliminated by taking into account a warming-up period of at least 30 minutes before initiating the first measurement. This applied to all measurements except those with the diode and radiochromic film. Temperature and pressure read-out errors were estimated to contribute up to $0.03 \%$ to the uncertainty in the correction of temperature and pressure of the ionization chamber. 
Measurements with the Optidos plastic scintillator were not affected by slow environmental changes, because the check source corrections took these slow variations in detector sensitivity into account.

Users of radiochromic film should be aware of light and temperature effects [42]. The film color darkens during the first days after irradiation. At $37^{\circ} \mathrm{C}$ color stabilization is accelerated by a factor of up to 5 compared with $22^{\circ} \mathrm{C}$ [75]. There are no reports that temperatures below $37^{\circ} \mathrm{C}$ have effects on films. The manufacturer advises storage below $25^{\circ} \mathrm{C}$. Effects of temperature exposure between $25-30^{\circ} \mathrm{C}$ are estimated to be no more than a few percent when a 24 hours post-irradiation time is used.

\subsubsection{Other dependencies}

For the well-type ionization chambers it was investigated whether an adjacent wall (in this case a small lead castle) affected the amount of backscatter, but the measurements did not show an increase in signal.

\subsubsection{Estimated uncertainty}

Based on the measurements mentioned above, an estimate was made of the total measurement uncertainty. These uncertainty values are given in the last column of Table 5-5 and are also valid for the inter-institute measurements as presented in the following section. The uncertainty in an absolute calibration such as a $2 \sigma$ uncertainty of $15 \%$ as (often) specified by NIST, however, is not included. Derived quantities used in Section 5.4 are given in Table 5-6. The measurement uncertainties as given here are indicated in the graphs of the following section with the $\Delta$ symbol.

For the radiochromic film measurements no separate investigation on measurement uncertainties was performed. Based on the experience gained during the on-site measurements, it was estimated that the relative standard uncertainties are between $2-3 \%$ $(1 \sigma)$. These values include uncertainties caused by film non-uniformity, calibration and scanner readout. With double exposure techniques it is possible to lower this uncertainty.

Table 5-6 Measurement standard uncertainties (1 $\sigma)$ for all parameters of interest in this chapter.

\begin{tabular}{|c|c|c|c|c|}
\hline & $\dot{D}\left(r_{0}, z_{0}\right)$ & $\dot{D}\left(r_{0}\right)$ & $\begin{array}{l}\boldsymbol{U}\left(\boldsymbol{U}_{F}, \boldsymbol{U}_{A S}, \boldsymbol{U}_{I C R U}\right) \\
\text { (if applicable) }\end{array}$ & ASL \\
\hline Guidant & PS: $5 \%$ & $\begin{array}{l}\text { WIC: } 0.2 \% \\
\text { PS: } 5 \%{ }^{*}\end{array}$ & $\begin{array}{l}\text { HD810: } 3 \% \\
\text { HS: } 4 \% \\
\text { PS: } 5 \%\end{array}$ & film: $0.2 \mathrm{~mm}$ \\
\hline Novoste & PS: $3 \%$ & $\begin{array}{l}\text { WIC: } 0.5 \% \\
\text { PS: } 3 \%{ }^{*}\end{array}$ & $\begin{array}{l}\text { HD810: } 3 \% \\
\text { HS: } 4 \% \\
\text { PS: }>3 \%\end{array}$ & film: $0.2 \mathrm{~mm}$ \\
\hline Amersham SIA.20 & $\begin{array}{l}\text { PS: } 3 \% \\
\text { PPIC: } 2 \%\end{array}$ & - & HD810: 3\% & - \\
\hline Amersham SIA.6 & PS: 3\% & - & HS: 4\% & - \\
\hline BEBIG & $\begin{array}{l}\text { PS: } 3 \% \\
\text { Diode: } 4 \%\end{array}$ & - & $\begin{array}{l}\text { Diode: }>3 \%^{\dagger} \\
\text { HS: } 4 \%{ }^{\ddagger}\end{array}$ & - \\
\hline
\end{tabular}

\footnotetext{
Under the assumption that the uncertainty in measuring will not differ much from $\dot{D}\left(r_{0}, z_{0}\right)$. This was not investigated separately. $\dot{D}\left(r_{0}\right)$ is deduced from the average of 7 (Guidant), 5 (Novoste $60 \mathrm{~mm}$ ) or 3 (Novoste $40 \mathrm{~mm}$ ) integrations of $60 \mathrm{~s}$ along the source axis.

${ }^{\dagger}$ A minimum value can only be estimated, as no full sampling of the source was carried out.

${ }^{\ddagger}$ Artifacts that may occur for the BEBIG sources due to the edges of the film are excluded.
} 


\subsection{Results of inter-institute measurements}

Measurements with the detectors given in Section 5.1.2 were performed throughout the Netherlands and Belgium. The purpose of these on-site measurements was:

1) testing of measurement methods on multiple clinical sources,

2) demonstrating potential measurement methods to users of beta sources, and

3) gathering information on the consistency of source calibrations by manufacturers and on the uniformity of clinical sources.

The figures of strength measurements show the value as specified by the manufacturer and corrected for source decay, versus the value as measured with the detector used in the survey. Absolute calibration of the detectors was not possible, as the NMi primary standard was not fully validated at the time of writing. In order to compare the strength measurements with each other, the assumption was made that the average difference of all measurements on one source type with one detector was zero*. Although this normalization may hide systematic strength deviations of the manufacturers' standard with a primary standard, it does quantify the consistency of the source calibrations by the manufacturer as compared with the measurements performed in this survey. A low standard deviation suggests that manufacturers' calibration and survey measurement are consistent with each other; a high standard deviation implies that the manufacturer and/or survey measurements contain large variations.

The source strength specified by the manufacturer was derived from the certificate by applying a correction for source decay and, where necessary, depth dose. The decay correction for the time difference $\left(t-t_{0}\right)$ was performed according to Equation 5 -3. Half-lives $T_{1 / 2}$ are given in Appendix 1.

$\dot{D}_{w}(t)=\dot{D}_{w}\left(t_{0}\right) e^{-\left(t-t_{0}\right) \ln 2 / T_{1 / 2}}$

\subsubsection{Intravascular sources}

The source strength of the intravascular sources was determined with a well-type ionization chamber and a plastic scintillator. Relative differences between measured dose rate and decay corrected certificate values are displayed in Figure 5-4 for the Guidant ${ }^{32} \mathrm{P}$ sources and in Figure 5-5 for the Novoste ${ }^{90} \mathrm{Sr} /{ }^{90} \mathrm{Y}$ sources. In both figures a distinction is made between $\dot{D}\left(r_{0}, \mathrm{z}_{0}\right)$ and $\dot{D}\left(r_{0}\right)$ for the plastic scintillator measurements (the rate sign $\bullet$ is dropped in the figures for practical reasons). The measurements were performed on different sources at different institutes during a period of three months. Consistent identification of sources by number is used for presentation of strength and non-uniformity measurements.

For calculation of a margin of 2.5 or $3 \mathrm{~mm}$, as described in Section 3.2.1, was applied. $\dot{D}\left(r_{0}\right)$ was determined using the average of 8 integrations with $2 \mathrm{~mm}$ spacing for the Guidant sources, and either 3 or 5 integrations (depending on source length) with $10 \mathrm{~mm}$ spacing for the Novoste sources. Novoste sources 1-4 (length of $40 \mathrm{~mm}$ ) and 5-6 $(60 \mathrm{~mm})$ were of the 3.5F type; sources 7-9 $(40 \mathrm{~mm})$ and 10-12 $(60 \mathrm{~mm})$ were of the older $5 \mathrm{~F}$ type. For the welltype ionization chamber measurements on those sources a separate normalization was performed for all four subgroups, as both length and type of source and catheter may affect the magnitude of the signal.

\footnotetext{
* The only exception was made for the strength measurements on the planar strontium sources, because measurements on two of the sources gave deviating results.
} 


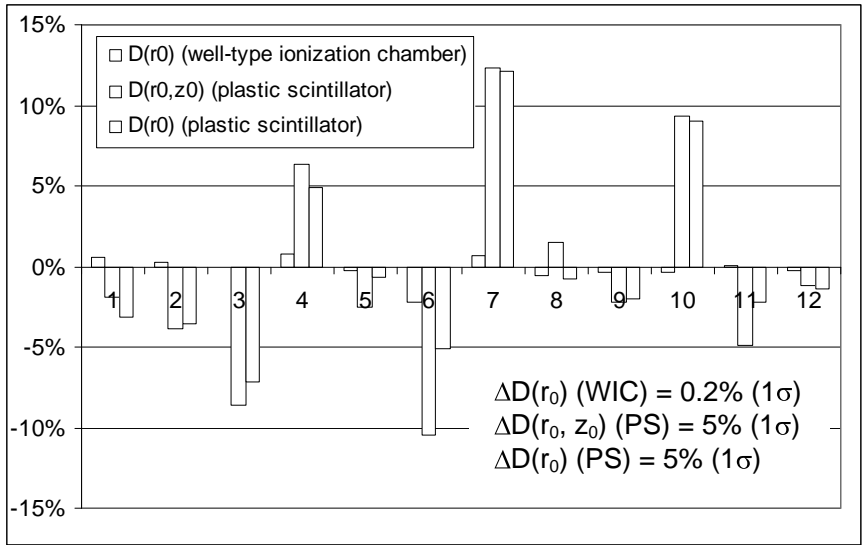

Figure 5-4 Relative difference (D-S) in source strength between specification $(S)$ and detector measurement $(D)$ for Guidant ${ }^{32} P$ sources (data normalized to $0 \%$ ).

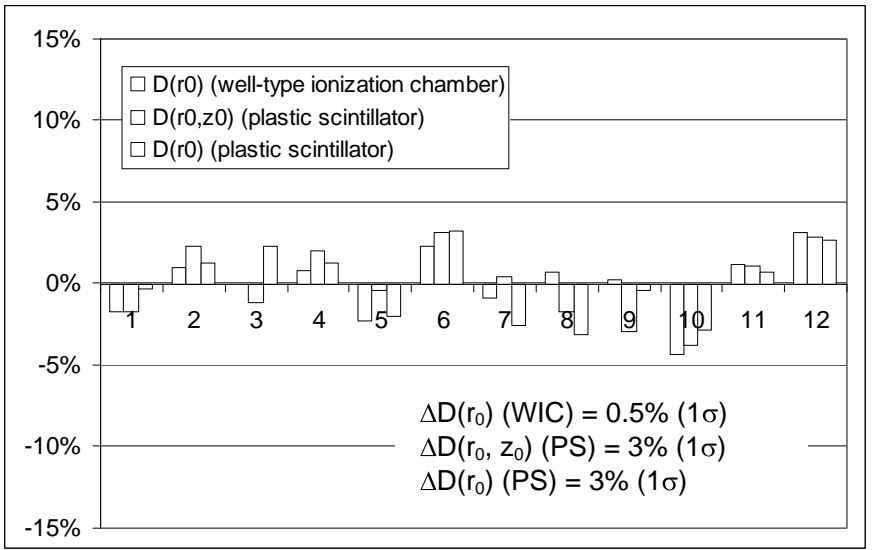

Figure 5-5 Relative difference (D-S) in source strength between specification $(S)$ and detector measurement (D) for Novoste ${ }^{90} \mathrm{Sr}^{90} \mathrm{Y}$ sources (data normalized to $0 \%$ ).

Three of the well-type ionization chamber measurements on the Novoste sources $(1,2$ and 5 ) needed an additional correction of $\sim 1 \%$ because the insert was glued after it broke at the beginning of the measurement series. The standard deviations of the differences between measurements and the certificate values are given in Table 5-7. These numbers indicate the (relative) consistency between the calibration of the manufacturer and the present measurement results. Offsets are not revealed due to the relative character of the measurements. Maximum differences in between plastic scintillator and well-type ionization chamber measurements were $11.5 \%$ for the Guidant sources (source 7 ) and $3.8 \%$ for the Novoste sources (source 8).

Table 5-7 Standard deviations of relative difference (D-S) in source strength between specification $(S)$ and detector measurements $(D)$ for different detectors and source types.

\begin{tabular}{|l|l|l|l|}
\hline Detector & $\begin{array}{l}\text { Guidant } \\
\text { sources (SD) }\end{array}$ & $\begin{array}{l}\text { Novoste sources (SD) } \\
(\mathbf{3 . 5 F})\end{array}$ & $\begin{array}{l}\text { Novoste sources (SD) } \\
(\mathbf{5 F})\end{array}$ \\
\hline $\begin{array}{l}\text { Well-type ionization } \\
\text { chamber }\end{array}$ & $0.8 \%(1 \sigma, \mathrm{N}=12)$ & $2.0 \%(1 \sigma, \mathrm{N}=5)$ & $3.0 \%(1 \sigma, \mathrm{N}=6)$ \\
\hline Plastic scintillator $\left(\mathbf{r}_{0}, \mathbf{z}_{0}\right)$ & $7.2 \%(1 \sigma, \mathrm{N}=12)$ & $2.0 \%(1 \sigma, \mathrm{N}=6)$ & $2.6 \%(1 \sigma, \mathrm{N}=6)$ \\
\hline Plastic scintillator $\left(\mathbf{r}_{0}\right)$ & $6.0 \%(1 \sigma, \mathrm{N}=12)$ & $1.9 \%(1 \sigma, \mathrm{N}=6)$ & $2.3 \%(1 \sigma, \mathrm{N}=6)$ \\
\hline
\end{tabular}

The spread of the difference between well-type ionization chamber measurements and certificate was small for the Guidant sources as the same type of detector was used by the manufacturer. For the plastic scintillator measurements this spread was larger. Three factors may have contributed to the magnitude of this spread:

- Radial positioning uncertainty of the source in the PTW phantom (effects up to $9 \%$ possible, see Section 5.3.1). This is expected to give the largest contribution to the spread.

- Change in plastic scintillator sensitivity (up to $4.6 \%$ during this series, see Section 5.3.3).

- Difference between a point and a volume measurement.

The third factor may be of importance because Guidant's in-house calibration is performed

with a well-type ionization chamber and therefore gives a source strength $\dot{D}\left(r_{0}\right)$ based on the total activity contained within the source, assuming a uniform source. The absorbed dose rate as measured with a plastic scintillator in $\left(r_{0}, z_{0}\right)=(2,0) \mathrm{mm}$, however, is a different parameter. Some of the Guidant sources are non-uniform, having a dip of a few \% in the middle and therefore a difference between plastic scintillator and well-type ionization chamber measurement may be expected. This effect is illustrated in the graphs by the difference between $\dot{D}\left(r_{0}\right)$ and $\dot{D}\left(r_{0}, \mathrm{z}_{0}\right)$ for the plastic scintillator measurements. It is most 
profound for the measurements on the Guidant sources $\left(\mathrm{SD}=6.0 \%\right.$ for $\dot{D}\left(r_{0}\right)$ vs. SD $=7.2 \%$ for $\left.\dot{D}\left(r_{0}, \mathrm{z}_{0}\right)\right)$.

The standard deviation of the well-type ionization chamber and plastic scintillator measurements for the Novoste sources was limited (all within $\pm 5 \%$ ) and the standard deviation was of the same order of magnitude (up to $3 \%$ ). The lower standard deviation of the plastic scintillator measurements on the Novoste compared with the Guidant sources, can be explained by the better phantom geometry.
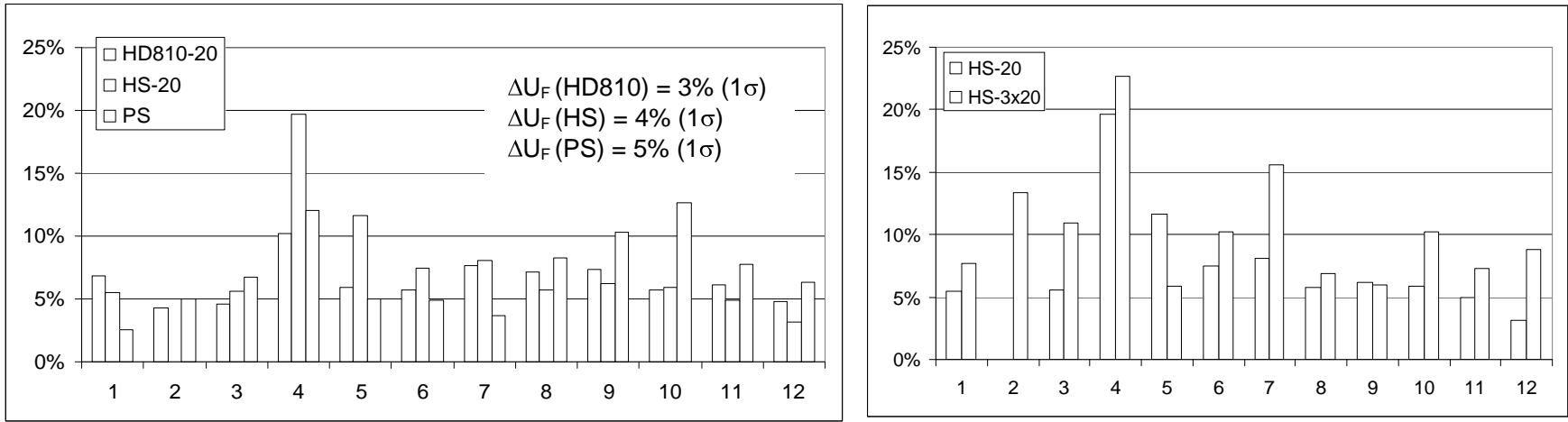

Figure 5-6 Source non-uniformity $U_{F}$ for Guidant ${ }^{32} P$ sources as measured with plastic scintillator (PS) and radiochromic film (HD810, $H S)$.

The non-uniformity $U_{F}$ of the intravascular sources was determined as described in Section 3.2.1 (extremes compared with average value, margins $M_{P}$ and $M_{S r}$ ). The non-uniformity of all 12 clinical ${ }^{32} \mathrm{P}$ Guidant sources is given in Figure 5-6 for the two types of radiochromic film (HD810, HS) and the plastic scintillator. An example of one of the source profiles (\#12) is given in Figure 5-8. The contribution of stepping the source to non-uniformity is shown in Figure 5-7. The same HS film measurement is given in Figure 5-6.

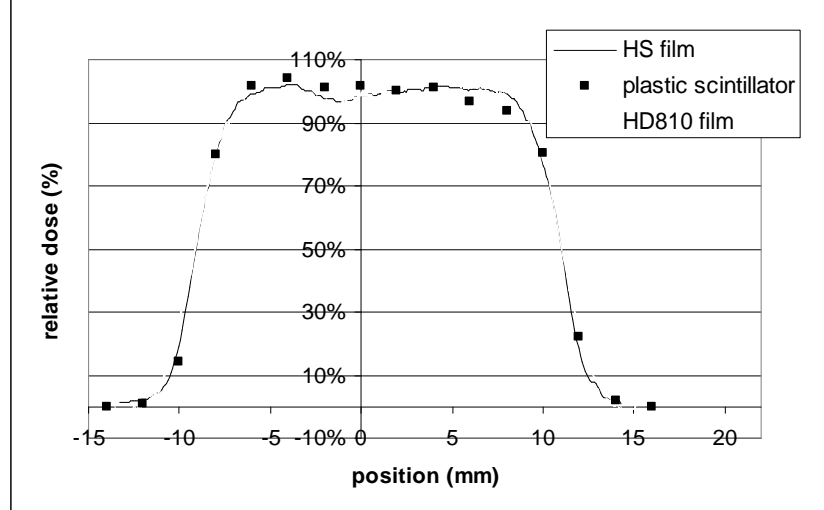

Figure 5-8 Source profile of one Guidant ${ }^{32} P$ source (\#12). Measurement with HD810, HS and plastic scintillator.
Figure 5-7 Source non-uniformity $U_{F}$ for Guidant ${ }^{32} P$ sources as measured with HS radiochromic film. Both single source and 3 source positions are displayed (2 steps of 20 $\mathrm{mm})$.

The non-uniformity $U_{F}$ determined with HD810 is lower than that for the plastic scintillator and HS film measurements, as is illustrated in Table 5-8. This is probably due to local variations in the thickness of the sensitive layer of HS that do not occur with HD810. The plastic scintillator measurements were not always consistent with the film measurements; additional errors may have been introduced by the size of the bore through which the source runs. Non- 
uniformity of the HS measurements increased with stepping of the sources (from $7.0 \%$ to $10.6 \%$, on average). Part of this increase can be explained by slight variations in film sensitivity that were more pronounced for (some of the) long films. For some of these films there was a gradual change in the height of the plateau. The stepping itself also contributed to the larger non-uniformity as can be seen in Figure 5-9, where repetition of the source profile can be recognized.

The non-uniformity $U_{F}$ of all 12 clinical ${ }^{90} \mathrm{Sr} /{ }^{90} \mathrm{Y}$ Novoste sources is given in Figure 5-10. The source profile of source \#6 is shown in Figure 5-11 as an example.

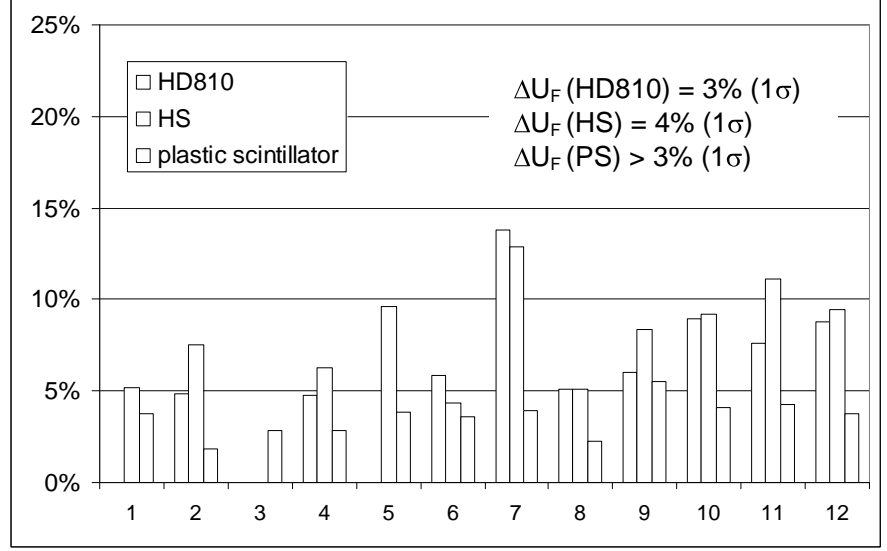

Figure 5-10 Source non-uniformity $U_{F}$ of the ${ }^{90} \mathrm{Sr}^{90} \mathrm{Y}$ sources as measured with plastic scintillator and radiochromic film (HD810, HS). Source numbers are given in Section 5.4.1.

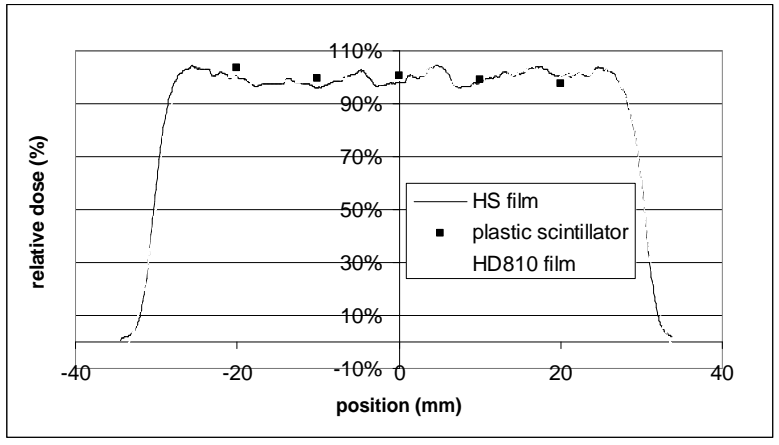

Figure 5-11 Source profile of one $60 \mathrm{~mm}$ $3.5 F^{90} \mathrm{Sr}^{90} \mathrm{Y}$ source (source \#6), Measurement with HD810, HS and plastic scintillator.

The average source non-uniformity of the $3.5 \mathrm{~F}$ sources was a few percent better than that of the 5F sources, as can be seen in Table 5-8. The better uniformity can be explained by the fact that the $3.5 \mathrm{~F}$ sources are jacketed and the 5F sources consist of loose seeds.

The non-uniformity as determined by plastic scintillator measurements made use of data at $z$ $=0, \pm 10, \pm 20 \mathrm{~mm}$. For the $40 \mathrm{~mm}$ sources, however, only the measured values at $z=0$ and $\pm 10 \mathrm{~mm}$ were used. Average non-uniformity of plastic scintillator measurements was lower than that of radiochromic film measurements, simply because only part of the source length was evaluated. Again, with HD810 film a lower non-uniformity was achieved than with HS, due to the variations in film sensitive layer thickness mentioned earlier.

Table 5-8 Average source non-uniformity $U_{F}$ as determined with plastic scintillator and radiochromic film (HS, HD810).

\begin{tabular}{|l|l|l|l|}
\hline Detector & $\begin{array}{l}\text { Guidant } \\
\text { sources }\end{array}$ & $\begin{array}{l}\text { Novoste sources } \\
(\mathbf{3 . 5 F})\end{array}$ & $\begin{array}{l}\text { Novoste sources } \\
\mathbf{( 5 F})\end{array}$ \\
\hline Plastic scintillator & $7.1 \%$ & $3.1 \%$ & $4.0 \%$ \\
\hline HD810 radiochromic film & $5.7 \%$ & $5.0 \%$ & $7.5 \%$ \\
\hline HS radiochromic film & $7.0 \%$ & $5.3 \%$ & $8.9 \%$ \\
\hline HS radiochromic film $(\mathbf{3 x 2 0} \mathbf{~ m m})$ & $10.6 \%$ & & \\
\hline
\end{tabular}

The active source length of the line sources was determined from the radiochromic film data according to the definition in Section 3.2.1. The deviation from the nominal source length (NSL $=20 \mathrm{~mm}$ for Guidant sources and 40 or $60 \mathrm{~mm}$ for the Novoste sources) is shown in Figure 5-12 and Figure 5-13. The data set is incomplete due to technical problems during the measurements (for example no film measurement was performed with HS on Guidant source 2). The average ASL values are close to the nominal values. 


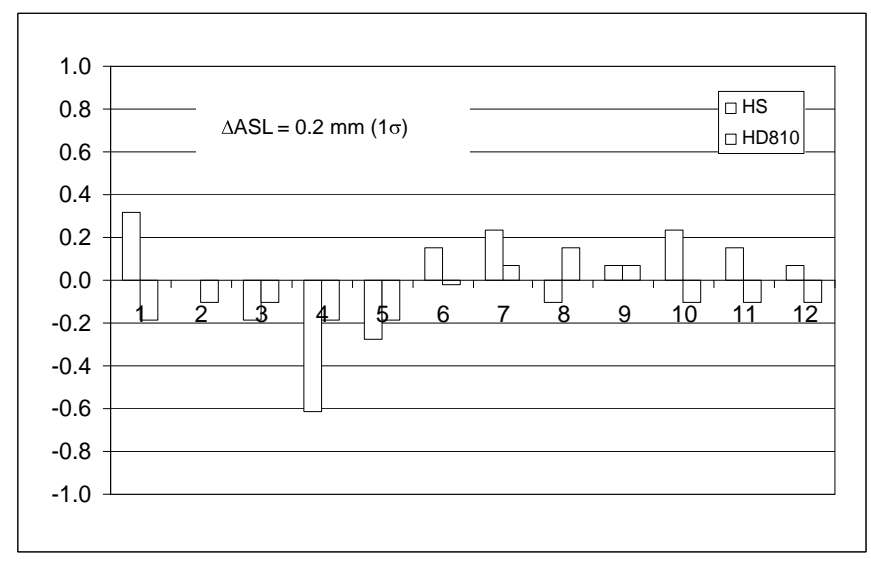

Figure 5-12 Difference (ASL-NSL) in $\mathrm{mm}$ between active and nominal source length for the Guidant $20 \mathrm{~mm}$ sources as determined with HS and HD810 film measurements.

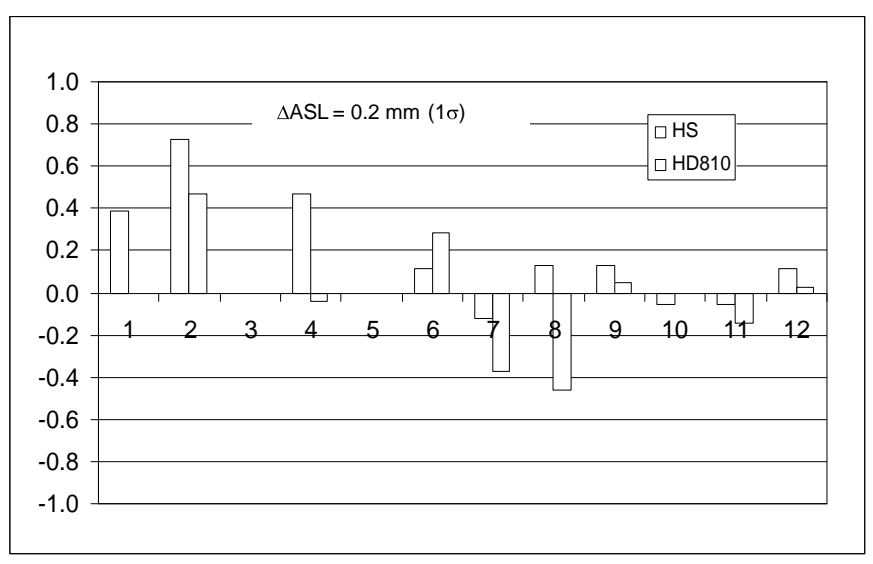

Figure 5-13 Difference (ASL-NSL) in $\mathrm{mm}$ between active and nominal source length for the Novoste 40 and $60 \mathrm{~mm}$ sources as determined with HS and HD810 film measurements.

In the previous section it was concluded that HS measurements were less reliable than HD810 measurements. Here, the largest deviations in active source length were also found with HS film (for example Guidant source 4). The maximum deviation in ASL with HD810 was less than $0.5 \mathrm{~mm}$ of the nominal source length.

\subsubsection{Ophthalmic strontium sources}

The source strength of the Amersham ${ }^{90} \mathrm{Sr} /{ }^{90} \mathrm{Y}$ sources was measured at $z_{0}=2 \mathrm{~mm}$ using a plastic scintillator. Plane-parallel ionization chamber measurements were performed at a different distance because of the thickness of the protective cover. Relative differences between the present measurements and the manufacturer's certificate are given in Figure 5-14 for the planar sources and in Figure 5-15 for the concave sources (those for the planar sources were not normalized to zero).

Planar sources P4 and P5 were too large for the phantom and measurements were performed without the phantom in order to be able to center the applicator. The diameter of source P3 was smaller than that of P1 and P2, so that centering of the source in the phantom was less accurate. For that reason a second measurement was performed, presented here, in which centering was performed more carefully. For planar source P5 no certificate value was available and a value determined at the institute through large electrode extrapolation chamber measurements in the 1980s was used. At the time of delivery an additional resin layer was added to the source surface to lower the dose rate. The large decrease $(21.6 \%)$ in plastic scintillator sensitivity as described in Section 5.3.3 took place during the measurement on source P4. No explanation for this change in sensitivity has been found. Concave sources $\mathrm{C} 3$ and $\mathrm{C} 4$ had larger radii of curvature than the standard $10 \mathrm{~mm}$, so that the spherical phantom did not fit the source surface during plastic scintillator measurements. For these measurements a phantom with a larger radius of curvature of $12 \mathrm{~mm}$ was used, although the actual radii of curvature of sources C3 and C4 were larger. 


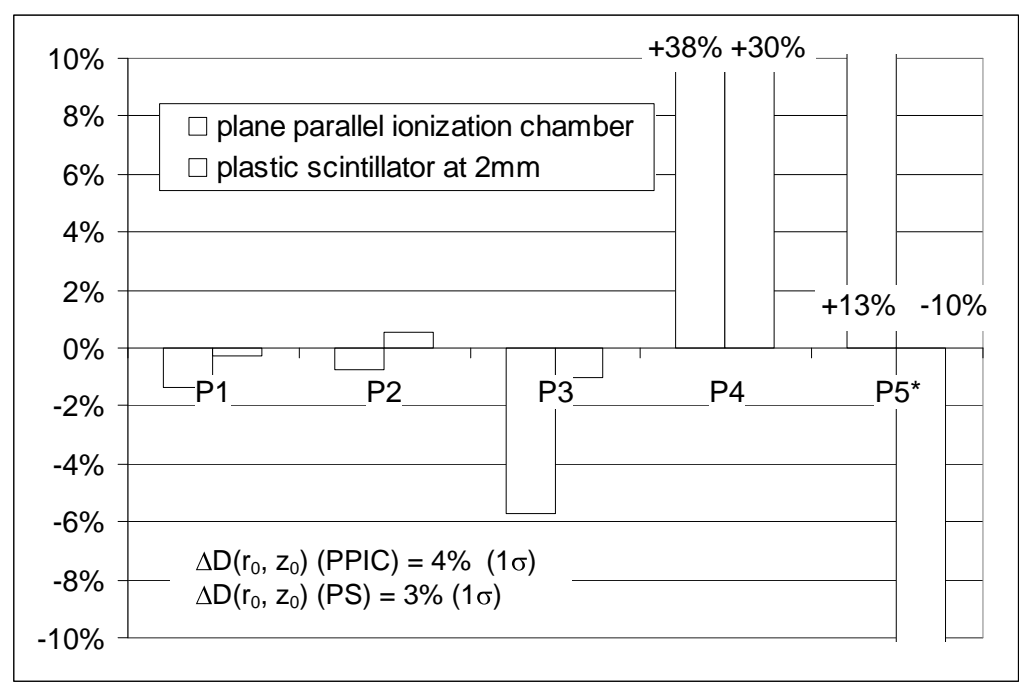

Figure 5-14 Relative difference (D-S) in source strength between specification (S) and detector (plastic scintillator or plane-parallel ionization chamber measurements (D) for planar Amersham ${ }^{90} \mathrm{Sr}{ }^{90} \mathrm{Y}$ sources).

* (P5): no certificate value available. $P 4$ and $P 5$ have larger average radii.

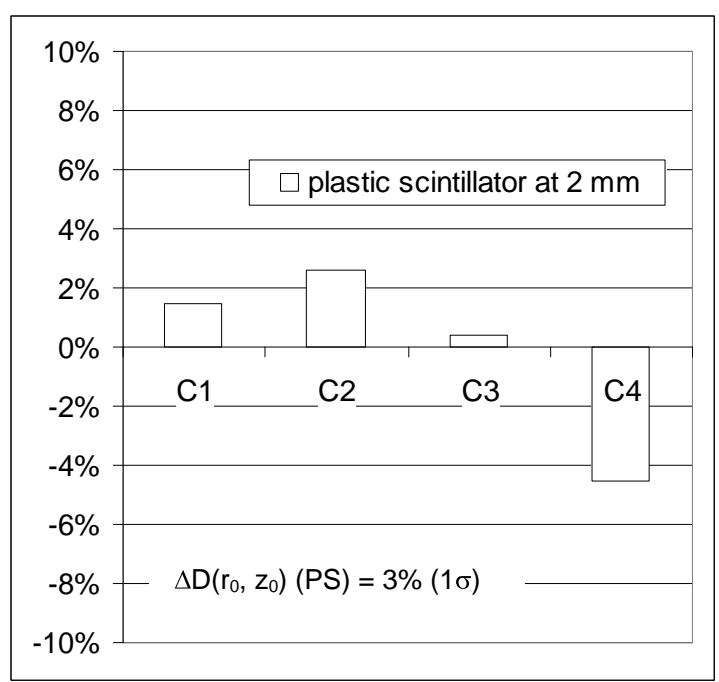

Figure 5-15 Relative difference $(P-S)$ in source strength between specification (S) and plastic scintillator $(P)$ measurements for concave Amersham ${ }^{90} \mathrm{Sr}^{90} \mathrm{Y}$ sources as measured at $2 \mathrm{~mm}$ (data normalized to $0 \%)$.

Figure 5-14 shows an agreement of measurements on P1-P3 within 5\%. These sources had similar sizes and activity distributions. The other two sources (P4 and P5) had different activity distributions and average radii, as can be seen in Figure 5-18a and b. The difference between these two dose distributions affects the number of ionizations in the plane-parallel chamber volume with a diameter of $5 \mathrm{~mm}$. Therefore sources P4 and P5 should be considered as different source types. The change in plastic scintillator sensitivity will have affected the results of the measurements on source P4. For P5 no certificate was available. Because of these reasons the agreement between measurements on P1-P3 and P4-P5 was small.

The spread of plastic scintillator measurements on the concave sources was small with a standard deviation of $3.7 \%$, two different radii of curvature included. It was not known whether sources C3 and C4 were calibrated by Amersham using a solid (and fitting) phantom. The effect of an air gap resulting from the deviating source curvature is probably small at $(z, r)=(2,0) \mathrm{mm}$ as the largest contribution to the signal is due to activity close to the axis. The size of this air gap is determined by the cosine of the angle between source and phantom and therefore not very large. 
Source non-uniformity of the Amersham ${ }^{90} \mathrm{Sr} /{ }^{90} \mathrm{Y}$ sources was studied with radiochromic film. Calculated non-uniformity values are shown in Figure 5-16 for the planar sources and in Figure 5-17 for the concave sources. The calculation was performed with the concept as introduced by the ICRU $\left(U_{\text {ICRU }}\right)$ and the alternative concept as introduced in Section 3.2.2 $\left(U_{F}\right)$. Measurements were performed in the plane perpendicular to the $z$-axis at the source surface for the planar sources and at $3.4 \mathrm{~mm}$ in solid water for the concave SIA.6 sources. For the concave sources only the non-uniformity of the in-phantom measurements is displayed. Because of the deviating applicator curvature, no non-uniformity is given for sources C3 and C4. The in-air measurements were performed with the applicator placed onto the film. Solid water was used as backscatter material for both in-air and in-phantom measurements. Radial source profiles of in-air and in-phantom measurements are shown in Figure 5-19. Both average radii and certificate dates of the ophthalmic sources are given in Table 5-9. Two of the older planar sources had a larger average radius (P5 and P4 with certificates from 1979 and 1985). For all data based on radiochromic film measurements, averaging over a $1 \mathrm{~mm}$ area around each pixel was applied to eliminate the effect of small hot spots or noise on evaluation of source non-uniformity.
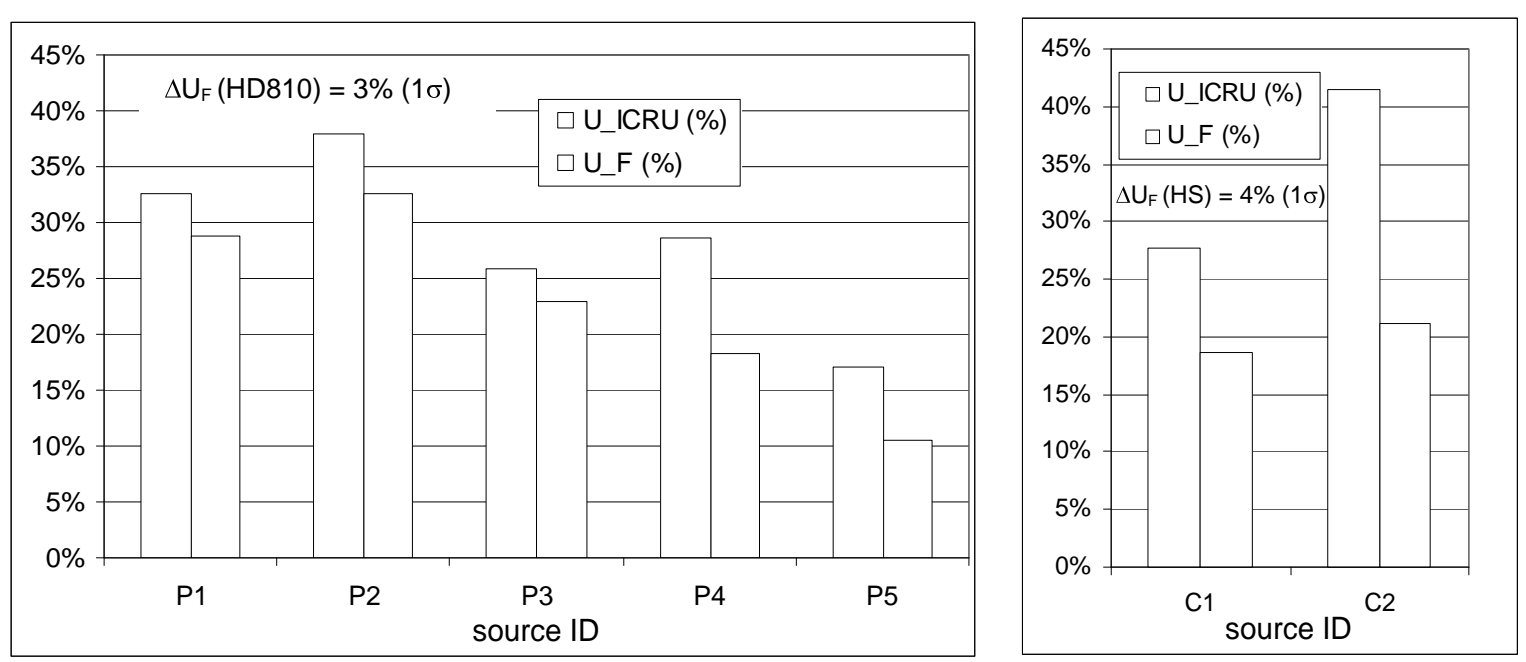

Figure 5-16 Source non-uniformity (\%) of the planar SIA.20 sources as measured with HD810 film at the source surface. Both $U_{\text {ICRU }}$ and $U_{F}$ are shown.

Figure 5-17 Source non-uniformity (\%) of the concave SIA. 6 sources as measured with HS at $z=3.4 \mathrm{~mm}$ in solid water.

Table 5-9 Average radius for the planar $(P)$ and concave $(C)$ Amersham sources.

\begin{tabular}{|c|c|c|c|c|c|}
\hline $\begin{array}{c}\text { Source } \\
\#\end{array}$ & $\begin{array}{c}\text { Certificate } \\
\text { date }\end{array}$ & $\mathbf{R}_{\mathbf{5 0}} \mathbf{( m m )}$ & $\begin{array}{c}\text { Source } \\
\#\end{array}$ & $\begin{array}{c}\text { Certificate } \\
\text { date }\end{array}$ & $\mathbf{R}_{\mathbf{5 0}}(\mathbf{m m})$ \\
\hline P1 & 2000 & 3.60 & C1 & 1993 & $5.40(\mathrm{SW})$ \\
\hline P2 & 2000 & 3.55 & C2 & 1992 & $5.02(\mathrm{SW})$ \\
\hline P3 & 1980 & 3.54 & C3 & 1992 & $6.91(\mathrm{~A})$ \\
\hline P4 & 1985 & 5.64 & C4 & 1983 & $5.95(\mathrm{~A})$ \\
\hline P5 & 1979 & 5.37 & \multicolumn{4}{|l}{}
\end{tabular}

$A=$ in air; $S W=$ in solid water; $C 1$ and $C 2$ are SIA. 6 sources.

Typical radial profiles of the strontium sources are given in Figure 5-18 (planar sources P1 and P5) and Figure 5-19 (concave source C2). The profiles and average radii of sources P1P3 were similar and differed strongly from sources P4 and P5 as demonstrated in Figure 5-18a and $b$ and by the data in Table 5-9. It is likely that Amersham may have changed the manufacturing procedure over the years, explaining the difference between both SIA.20 activity distributions. No solid water film measurements were performed on the concave 
sources with a deviating radius of curvature (C3 and C4), because no phantom with appropriate curvature was available. The film measurements on concave sources contain a convolution of geometry and non-uniformity. Therefore the numerical values of nonuniformity, as measured in a plane perpendicular to the z-axis, have a different meaning.
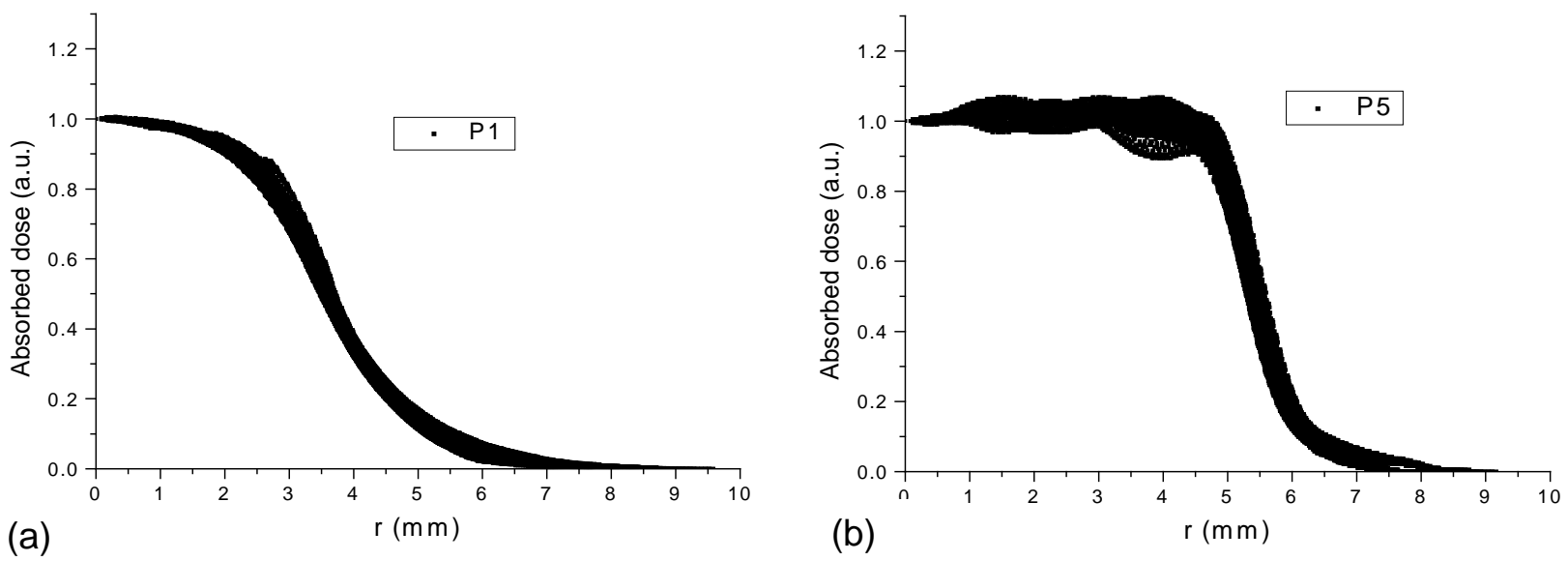

Figure 5-18 Radial dose distribution for SIA.20 sources P1 (a) and P5 (b) as measured with HD810 film at the surface.
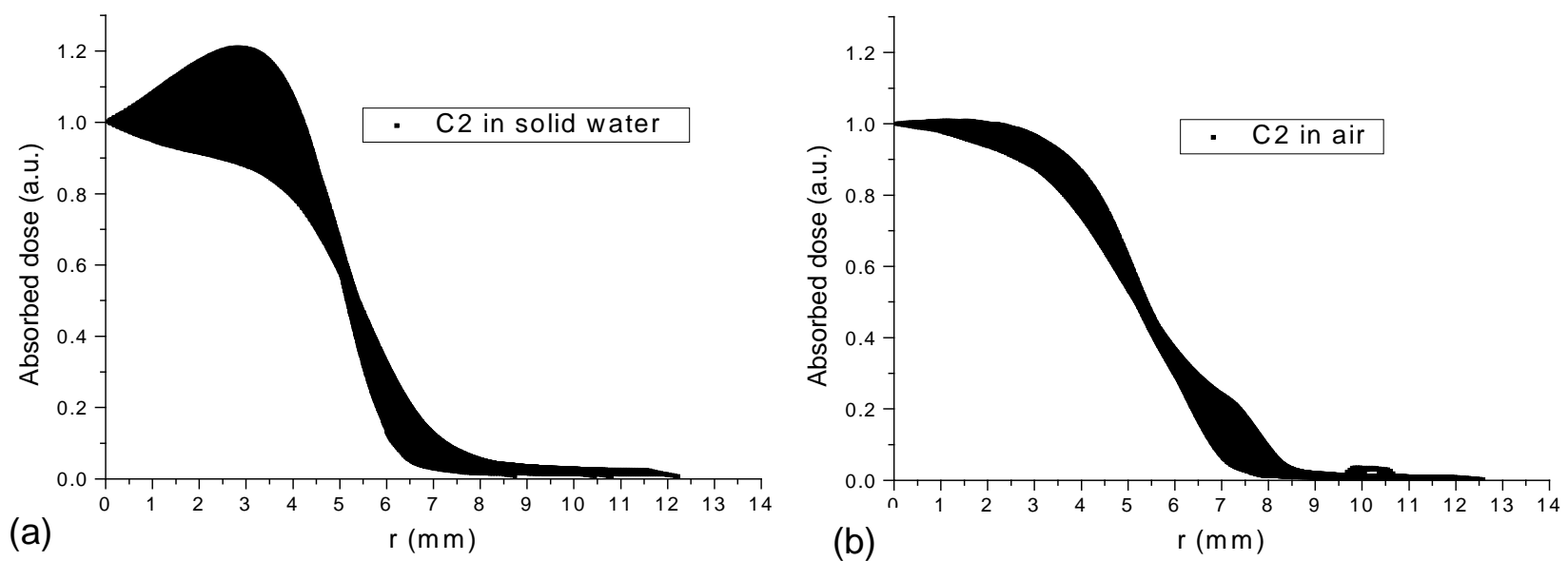

Figure 5-19 Radial dose distribution for SIA.6 source C2 in solid water (a) and in air (b) as measured with $\mathrm{HS}$ at $\mathrm{Z}=3.4 \mathrm{~mm}$.

Figure 5-19a shows large non-uniformity of source C2. Figure 5-19b shows the in-air measurement on this source. The curve is so strongly blurred that the source non-uniformity is not revealed. Emitted electrons have little interaction with air molecules before reaching the film surface. Because of this, a relatively large part of the source surface contributes to local film irradiation and most spatial information is lost. Therefore, the usefulness of in-air measurements on concave sources is questionable, despite the fact that these measurements are easy to perform when no phantom is available.

The profile of sources such as P1-P3 is symmetrical but not flat. Therefore non-uniformity is high; only one of the (planar) sources had a value for $U_{I C R U}$ below $20 \%$ as required by the ICRU. Graphs such as Figure 5-18 and Figure 5-19 are very suitable for evaluation of source (a)symmetry. In Section 3.2.2 the concept of source asymmetry was introduced in addition to source non-uniformity. Using this concept an asymmetrical activity distribution can be 
distinguished. The same area of the source is evaluated as in the ICRU concept $\left(0.8 \mathrm{R}_{50}\right)$. For instance source $\mathrm{C} 2$, which has a strong asymmetrical distribution, has a high asymmetry value. For all film measurements on strontium sources (except those in-air) the value of the source asymmetry is given in Figure 5-20. Source P3 is a typical example of a source with a very symmetrical activity distribution, but with high non-uniformity.

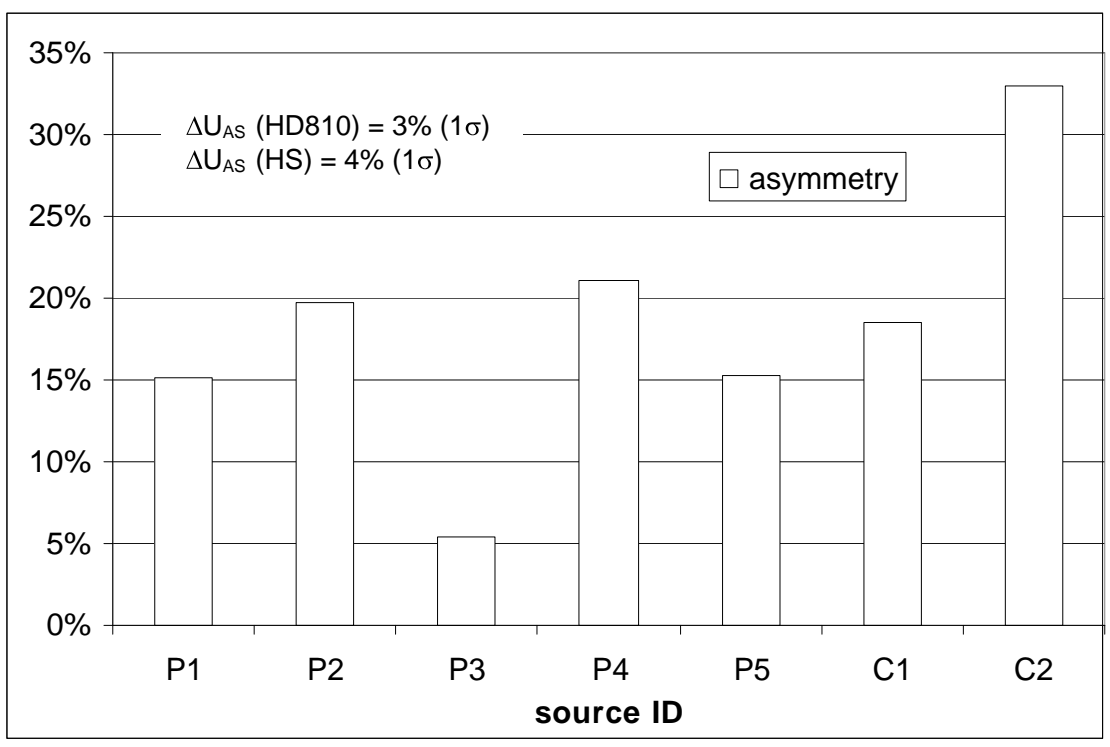

Figure 5-20 Source asymmetry $U_{A S}$ for ophthalmic strontium sources as measured with radiochromic film (HD810 for planar sources, HS for concave sources).

\subsubsection{Ophthalmic ruthenium sources}

The source strength of $10 \mathrm{BEBIG}{ }^{106} \mathrm{Ru} /{ }^{106} \mathrm{Rh}$ sources was measured at $z_{0}=2 \mathrm{~mm}$. Although only plastic scintillator measurements were intended for source strength measurements, diode measurements are also presented in the same figure (Figure 5-21), as these data were readily available. Averages and standard deviations of these measurements are given in Table 5-10. Both plastic scintillator and diode measurements in Figure 5-21 are normalized to $0 \%$.

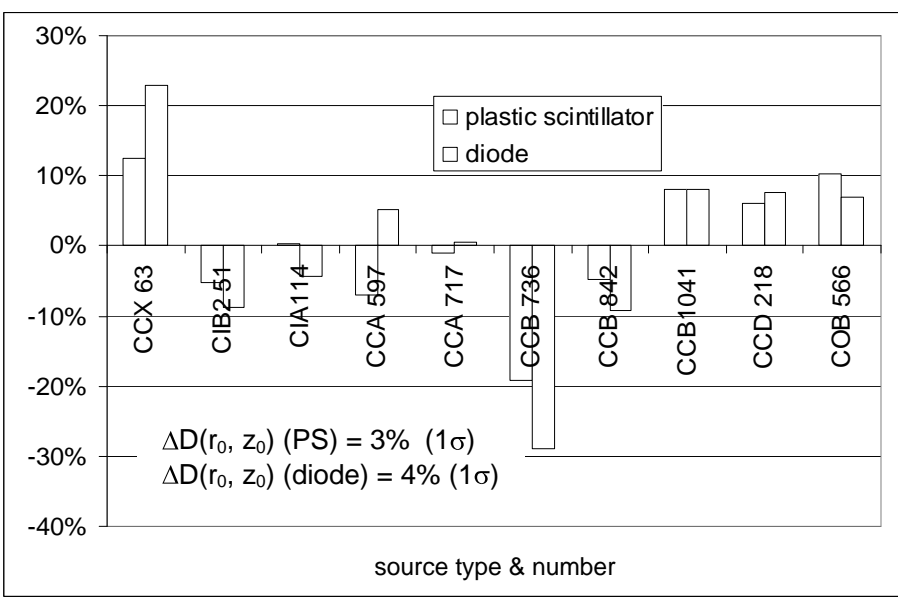

Figure 5-21 Relative difference (D-S2) in source strength between specification (S2) and detector $(D)$ for BEBIG ${ }^{106} \mathrm{Ru} /{ }^{106} \mathrm{Rh}$ sources as measured with plastic scintillator or diode at 2 $\mathrm{mm}$ in water (data normalized to $0 \%$ ).

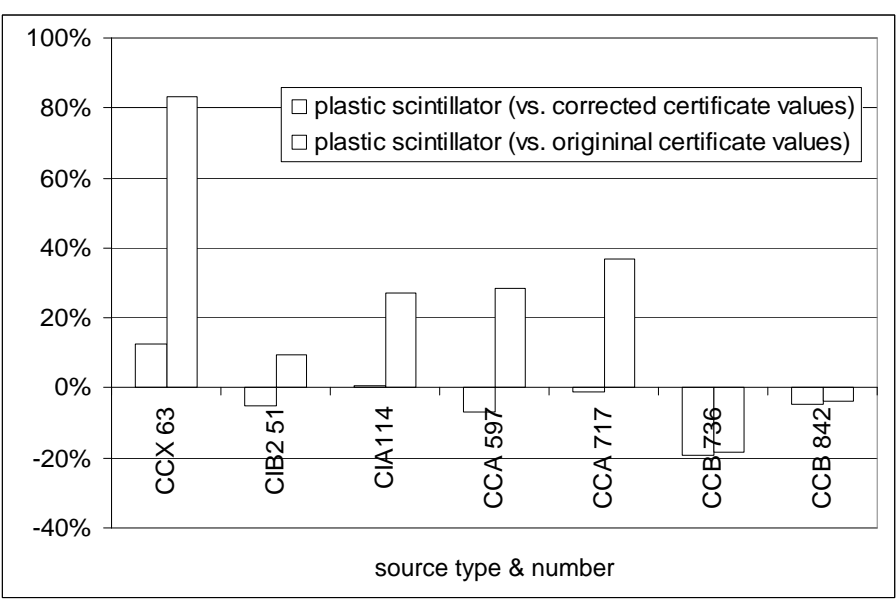

Figure 5-22 Relative difference (D-S1) or (D-S2) in source strength between original (S1) and corrected (S2) specification and detector (D) for BEBIG ${ }^{106} \mathrm{Ru}{ }^{106} \mathrm{Rh}$ sources as measured with a plastic scintillator at $2 \mathrm{~mm}$ in water. 
After BEBIG introduced a new (plastic scintillator) standard for its source calibrations in the spring of 2002, a correction table for the dose rate was distributed to all users for sources that were calibrated with the previous standard (letter dated 15-5-2002). These corrections were taken into account in Figure 5-21. Differences introduced with this correction are shown in Figure 5-22. For obvious reasons no normalization compared with the original certificate values was performed. Depending on the source type, the correction was smallest for the CCB (factor of 1.01 at $2 \mathrm{~mm}$, i.e. 1\% difference) and largest for the CCX (factor of 1.64 at 2 $\mathrm{mm}$ ). Some of the corrections at $5 \mathrm{~mm}$ were even larger than a factor of 2. CCB1041, CCD218 and COB566 were calibrated with the new BEBIG standard.

Table 5-10 Deviation and spread in measured source strength for the BEBIG sources. Distinction is made between sources calibrated with the old and new BEBIG standards.

\begin{tabular}{|c|c|c|}
\hline & Plastic scintillator & Diode \\
\hline $\begin{array}{c}\text { Sources calibrated with old BEBIG standard } \\
\text { (original certificate values) }\end{array}$ & $\begin{array}{c}23.3 \% \pm 32.9 \%(1 \sigma) \\
(\mathrm{n}=7)\end{array}$ & $\begin{array}{c}25.0 \% \pm 42.1 \%(1 \sigma) \\
(\mathrm{n}=7)\end{array}$ \\
\hline $\begin{array}{c}\text { Sources calibrated with old BEBIG standard } \\
\text { (corrected values) }\end{array}$ & $\begin{array}{c}-3.5 \% \pm 9.5 \%(1 \sigma) \\
(\mathrm{n}=7)\end{array}$ & $\begin{array}{c}-3.2 \% \pm 15.8 \%(1 \sigma) \\
(\mathrm{n}=7)\end{array}$ \\
\hline $\begin{array}{c}\text { Sources calibrated with new BEBIG standard } \\
\text { (n=3) }\end{array}$ & $\begin{array}{c}8.1 \% \pm 2.0 \%(1 \sigma) \\
(\mathrm{n}=3)\end{array}$ & $\begin{array}{c}7.5 \% \pm 0.6 \%(1 \sigma) \\
(\mathrm{n}=3)\end{array}$ \\
\hline
\end{tabular}

Figure 5-22 shows that the calibrations performed with the new standard were more consistent with the present measurements than those performed with the old standard (SD = $2 \%$ vs. SD $=10 \%$, even taking into account the correction table). An average difference of about $27 \%$ was introduced by the correction introduced by BEBIG, based on the data for the 7 old sources (compare "original" with "corrected" in Table 5-10.). This difference emphasizes the importance of independent verifications. The average of the corrected values $(-3 \%)$ was not consistent with the average of the new calibrations $(+8 \%)$ suggesting that the correction itself may not be correct, although only 3 sources with a new calibration were evaluated.

The difference between diode and the plastic scintillator measurements had a standard deviation of $4.2 \%$ (absolute value of differences taken into account). The maximum difference between diode and plastic scintillator measurements was $12.3 \%$. This value gives an indication of the relative consistency of diode and the plastic scintillator measurements. The difference can be explained by the positioning uncertainty introduced by measuring in water at $2 \mathrm{~mm}$. Because diode measurements were only performed by a single $60 \mathrm{~s}$ integration, there was no averaging through source repositioning. Uncertainty in source positioning may explain the largest part of the differences between both measurements. The change in diode sensitivity was not higher than $2 \%$, as far as could be determined with check source measurements. A change in diode sensitivity does therefore not explain such a large difference.

Non-uniformity of the ruthenium sources was determined with a diode at the source surface (and perpendicular to the surface) in water and with radiochromic film at $4.5 \mathrm{~mm}$ in solid water. Calculated non-uniformity $U_{F}$ at the surface is plotted in Figure 5-23 for both diode measurements and BEBIG calibration. BEBIG gives a number of measured dose rate values at the surface of the applicator. Although film measurements perpendicular to the source axis were performed, these are not included in the graph. Diode measurements were performed crosswise following $x$ - and $y$-axes, and from the certificate only the values of the corresponding diagonals were used for the calculation of non-uniformity. Measurements within a few $\mathrm{mm}$ of the cutouts were not included. The difference between the concepts of non-uniformity as introduced by the NCS and the ICRU is shown in Figure 5-24. The asymmetry of the sources was calculated from the certificate, diode and film measurements and is given in Figure 5-25. The same data set as for the non-uniformity was used. The film 
measurements that didn't suffer too much from artefacts or deviating source shapes were included in the graph, although the measurements were performed in a different plane than the diode measurements.

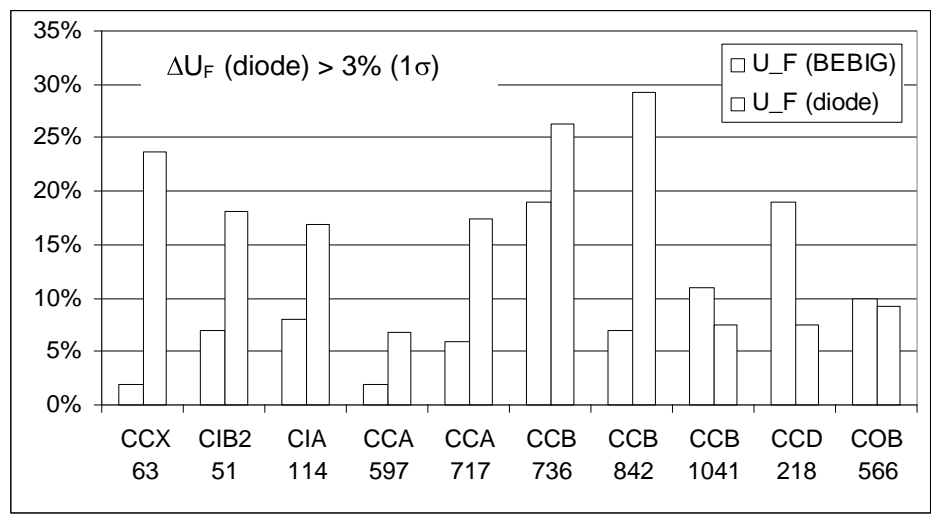

Figure 5-23 Non-uniformity $U_{F}$ of ruthenium sources as calculated from BEBIG's certificate surface dose rate values and from diode surface measurements in water.

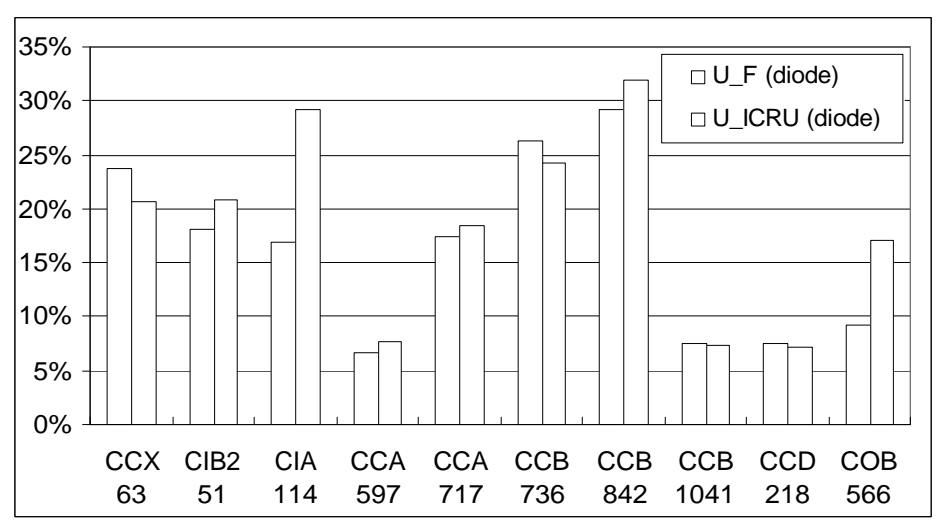

Figure 5-24 Comparison between $U_{F}$ and $U_{I C R U}$ for diode measurements on ruthenium sources.

For the calculation of the (BEBIG) values in Figure 5-23 the certificate was used. The differences between both measurements can be explained by the large measurement volume of the old BEBIG standard (2 $\mathrm{mm}$ diameter and depth), in which hot spots are averaged out. In contrast to the present measurements, the BEBIG measurements were performed with the detector parallel to the z-axis. The present measurements on the recently calibrated sources are more consistent with the certificate, indicating that the new BEBIG standard is more accurate than the old standard. Some of the values given by the recent BEBIG calibration result in an even higher non-uniformity, because more measurement points are given on the BEBIG certificate than in the present measurements.

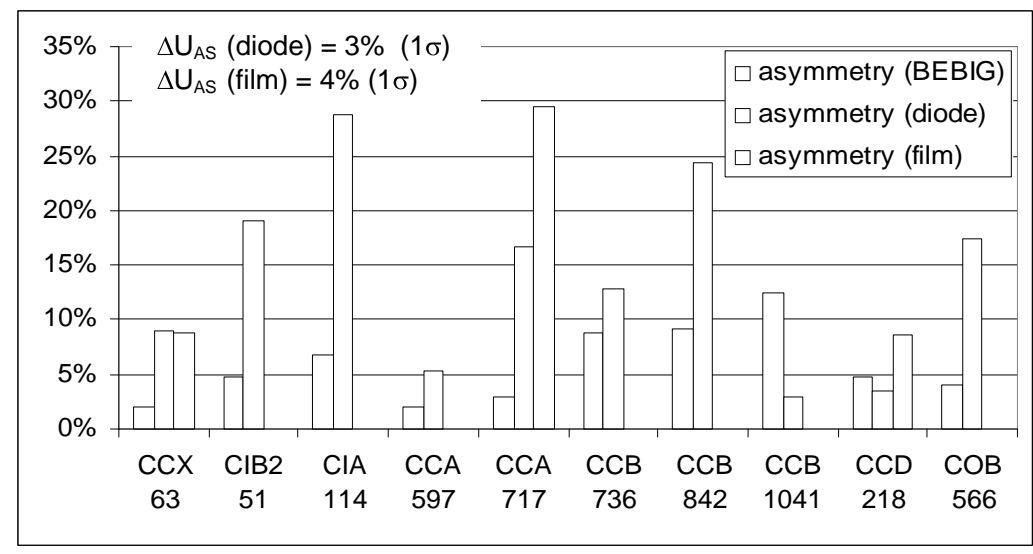

Figure 5-25 Source asymmetry $U_{A S}$ of ruthenium sources as calculated from certificate (BEBIG), film and diode measurements.

The (asymmetry) results presented in Figure 5-25 show similar differences between the present measurements and the certificate values as seen in Figure 5-23. Where possible, film data are also included. As an example, in Figure 5-26 a radial profile of a COB source is given. Film measurements on such sources have several pitfalls. Firstly, film measurements on large sources give very large non-uniformities due to the varying film-source surface distance (between 0 and $4.5 \mathrm{~mm}$ ). Secondly, for film measurements source positioning is critical because a source that is not fully aligned parallel to the film will result in a non- 
symmetrical source profile. One can imagine that profiles of sources with a cutout like the $\mathrm{COB}$ are not at all useful for quantitative evaluation of source non-uniformity or asymmetry (see Figure 5-26). Finally, when making film measurements on ruthenium sources, the eyes of the source limit the position where the film can be placed. The inset of Figure 5-26 shows that the edge of the film is also visible on the film scan. Such edges may cause artefacts when a quantitative evaluation of the scan is attempted.

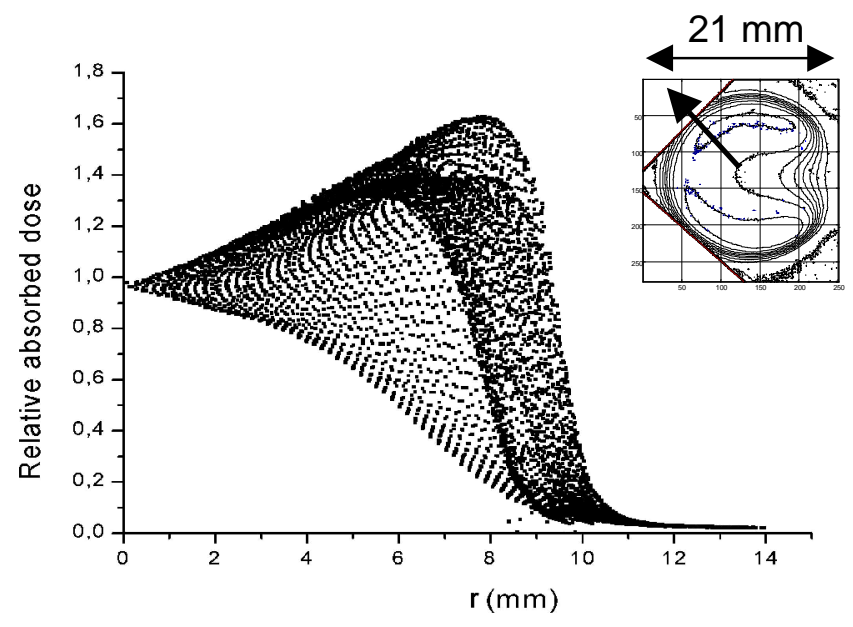

Figure 5-26 Radial dose distribution for the COB566. Because of the cutout in the applicator the center of the absorbed dose is not in the middle. The radial distribution is not symmetrical due to the cutout in the source. Inset: isodoses for same applicator. 


\subsection{Evaluation of tested detectors}

This section provides an evaluation of the detectors that were tested during the on-site measurements. Their suitability for strength or uniformity measurements is discussed. The recommendations given in Section 6.3 are mainly based on this section.

\section{Well-type ionization chamber}

Because the well-type ionization chamber has the highest reproducibility of all detectors in beta dosimetry, it is very suitable for strength verifications. Although a chamber insert is required, the fact that no phantom is needed for measurements is an advantage. A disadvantage is that these measurements need to be performed in combination with for example film measurements to determine source uniformity. Due to the required amount of signal the chamber insert must be kept thin for beta emitters. The insert of the IVB1000 is not part of the chamber and is therefore more vulnerable. Currently, clinical catheters are used for measurements on the Novoste sources with the IVB1000 chamber. These catheters are made for single use and therefore are vulnerable, especially the $3.5 \mathrm{~F}$ catheter. A dedicated rigid physics catheter would be more suitable for these measurements.

One should be aware of the disadvantage of using a pressurized ionization chamber. Although these chambers sometimes are available in a nuclear medicine department, their stability is not guaranteed due to possible gas leakage. The high response that is needed for nuclear medicine applications is not important, because brachytherapy sources have much higher source strength and vented ionization chambers give sufficient signal. For these reasons the IAEA recommends using a vented chamber [3]. Application of a (T,p)-correction is necessary.

Plastic scintillator

Advantages of the plastic scintillator are that it can be used for high and low dose rate sources and for strength, depth dose and uniformity measurements. It also has a high degree of water equivalence. It is expected that the detector can be used for uniformity measurements on ruthenium sources as well, although no experiments for this purpose were performed.

Few institutes have a plastic scintillator, although it is readily available. Other disadvantages are mainly related to the detector stability. Measurements showed that the use of a check source (as PTW recommends) is essential for use of the detector. Two types of variations in detector sensitivity were found: short-term and long-term changes. The slow and long-term decrease of detector sensitivity can be corrected for by check source measurements. Shortterm changes also occur, possibly caused by environmental changes or changes in probe position. Short-term changes up to $21.6 \%$ were observed, although most were below $3 \%$ within the 40 minutes time frame. A difference below 2 or $3 \%$ is recommended. For more control of short-term changes in detector sensitivity it is also possible to reduce the time between two check source measurements.

Analysis of the plastic scintillator measurements on the Guidant sources showed a large uncertainty in the positioning of the phosphorus source in the PTW phantom. Even with small changes in the shape of the physics coupler, rather significant changes in detector reading were apparent (up to $6 \%$ ). This can be correlated to the diameter of the hole in the phantom as designed by PTW. At the time of writing PTW has acknowledged this problem and the diameter of the hole has been reduced from $0.60 \pm 0.02 \mathrm{~mm}$ to $0.53 \pm 0.02 \mathrm{~mm}$.

\section{Plane-parallel ionization chamber}

Because the plane-parallel ionization chamber has low spatial resolution and limited sensitivity, it is not very suitable for calibrating most beta sources. The detector can be used for relative source strength checks on high dose rate ophthalmic strontium sources, as long as the measurement set-up is reproducible. If different planar sources need to be compared, 
differences in source geometry and activity distribution will strongly affect detector response due to the large electrode diameter. The detector is available in most radiotherapy departments.

\section{Diode}

The low degree of water equivalence can be regarded as a disadvantage of the diode. The detector is, however, part of the standard equipment of most radiotherapy departments and has relatively high spatial resolution. No warm-up time is needed and the detector can also be used in water. Although the diode was not primarily selected for source strength measurements, results for ruthenium sources were similar to those obtained with plastic scintillators. The accuracy of positioning the diode can be improved by introducing a solid phantom for measurements on both clinical and check sources. Averaging over multiple integrating measurements will also contribute to a more accurate result. Under these conditions use of a diode for measurements on ophthalmic strontium sources and intravascular sources may be considered, but further studies are required.

\section{Radiochromic film}

The most attractive property of radiochromic film is its high spatial resolution. The need for film densitometry is a disadvantage that all users are aware of, although non-uniformities larger than $10 \%$ can be observed with the naked eye. Variations in thickness of the sensitive layer have been reported for MD55-2. This effect was also noticed for HS film, where it caused artificially high source non-uniformity. For HD810 this effect was not noticed, nor found in the literature. The effects of variations in thickness of the sensitive layer are minimized when the film is cut in the direction in which the coating is applied [42]. This effect can be corrected for by the application of the double exposure technique [42]. If HS is used, detachment of the layers may be expected near the edge of the film (up to $1 \mathrm{~mm}$ ), because of the cutting. This phenomenon can be explained from the composition of the film, in which an adhesive layer, as used for MD55-2, is omitted. In spite of these disadvantages, HS is attractive as a detector for low dose rate sources because of its high sensitivity. The disadvantages mentioned and the differences in film sensitivity should determine the choice for a specific type of film.

For quantitative evaluation of the uniformity of intravascular and planar ophthalmic sources radiochromic film can be used. In the latter case the user should be aware that the nonuniformity $U_{\text {ICRU }}$ may exceed the maximum $20 \%$ demanded by the ICRU and the IAEA [2,3]. Radiochromic film should not be applied for quantitative evaluation of non-uniformity of concave sources. For estimating the asymmetry of ophthalmic sources radiochromic film is a suitable detector.

Although some institutes have experience with routine source calibrations using HD810 film, within the scope of the present study, use of radiochromic film for absolute measurements was not investigated. Therefore, presently, using film for this purpose cannot be recommended. Those who still like to use radiochromic film for absolute measurments should be aware that additional film calibrations are necessary and that these measurements are more time consuming than those with for example a well-type ionization chamber or plastic scintillator. A film phantom should be manufactured with small tolerances, because of the steep dose gradient of the sources. Using two parallel plates without a channel for line source calibrations will not give appropriate results. 


\subsection{Application of action levels on investigated sources}

In the next chapter (Section 6.4) action levels are formulated for maximum deviations in source strength, for maximum non-uniformity and asymmetry. If these action levels are applied to the source strength of the clinical sources that were investigated, several sources do not satisfy the requirement. The numbers are given in Table 5-11. Differences can be attributed to:

- uncertainty in the measurement

(size of the phantom channel for the plastic scintillator measurements on the ${ }^{32} \mathrm{P}$ source)

- absence of a source certificate (planar ${ }^{90} \mathrm{Sr} /{ }^{90} \mathrm{Y}$ source)

- deviating source geometry (planar ${ }^{90} \mathrm{Sr} /{ }^{90} \mathrm{Y}$ source)

- large uncertainties with old manufacturer's standard (concave ${ }^{106} \mathrm{Ru} /{ }^{106} \mathrm{Rh}$ source)

For all currently available clinical sources and available detectors, it is possible to deal with these problems.

Table 5-11 Number of sources with a strength deviating more than $10 \%$ from the mean value (normalized data used as presented in Section 5.4).

\begin{tabular}{|c|c|c|c|}
\hline Technique & $\begin{array}{c}\text { Well-type ionization } \\
\text { chamber }\end{array}$ & Plastic scintillator & $\begin{array}{c}\text { Plane-parallel } \\
\text { ionization chamber }\end{array}$ \\
\hline IVBT & $0 / 24$ & $1 / 24^{*}$ & n.a. \\
\hline Ophthalmic $\mathrm{Sr}$ & n.a. & $2 / 9^{\dagger}$ & $2 / 5^{\dagger}$ \\
\hline Ophthalmic $\mathrm{Ru}$ & n.a. & $2 / 10^{\ddagger}$ & n.a. \\
\hline
\end{tabular}

The number of sources with a non-uniformity or asymmetry higher than the action level is summarized in Table 5-12. The action levels specified in Section 6.4 are used: $U_{F}<10 \%$ for line sources, $U_{F}<30 \%$ and $U_{A S}<20 \%$ for ophthalmic sources. As can be seen in the table, some sources display significant non-uniformity or asymmetry. The number of sources that exceed the action levels as well as the deviations regarded clinically relevant are taken into consideration in the choice of the action levels.

Table 5-12 Number of sources with a non-uniformity or asymmetry larger than the action level.

\begin{tabular}{|c|c|c|c|}
\hline Technique & $\mathbf{U}_{\mathrm{F}}>\mathbf{1 0} \%$ & $\mathbf{U}_{\mathrm{F}}>\mathbf{3 0} \%$ & $\mathrm{U}_{\mathrm{AS}}>\mathbf{2 0} \%$ \\
\hline IVBT & $2 / 24$ & $1 / 5$ & $1 / 5$ \\
\hline $\begin{array}{c}\text { Ophthalmic } \mathrm{Sr} \\
\text { (planar) }\end{array}$ & & & $1 / 2$ \\
\hline $\begin{array}{c}\text { Ophthalmic } \mathrm{Sr} \\
\text { (concave) }\end{array}$ & & $0 / 10$ & $2 / 10$ \\
\hline Ophthalmic Ru & & & \\
\hline
\end{tabular}

\footnotetext{
"Due to source channel problem.

${ }^{\dagger}$ Due to difference in source size and absence of certificate.

${ }^{\ddagger}$ Both with old BEBIG standard.
} 


\section{NCS recommendations for quality control of beta sources}

This chapter contains an overview of procedures for a quality control programme of beta sources. In Section 6.1 the scope of dosimetry of beta sources is given. Measurement concepts and corresponding reference points for source strength determinations are introduced in Section 6.2, and recommendations on suitable detectors are provided in Section 6.3. In Section 6.4 dosimetric action levels are set. Sections 6.5 and 6.6 present other physical properties and safety procedures that should be part of a quality control programme, including recommendations on the frequency of such tests.

\subsection{Scope of dosimetry of beta sources}

The NCS recommends determining the following dosimetric parameters:

Minimum requirements:

- absolute source strength ${ }^{*}$

- active source length (line sources) or average radius (ophthalmic sources)

- source non-uniformity

- source asymmetry (ophthalmic sources)

Suggested additional measurements:

- depth dose in the case of a known change in source design

The parameters are specified in Section 6.2.

\subsection{Recommended reference points and source parameters}

The $r$ and $z$ axis and the reference points $r_{0}$ and $z_{0}$ in this section are defined in the coordinate system as depicted in the figures of Section 3.1. A more extensive description of the parameters of interest is given in Section 3.2.

\subsubsection{Intravascular sources}

\section{Active Source Length (ASL)}

The active source length is the distance between the $50 \%$ points of the longitudinal dose distribution at $r_{0}=2 \mathrm{~mm}$ (see Figure 3-2). The $100 \%$ level is set by the maximum dose.

\section{Source strength}

The source strength $\dot{D}\left(r_{0}\right)$ of intravascular sources is defined as the average of the absorbed dose rate to water at $r_{0}=2 \mathrm{~mm}$ along the source axis. The average should be determined in the ASL, excluding a margin from the $50 \%$ points of $M_{P}=2.5 \mathrm{~mm}$ for ${ }^{32} \mathrm{P}$ sources and $M_{S r}=3.0 \mathrm{~mm}$ for ${ }^{90} \mathrm{Sr} /{ }^{90} \mathrm{Y}$ sources. $\dot{D}\left(r_{0}\right)$ can, in practice, be determined by:

a) a well-type ionization chamber measurement, or

b) a series of measurements in a representative number of points along the source axis.

Source non-uniformity

The source non-uniformity of intravascular sources is expressed as

$$
U_{F}=\max \left(\left|\dot{D}_{\min }-\dot{D}_{\text {avg }}\right|,\left|\dot{D}_{\max }-\dot{D}_{\text {avg }}\right|\right) / \dot{D}_{\text {avg }} \times 100 \%
$$

\footnotetext{
* Using a detector calibrated at the national standards institute. If no such absolute calibration can be obtained, the consistency of detector readings and certificates of two or more different sources should be checked (see Chapter 5).
} 
in which:

$$
\begin{aligned}
& U_{F}=\text { source non-uniformity (\%) } \\
& \dot{D}_{\max }=\text { maximum (relative) absorbed dose rate within ASL-2M } \\
& \dot{D}_{\min }=\text { minimum absorbed dose rate within ASL-2M } \\
& \dot{D}_{\text {avg }}=\text { average absorbed dose rate within ASL-2M }
\end{aligned}
$$

The NCS recommends evaluating the source non-uniformity at $r_{0}=2 \mathrm{~mm}$ over the active source length minus a margin $M_{P}=2.5 \mathrm{~mm}$ for ${ }^{32} \mathrm{P}$ sources, or $M_{S r}=3.0 \mathrm{~mm}$ for ${ }^{90} \mathrm{Sr} /{ }^{90} \mathrm{Y}$

sources. The average absorbed dose rate $\dot{D}_{\text {avg }}$ is equal to the source strength $\dot{D}\left(r_{0}\right)$ if it is determined with a calibrated detector.

\subsubsection{Ophthalmic sources}

\section{Source strength}

The source strength $\dot{D}\left(r_{0}, \mathrm{z}_{0}\right)$ of all planar and concave sources should be expressed as an absorbed dose rate in water at $\left(r_{0}, z_{0}\right)=(0,2) \mathrm{mm}$.

\section{Average radius $(R 50)$}

The average radius $\left(R_{50}\right)$ is defined as the mean radius of the $50 \%$ isodose contour of a dose distribution in which $100 \%$ is given by the maximum absorbed dose.

\section{Source non-uniformity}

The source non-uniformity (flatness) is expressed by:

$$
U_{F}=\max \left(\left|\dot{D}_{\min }-\dot{D}_{r=0}\right|,\left|\dot{D}_{\max }-\dot{D}_{r=0}\right|\right) / \dot{D}_{r=0} \times 100 \%
$$

In this formula $\dot{D}_{r=0}$ is the absorbed dose rate as determined on the z-axis $(r=0) . \dot{D}_{\text {min }}$ and $\dot{D}_{\text {max }}$ are determined in an area with a maximum radius of $0.8 \mathrm{R}_{50}$.

\section{Source asymmetry}

For evaluation of the asymmetry of ophthalmic sources the quantity $U_{A S}$ is used:

$$
U_{A S}=\max \left(\left\{\dot{D}_{\max }(r)-\dot{D}_{\min }(r)\right\} / \dot{D}_{\text {avg }}(r)\right) \times 100 \%
$$

To assess $U_{A S}$, the variation of the dose is determined for all circles with radii $r$, up to $r=0.8 R_{50}$. The maximum observed variation determines the value of the asymmetry $U_{A S}$. The source asymmetry and non-uniformity can usually be determined using the same data set.

\section{Choice of measurement position}

The average radius, source non-uniformity and asymmetry are defined at a constant distance of the source surface. It is recommended that these parameters are determined as close to the surface as possible. For concave sources such a measurement position may be problematic because in water measurements are not always acceptable. Data from (film) measurements performed in a plane perpendicular to the source axis $z$ can be used to estimate the source asymmetry and non-uniformity. In those measurements, the asymmetry of a source may be underestimated because blurring can occur due to the large distance between the source surface and film. Action levels formulated for the source asymmetry 
according to the definition in this section are expected to hold also for measurements in a plane. On the other hand, the source non-uniformity may well be overestimated due to the variable distance between source surface and film. A perfectly uniform concave source will give a non-uniformity larger than zero in a plane. For this reason, no action levels are formulated for the source non-uniformity determined in a plane. Our experience shows that the non-uniformity calculated from the film measurements on two concave strontium applicators satisfied the action levels presented in Section 6.4. This experience is, however, too limited to support a recommendation.

\subsection{NCS recommendations for suitable measurement systems}

Based on the evaluation in Section 5.5, the NCS has selected a number of techniques that can be used for (1) absolute calibrations and/or (2) determination of the uniformity of beta sources. A summary of the recommendations is given in Table 6-1. The terms "suitable detectors" and "other possible detectors" are used to make a distinction between detectors that are regarded best for that application and other suitable, but less optimal detectors. The recommended measurement procedures are given in Appendix 10. Details on possible phantoms are provided in Section 5.1.3. Drawings of the phantoms are given in the appendices.

The recommendations in this section are meant to indicate those phantoms and detectors that are regarded to be suitable for source strength or uniformity measurements. The recommendations are limited by the set of detectors that were available for the survey. Detectors that were not investigated may also be suitable as long as they satisfy the same criteria.

\subsubsection{Detectors suitable for intravascular brachytherapy sources}

The NCS recommends performing source strength measurements on intravascular brachytherapy sources with a plastic scintillator or a (vented) well-type ionization chamber. For uniformity measurements on intravascular sources the use of GafChromic film is recommended. HD810 film is the best choice for evaluation of source uniformity. However, HD810 is not very practical for measurements on ${ }^{32} \mathrm{P}$ sources because of the long irradiation times that need to be introduced manually into the afterloader program. MD55-2 and HS films can also be used, but may show variations in the thickness of the sensitive layer. A plastic scintillator can be used for uniformity measurements, but the spatial resolution of this detector is limited. For measurements with the Optidos phantom on Novoste sources, only measurements with a spacing of $10 \mathrm{~mm}$ are possible.

\subsubsection{Detectors suitable for ophthalmic strontium sources}

Ophthalmic strontium sources usually are replaced with an interval of 10 years or more. Therefore the NCS recommends sending these sources to the national standards institute for calibration before clinical application. Institutes equipped with a calibrated plastic scintillator can do their own measurements. Every 3 years it should be verified that the source strength decreases in accordance with the natural decay. For this purpose a plane-parallel ionization chamber or a plastic scintillator can be used.

The NCS recommends checking the uniformity of strontium sources with radiochromic film. GafChromic HD810 is the most suitable film for planar strontium sources (for example a dose rate of $0.1 \mathrm{~Gy} \mathrm{~s}^{-1}$ requires half an hour irradiation time for an absorbed dose of $200 \mathrm{~Gy}$ ). For measurements on sources with a lower dose rate, such as the concave strontium sources, MD55-2 or HS can be used. Non-uniformity and asymmetry of the sources can be determined quantitatively by densitometry [33]. Diodes and plastic scintillators are not suitable for uniformity measurements, because it is not desirable to perform these measurements in water. In addition, the size of the active area is small compared with 
ruthenium sources.

\subsubsection{Detectors suitable for ophthalmic ruthenium sources}

For strength measurements on ruthenium sources use of a plastic scintillator is advised. Diode measurements in water are not recommended for this purpose, since they did not offer a sufficiently low uncertainty (see discussion in Chapter 5).

Non-uniformity and asymmetry of ruthenium sources can be determined best by diode or plastic scintillator measurements in water at the source surface. Radiochromic film can be used for verification of the symmetry of the activity distribution in a plane perpendicular to the source axis. Quantitative evaluation of these data is less straightforward than the suggested diode or plastic scintillator measurements, because the scans require further analysis.

GafChromic MD55-2 and HS are the most suitable films for this application, because they have the highest sensitivity. In the case of depth dose measurements, the cutting artefacts are much smaller for MD55-2 than for HS.

Table 6-1. Suitable detectors for determination of strength, uniformity and depth dose of sealed beta sources.

\begin{tabular}{|c|c|c|c|c|c|c|c|c|c|}
\hline & & $\begin{array}{c}\text { Plastic } \\
\text { scintillator }\end{array}$ & $\begin{array}{l}\text { Well-type } \\
\text { ionization } \\
\text { chamber }\end{array}$ & $\begin{array}{l}\text { HD810 } \\
\text { film }\end{array}$ & $\begin{array}{l}\text { MD55-2 } \\
\text { film }\end{array}$ & $\begin{array}{l}\text { HS } \\
\text { film }\end{array}$ & Diode & $\begin{array}{c}\text { Plane- } \\
\text { parallel } \\
\text { ionization } \\
\text { chamber }\end{array}$ & $\begin{array}{c}\text { Extrapolation } \\
\text { chamber at } \\
\text { standards } \\
\text { institute }\end{array}$ \\
\hline \multirow{3}{*}{$\begin{array}{c}\text { Intravasc. } \\
\text { BT }\end{array}$} & $S S$ & $\bar{X}$ & $\bar{x}$ & & & & & & \\
\hline & $U_{F}$ & $X$ & & $X$ & $x$ & $x$ & & & \\
\hline & $P D D$ & $X$ & & $X$ & $x$ & $x$ & & & \\
\hline \multirow{4}{*}{$\begin{array}{c}\text { Ophthalmic } \\
\mathrm{Sr}\end{array}$} & $S S$ & $\bar{X}$ & & & & & & $X^{*}$ & $X$ \\
\hline & $U_{F}$ & & & $X$ & $x$ & $x$ & & & \\
\hline & $U_{A S}$ & & & $X$ & $x$ & $x$ & & & \\
\hline & $P D D$ & $X$ & & $X$ & $X$ & $x$ & & & \\
\hline \multirow{4}{*}{$\begin{array}{c}\text { Ophthalmic } \\
\text { Ru }\end{array}$} & $S S$ & $\bar{X}$ & & & & & & & \\
\hline & $U_{F}$ & $X$ & & & & & $X$ & & \\
\hline & $U_{A S}$ & $X$ & & $x$ & $x$ & $x$ & $X$ & & \\
\hline & $P D D$ & $X$ & & $x$ & $\mathrm{X}$ & $x$ & $X$ & & \\
\hline
\end{tabular}

$S S=$ Source strength measurement; $U_{F}=$ uniformity (flatness); $U_{A S}=$ asymmetry; $P D D=$ Percentage Depth Dose; $X$ = suitable detector; $x=$ other possible detector

\subsubsection{Detector calibration}

Detectors (well-type ionization chamber, plastic scintillator) used for absolute dosimetry should be calibrated in absorbed dose to water at a standards laboratory. Separate calibration factors should be obtained for each type of source.

The calibration factor of a plastic scintillator needs to be verified at regular times due to possible sensitivity variations in time. This can easily be performed by measurements with a check source. The calibration of an ionization chamber needs to include a correction for temperature and pressure.

For checks of relative source strength, a detector (e.g. plane-parallel ionization chamber) should be used in a reproducible configuration such as described in Appendix 8, but no separate calibration is required. The detector reading can then be compared with an earlier detector reading combined with a source decay correction.

\footnotetext{
Relative source strength measurements on one source (type), planar sources only.
} 


\subsubsection{Importance of phantom tolerances}

Part of the total measurement system is the phantom that is used. Tolerances well below 0.1 $\mathrm{mm}$ should be used in manufacturing these phantoms. The dimensions of a phantom should be verified to gain insight in the measurement uncertainties that are introduced by this part of the total measurement system.

\subsubsection{Recommendations for film densitometry}

When radiochromic film is used for relative measurements, each batch should be calibrated in-house to establish the relation between optical density (as determined through the densitometer) and the absorbed dose. Each batch of film and every scanner should be considered independently. The use of a transmission scanner with a color depth of more than 8 bits/color is recommended, because the 256 levels of an 3x8 bits RGB (Red-GreenBlue) color coded file give (discrete) steps in the order of $2 \%$ due to the logarithm that is used for the calculation of the absorbed dose.

\subsubsection{Measurement uncertainties}

Given the recommended detectors, setup and procedures we estimate that a combined standard uncertainty of $4 \%(1 \sigma)$ can at least be achieved for the (relative) source strength, non-uniformity and asymmetry. The total measurement system should be tested in terms of e.g. reproducibility and mechanical tolerances to assure that the total combined standard uncertainty of the system is below this value of $4 \%$. Under these conditions action levels can be set and maintained as described in the following section.

\subsection{NCS recommended action levels}

In this section recommendations are given for the maximum deviations in source strength, maximum non-uniformity and maximum asymmetry.

\subsubsection{Overview of literature}

Few published recommendations are available regarding the maximum difference between specified and measured source strength for beta sources. The ICRU report on beta rays and low-energy photons [2] is the only report in which a maximum deviation is mentioned explicitly, though carefully worded. The action level is $\pm 15 \%$. The EVA GEC ESTRO group [8] gives a suggested tolerance level in source strength reproducibility of $\pm 5 \%$ for beta line sources. The corresponding action is not specified.

The only recommendation regarding deviations in active source length is given by the EVA GEC ESTRO group. A maximum deviation in of $\pm 1 \mathrm{~mm}$ is recommended.

For verification of source uniformity more recommendations are available. Most recommendations originate from the AAPM TG 60 report [4] that covers line sources for intravascular brachytherapy. In this report a uniformity better than $\pm 10 \%$ is recommended over two-thirds of the source length. The DGMP [5] subscribes this recommendation in its report 16. The EVA GEC ESTRO suggests to use a wider margin of $\pm 20 \%$ to include measurement uncertainties. The ICRU [2] and IAEA [3] have adapted the AAPM recommendations for line sources. They use a somewhat different concept that expresses the difference between maximum and minimum dose, as we described earlier in Section 3.2. For both line and ophthalmic sources the ICRU and IAEA recommend a maximum source non-uniformity $U_{I C R U}=20 \%$.

\subsubsection{NCS recommendations for action levels}

This section provides recommendations on maximum deviations in source strength as well 
as recommendations on maximum non-uniformity and asymmetry for both intravascular and ophthalmic sources. A summary of the NCS recommendations, concerning action levels, is given in Table 6-2. The underlying concepts are summarized in Section 6.2.

Several factors were taken into account in the choice of action levels.

1. the width of the therapeutic window (see Section 3.5)

2. uncertainties in detectors and measurement procedures

3. manufacturing tolerances that are considered to be achievable

If an action level is exceeded, the measurement should be repeated, in order to minimize the possibility that either detector or setup are responsible for the deviation rather than the calibration of the manufacturer. The NCS recommends that sources with a non-uniformity or asymmetry above the action level are not used for clinical application. The recommended actions in the case of source strength deviations are specified in the following sections and differ per source type.

\section{Action levels applied on source strength}

The action level for source strength presented in this chapter can be used twofold. Firstly, if a detector is calibrated at a primary standards institute, the action level can be directly applied. Both detector and source calibrations need to have the same traceability. The action level does not take into account differences between primary standards and does also not take into account the uncertainty in absolute absorbed dose such as the $\pm 15 \%(2 \sigma)$ specified by NIST.

The second situation exists if no absolute detector calibration is available. Then a relative source strength comparison needs to be done. The reference level used for comparison can be the measured source strength of previous sources (averaged). A strength comparison with the source of another institute can be done if no previous sources are available. In the survey presented in Chapter 5 the largest differences were found for the source strength of

the Guidant sources and the BEBIG sources. For example, comparison of $\dot{D}\left(r_{0}, z_{0}\right)$ for Guidant source $4(+6 \%)$ and source $3(-9 \%)$ would lead to rejection of source 3 , if source 4 was the first source measured. If the $0 \%$ level would be the reference level, then source 3 would be accepted. The spread in the relative source strength difference for the Guidant and BEBIG sources should be lower for the current sources, due to a better phantom (Guidant) and a new standard (BEBIG). A $\pm 10 \%$ tolerance should be able to cope with this spread. Therefore, the NCS recommends to apply the action level of $\pm 10 \%$ for source strength on both absolute measurements with calibrated detectors and on relative strength comparisons.

\section{Action levels for intravascular brachytherapy sources}

The source strength $\dot{D}\left(r_{0}\right)$ of intravascular brachytherapy sources should be within $\pm 10 \%$ of the value specified by the manufacturer, corrected for source decay. In the case of higher and reproducible deviations, the manufacturer should be contacted in order to resolve the cause of the discrepancy. It is advisable to ask the manufacturer for a review of the source calibration. If deviations larger than $5 \%$ are found with a well-type ionization chamber, the user should be alerted that something in the calibration chain or in his own measurements may have gone wrong.

The non-uniformity $U_{F}$ should be less than $10 \%$. A maximum deviation in active source length of $1 \mathrm{~mm}$ is tolerated, also for the total active source length of stepping sources. If during a visual check of deviations in step size are observed larger than $1 \mathrm{~mm}$, the source should also be sent back (this is in accordance with Guidant's QC guidelines).

Regarding the percentage depth dose no demands are made. Under normal circumstances no changes need to be expected in these properties. However, it is in the interest of the 
customer to know and, if possible, to verify the depth dose characteristics of a source in the case of changing circumstances. An example may be a change in the source design or the manufacturing process.

\section{Action levels for ophthalmic strontium sources}

Users of ophthalmic strontium applicators are recommended to have their source independently calibrated at a standards laboratory. If a calibrated plastic scintillator is available, it can be used as well. The measured source strength $\dot{D}\left(r_{0}, z_{0}\right)$ should be within $\pm 10 \%$ of the value specified by the manufacturer, corrected for source decay. In the case of larger and reproducible deviations, the institute's own calibration should be used and the manufacturer should be notified of the deviation. Note that, since the early nineties, there has been a known inconsistency of about $21 \%$ between calibrations by Amersham and NIST [70].

The non-uniformity $U_{F}$ of ophthalmic strontium sources should be below $30 \%$. $\left(U_{F}\right.$ determined with film for a concave source gives an overestimation of the true non-uniformity, therefore no action levels are presented for this case, although our experience with two concave sources suggests that the same levels for $U_{F}$ can be used as for planar sources). The source asymmetry $U_{A S}$ should be below $20 \%$.

Regarding the percentage depth dose no demands are made. Under normal circumstances no changes in these properties are expected. However, it is in the interest of the customer to know and, if possible, verify the depth dose characteristics of a source in case of changing circumstances. An example may be a change in the source design or the manufacturing process.

\section{Action levels for ophthalmic ruthenium sources}

The source strength $\dot{D}\left(r_{0}, z_{0}\right)$ of ruthenium sources should be within $\pm 10 \%$ of the value specified by the manufacturer, corrected for source decay. In the case of higher and reproducible deviations, the manufacturer should be contacted in order to resolve the cause of the discrepancy. It is advisable to ask the manufacturer for a review of the source calibration.

The non-uniformity $U_{F}$ of the ruthenium sources should be below $30 \%$ and the source asymmetry $U_{A S}$ should be below $20 \%$.

There may be reasons to deviate from the recommended values of non-uniformity and asymmetry, for example for asymmetrical sources with a higher dose rate close to the cutout. One may also argue that a high dose rate close to the edge of the source is desirable to obtain better depth dose characteristics. It is, however, important to keep in mind that too large differences in surface dose rates may cause other side-effects.

Regarding the percentage depth dose no demands are made. Under normal circumstances no changes in these properties are expected. However, it is in the interest of the user to know and, if possible, verify the depth dose characteristics of a source in case of for example a change in source design or manufacturing process.

Table 6-2 NCS recommendations on maximum deviations in source strength, maximum source nonuniformity and asymmetry.

\begin{tabular}{|c|c|c|c|}
\hline Technique & $\begin{array}{c}\text { Maximum deviation } \\
\text { in source strength }\end{array}$ & $\begin{array}{c}\text { Maximum source } \\
\text { non-uniformity } \boldsymbol{U}_{\boldsymbol{F}}\end{array}$ & $\begin{array}{c}\text { Maximum source } \\
\text { asymmetry } \boldsymbol{U}_{A S}\end{array}$ \\
\hline IVBT & $\pm 10 \%$ & $10 \%$ & n.a. \\
\hline Ophthalmic & $\pm 10 \%$ & $30 \%$ & $20 \%$ \\
\hline
\end{tabular}




\subsection{NCS recommendations on verification of physical parameters}

This section contains recommendations on several physical parameters to be verified as part of a quality control programme. The recommended frequency of these verifications is also given. This frequency is regarded as a minimal frequency of testing, in accordance with the recommendations in NCS report 13. The abbreviations for test frequencies in the following two sections are:

SE (each source exchange), P (each patient), iM (every i months), A (annually).

\subsubsection{Frequency of dosimetry}

\section{Overview of literature}

The frequencies for source strength measurements as recommended in the literature are given in Table 6-3. Some of the reports differentiate between sources with long and short half-lives.

Table 6-3 Recommended frequencies for source strength measurements in the literature.

\begin{tabular}{|l|l|l|}
\hline Report & Test frequency (short half-life & Test frequency (long half-life) \\
\hline AAPM TG56 (BT in general) & $3 \mathrm{M}$ & $3 \mathrm{M}$ \\
\hline ICRU ( $\beta$ and low energy $\mathrm{Y})$ & SE & SE + each few years \\
\hline DGMP 16 (IVBT) & SE & SE \\
\hline EVA GEC ESTRO (IVBT) & SE & SE + every year \\
\hline
\end{tabular}

The EVA GEC ESTRO working group advises daily source non-uniformity checks before application of stepping sources (probably because activity redistribution has been reported for early Guidant sources). A visual film check is sufficient, but should be further investigated with a densitometer in case of visible non-uniformity. Densitometric verification of source uniformity is recommended following every source exchange.

\section{NCS recommendation}

The NCS recommends that beta sources should at least be calibrated after every source exchange. The (relative) source strength of sources with a long period of use, such as the strontium eye applicators, should be verified at least every 3 years, because changes in the thickness of the filter may occur. In addition, the active source length, non-uniformity and (if applicable) asymmetry of beta sources should at least be verified after every source exchange.

\begin{tabular}{|c|c|}
\hline Test & Test frequency \\
\hline Source strength & SE or 3A whichever is shorter \\
\hline Active source length & SE \\
\hline Source non-uniformity & SE \\
\hline Source asymmetry (if applicable) & SE \\
\hline
\end{tabular}

\subsubsection{Source positioning (intravascular sources)}

If for intravascular brachytherapy sources a dummy source is available, the agreement between the positioning of the clinical and dummy source should be verified after a source exchange to avoid errors during treatment. This agreement should be better than $1 \mathrm{~mm}$, as larger differences may give rise to insufficient coverage of the lesion to be treated. The accuracy of stepping intravascular sources should also be verified visually. The agreement should be better than $1 \mathrm{~mm}$.

\footnotetext{
* Long or short is not specified in this report.
} 


\begin{tabular}{|c|c|}
\hline Test & Test frequency \\
\hline Source positioning (max. error is $1 \mathrm{~mm})$ & SE \\
\hline
\end{tabular}

\subsubsection{Irradiation timer}

No other considerations than for standard equipment are valid here. In some situations the source timer is replaced with the source and a higher frequency than once a year is necessary. If an integrating measurement is used to measure the source strength, both timer and dose rate are verified in one measurement.

\begin{tabular}{|c|c|}
\hline Test & Test frequency \\
\hline Timer accuracy and linearity (external timer) & A \\
\hline Timer accuracy and linearity (internal timer) & SE or A, whichever is shorter \\
\hline
\end{tabular}

\subsubsection{Treatment verification}

- Treatment time calculation should be checked independently before each treatment.

- Treatment time should be monitored with two independent timers. One additional timer should be used if the automated device contains one timer.

- Independent registration of the treatment time and absorbed dose should be carried out.

\subsection{NCS recommendations on verification of safety aspects}

This section contains recommendations on several safety aspects in a quality control programme.

\subsubsection{Source monitoring}

During and after application of intravascular or ruthenium sources two types of monitoring should be performed.

1) The radiation level outside the body should be verified during clinical application of the source.

2) Removal of the source should be verified after treatment.

Verification should be performed with a standard background radiation monitor. This is not necessary for the ophthalmic strontium applicators because of the short radiation time and because the source is not inside the body.

\subsubsection{Interlocks (intravascular sources)}

Any available facility for non-standard source retraction of intravascular sources should be tested on a regular basis. Because mechanical parts are sometimes exchanged with the source, the frequency of this check should be SE. For long-lived isotopes, this should be at least 4M. All currently available afterloaders block source ejection when a catheter is not attached. This function should be tested with the same frequency. Every catheter should be checked for obstructions with a dummy source before a clinical source is ejected.

\begin{tabular}{|c|c|}
\hline Test & Test frequency \\
\hline $\begin{array}{c}\text { Emergency source retraction system } \\
\text { (interrupt button, power backup, } \\
\text { manual retraction facility) }\end{array}$ & SE or 4M, whichever is shorter \\
\hline Missing catheter & SE or 4M, whichever is shorter \\
\hline Catheter obstruction & $\mathrm{P}$ \\
\hline
\end{tabular}

\subsubsection{Radiation safety}

The leakage radiation level at a fixed distance from the source holder or container should be measured after source exchange. For long-lived isotopes this should be repeated annually. 
The integrity of intravascular sources should be verified by checking the clinical catheters after the treatment using a contamination monitor. In case of the Novoste sources the water should also be checked for contamination, both for dosimetry procedures and clinical application of the source

The integrity of ophthalmic sources should be verified with a wipe test after every treatment.

\begin{tabular}{|c|c|}
\hline Test & Test frequency \\
\hline Leakage radiation & SE or A, whichever is shorter \\
\hline Source integrity & $\mathrm{P}$ \\
\hline
\end{tabular}

\subsubsection{Integrity of catheter (intravascular sources)}

The integrity (for example kinking) of every catheter should be checked visually and with a dummy source before each treatment. See also Section 6.6.2.

\begin{tabular}{|c|c|}
\hline Test & Test frequency \\
\hline Catheter integrity & $\mathrm{P}$ \\
\hline
\end{tabular}

\subsubsection{Emergency aspects}

No other considerations than for standard equipment are applicable here.

\begin{tabular}{|c|c|}
\hline Test & Test frequency \\
\hline Emergency equipment functionality & $4 \mathrm{M}$ \\
\hline Training of emergency procedures & $\mathrm{A}$ \\
\hline
\end{tabular}

As an example, for intravascular sources an emergency storage container and tweezers or pliers should be available in case the source does not retract and the catheter has to be removed.

\subsection{Communication of QC results to clinician}

When using sources that are frequently replaced, the clinician should be aware of the nominal dose distribution of the type(s) of source being used. In the case of sources used for a period of one year or longer, the clinician should be aware of the dose distribution of each individual source. The tolerance levels used for source strength, non-uniformity and asymmetry should be known to the clinician. 


\section{Summary of NCS recommendations}

The detectors that are suitable for strength and uniformity measurements (both $U_{F}$ and $U_{A S}$ ) are given in Table 7-1.

Table 7-1. Suitable detectors for determination of strength and uniformity of sealed beta sources.

\begin{tabular}{|c|c|c|c|c|c|c|c|c|c|}
\hline & & $\begin{array}{c}\text { Plastic } \\
\text { scintillator }\end{array}$ & $\begin{array}{c}\text { Well-type } \\
\text { ionization } \\
\text { chamber }\end{array}$ & $\begin{array}{l}\text { HD810 } \\
\text { film }\end{array}$ & $\begin{array}{l}\text { MD55-2 } \\
\text { film }\end{array}$ & $\begin{array}{l}\text { HS } \\
\text { film }\end{array}$ & Diode & $\begin{array}{c}\text { Plane- } \\
\text { parallel } \\
\text { ionization } \\
\text { chamber }\end{array}$ & $\begin{array}{c}\text { Extrapolation } \\
\text { chamber at } \\
\text { standards } \\
\text { institute }\end{array}$ \\
\hline \multirow{3}{*}{$\begin{array}{c}\text { Intravasc. } \\
\text { BT }\end{array}$} & $S S$ & $\mathrm{X}$ & $\mathrm{X}$ & & & & & & \\
\hline & $U_{F}$ & $X$ & & $X$ & $x$ & $x$ & & & \\
\hline & $P D D$ & $X$ & & $X$ & $x$ & $x$ & & & \\
\hline \multirow{4}{*}{$\begin{array}{c}\text { Ophthalmic } \\
\mathrm{Sr}\end{array}$} & $S S$ & $\bar{X}$ & & & & & & $X^{*}$ & $\mathrm{X}$ \\
\hline & $U_{F}$ & & & $X$ & $x$ & $x$ & & & \\
\hline & $U_{A S}$ & & & $X$ & $x$ & $x$ & & & \\
\hline & $P D D$ & $X$ & & $X$ & $X$ & $x$ & & & \\
\hline \multirow{4}{*}{$\begin{array}{c}\text { Ophthalmic } \\
\text { Ru }\end{array}$} & $S S$ & $\bar{X}$ & & & & & & & \\
\hline & $U_{F}$ & $X$ & & & & & $X$ & & \\
\hline & $U_{A S}$ & $X$ & & $x$ & $x$ & $x$ & $X$ & & \\
\hline & $P D D$ & $X$ & & $x$ & $X$ & $x$ & $X$ & & \\
\hline
\end{tabular}

$S S=$ Source strength measurement; $U_{F}=$ uniformity (flatness); $U_{A S}=$ asymmetry; $P D D=$ Percentage Depth Dose; $X=$ suitable detector; $x=$ other possible detector.

The reference points that are recommended for strength measurements on beta sources are given in Table 7-2.

Table 7-2 Reference points or reference distance for the 3 groups of beta sources.

\begin{tabular}{|c|c|c|c|}
\hline & $\begin{array}{c}\text { Intravascular } \\
\text { sources }\end{array}$ & $\begin{array}{c}\text { Planar ophthalmic } \\
\text { sources }\end{array}$ & $\begin{array}{c}\text { Concave ophthalmic } \\
\text { sources }\end{array}$ \\
\hline$r_{0}(\mathbf{m m}$ in water) & 2 & 0 & 0 \\
\hline $\mathrm{z}_{0}(\mathbf{m m}$ in water) & & 2 & 2 \\
\hline
\end{tabular}

The NCS recommends to keep the relative standard uncertainty of the detectors below $4 \%$ $(1 \sigma)$ for source strength, non-uniformity and asymmetry. In order to do so, the measurement system should be tested in terms of e.g. reproducibility and mechanical tolerances. The action levels for deviations in strength and the action levels for non-uniformity and asymmetry are given in Table 7-3.

Table 7-3 NCS recommendations on maximum deviations in source strength, maximum source nonuniformity and asymmetry

\begin{tabular}{|c|c|c|c|}
\hline Technique & $\begin{array}{c}\text { Maximum deviation } \\
\text { in source strength }\end{array}$ & $\begin{array}{c}\text { Maximum source } \\
\text { non-uniformity } \boldsymbol{U}_{\boldsymbol{F}}\end{array}$ & $\begin{array}{c}\text { Maximum source } \\
\text { asymmetry } \boldsymbol{U}_{\boldsymbol{A S}}\end{array}$ \\
\hline IVBT & $\pm 10 \%$ & $10 \%$ & n.a. \\
\hline Ophthalmic Sr or $\mathrm{Ru}$ & $\pm 10 \%$ & $30 \%$ & $20 \%$ \\
\hline
\end{tabular}

\footnotetext{
* Relative source strength measurements on one source (type), planar sources only.
} 
An overview of the recommended QC on physical and safety aspects is given below. The recommended test frequency is given in the right column.

\begin{tabular}{|c|c|}
\hline Test & Test frequency \\
\hline Source strength & SE or 3A whichever is shorter \\
\hline Active source length & $\mathrm{SE}$ \\
\hline Source non-uniformity & $\mathrm{SE}$ \\
\hline Source asymmetry (if applicable) & $\mathrm{SE}$ \\
\hline Source positioning (max. error is 1 mm) & $\mathrm{SE}$ \\
\hline Timer accuracy and linearity (external timer) & $\mathrm{A}$ \\
\hline Timer accuracy and linearity (internal timer) & SE or A, whichever is shorter \\
\hline $\begin{array}{c}\text { Emergency source retraction system } \\
\text { (interrupt button, power backup, } \\
\text { manual retraction facility) }\end{array}$ & $\mathrm{SE}$ or 4M, whichever is shorter \\
\hline Missing catheter & $\mathrm{SE}$ or 4M, whichever is shorter \\
\hline Catheter obstruction & $\mathrm{P}$ \\
\hline Leakage radiation & $\mathrm{SE}$ or A, whichever is shorter \\
\hline Source integrity & $\mathrm{P}$ \\
\hline Catheter integrity & $\mathrm{P}$ \\
\hline Emergency equipment functionality & $\mathrm{A}$ \\
\hline Training of emergency procedures & \\
\hline
\end{tabular}




\section{Appendix 1. Nuclear data of relevant beta emitters}

Table A1 List of nuclear data of the isotopes that may be used for beta sources.

\begin{tabular}{|c|c|c|c|c|c|c|}
\hline $\mathbf{Z}$ & $\mathbf{A}$ & element & decay & $E_{\max }(k e V)$ & $E_{a v}(k e V)$ & $T_{1 / 2}$ \\
\hline 15 & 32 & $P$ & $\beta^{-}$ & 1710.6 & 694.7 & $14.262 \mathrm{~d}$ \\
\hline 38 & 90 & Y & $\beta^{-}$ & 2282.0 & 934.8 & $64.10 \mathrm{~h}$ \\
\hline 39 & 90 & $\mathrm{Sr}$ & $\beta^{-}$ & 546.2 & 195.7 & 28.78 y \\
\hline 44 & 106 & $\mathrm{Ru}$ & $\beta^{-}$ & 39.4 & 10.03 & $373.59 \mathrm{~d}$ \\
\hline 45 & 106 & $\mathrm{Rh}$ & $\beta^{-}$ & 3541 (79\%) & 1509 & $29.80 \mathrm{~s}$ \\
\hline & & & $\gamma$ & \multicolumn{2}{|c|}{$\begin{array}{c}622(10 \%) \text { and } \\
512(21 \%) \\
\end{array}$} & \\
\hline \multirow[t]{2}{*}{58} & 144 & $\mathrm{Ce}$ & $\beta^{-}$ & 318.7 (77\%) & 91 & $284.893 \mathrm{~d}$ \\
\hline & & & $\gamma$ & \multicolumn{2}{|c|}{$134(11 \%)$} & \\
\hline \multirow[t]{2}{*}{59} & 144 & $\operatorname{Pr}$ & $\beta^{-}$ & $2997.5(98 \%)$ & 1221 & $17.28 \mathrm{~min}$ \\
\hline & & & $\gamma$ & \multicolumn{2}{|c|}{$\begin{array}{c}2186(0.7 \%) \text { and } \\
696(1.5 \%) \\
\end{array}$} & \\
\hline \multirow[t]{2}{*}{74} & 188 & W & $\beta^{-}$ & $349(99 \%)$ & 100 & $69.4 \mathrm{~d}$ \\
\hline & & & $\gamma$ & \multicolumn{2}{|c|}{$291(0.4 \%)$} & \\
\hline \multirow[t]{2}{*}{75} & 188 & $\mathrm{Re}$ & $\beta^{-}$ & $2120.4(71 \%)$ & 795 & $16.98 \mathrm{~h}$ \\
\hline & & & $\gamma$ & \multicolumn{2}{|c|}{$\begin{array}{c}155(15 \%) \\
\text { and } 1134(0.7 \%)\end{array}$} & \\
\hline
\end{tabular}

$Z=$ atomic number, $A$ = mass number, $E_{\max }=$ maximum beta energy, $E_{a v}=$ average beta energy and $T_{1 / 2}=$ half-life.

\section{Sources:}

- Table of Isotopes, Richard B. Firestone [77].

- \% values, gamma energies and average values are taken from Raddecay version 2 , using US Department of Energy decay data [78].

\footnotetext{
* Most important contributions are given. For the sources that emit both betas and gammas the most important contributions are given.
} 


\section{Appendix 2. Specifications of beta sources}

\section{Appendix 2.1 Specifications of intravascular sources}

Table A2 Source specifications of intravascular sources (Guidant and Novoste).

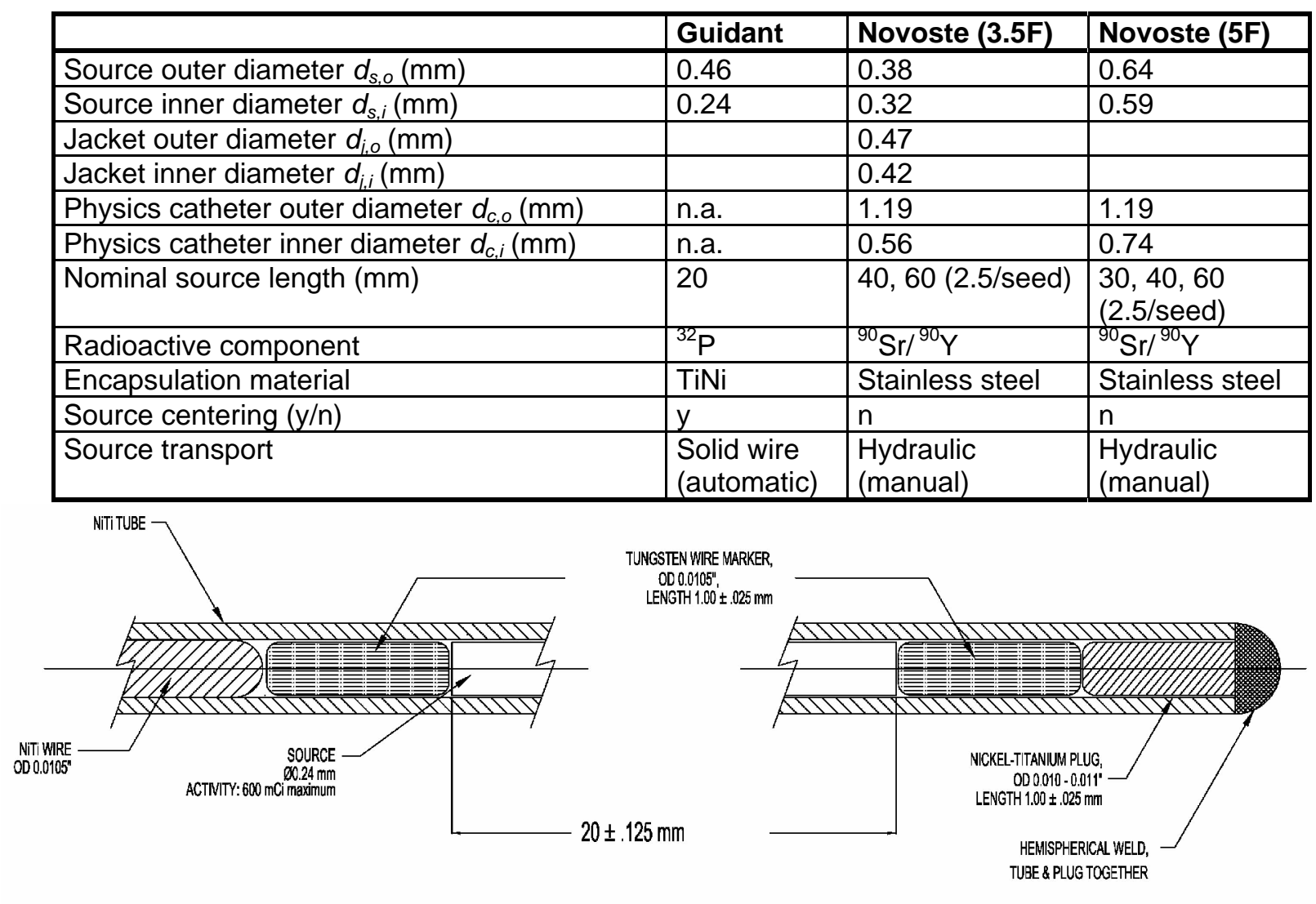

Figure A1 Schematic view of the Guidant $20 \mathrm{~mm}$ source

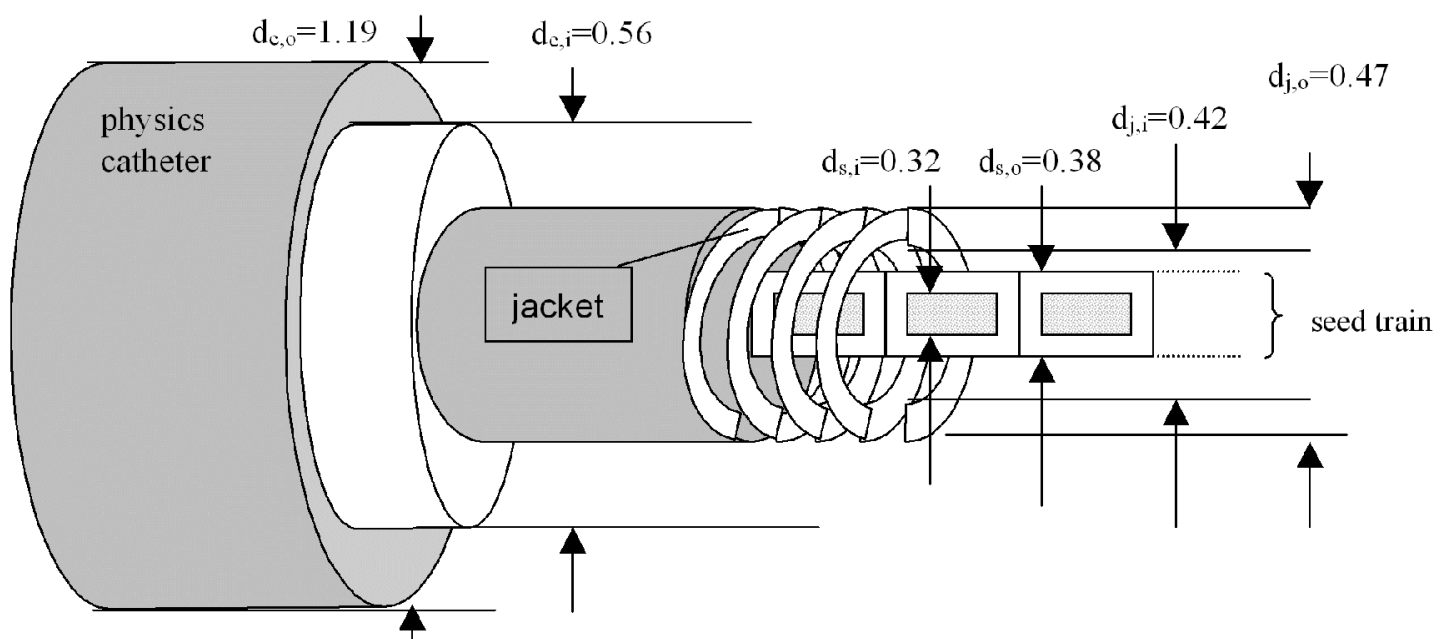

Figure A2 Schematic view of the Novoste 3.5F jacketed source in the physics catheter. $d_{c, 0}=$ outer diameter of physics catheter, $d_{c, i}=$ inner diameter of physics catheter, $d_{j, o}=$ outer diameter of jacket, $d_{j, i}=$ inner diameter of jacket, $d_{s, o}=$ outer diameter of seed, $d_{s, i}=$ inner diameter of seed encapsulation. All sizes are in $\mathrm{mm}$. 
Table A3 Relative depth dose data of intravascular sources: Guidant $(20 \mathrm{~mm})$, Novoste 3.5F and 5F (both $40 \mathrm{~mm}$ long sources).

Column 2: Depth dose data from manual Guidant Galileo afterloader (20 $\mathrm{mm}$ source)

Columns 3 and 4: Fits on experimental data from Weber et al, Novoste (personal communication).

\begin{tabular}{|c|c|c|c|}
\hline Distance & $\underset{{ }^{32} \mathrm{P}}{\text { Guidant } 20 \mathrm{~mm}}$ & $\begin{array}{c}\text { Novoste } 40 \mathrm{~mm} \\
5 \mathrm{~F}{ }^{90} \mathrm{Sr} /{ }^{90} \mathrm{Y} \\
\end{array}$ & \begin{tabular}{|c|} 
Novoste $40 \mathrm{~mm}$ \\
$3.5 \mathrm{~F}{ }^{90} \mathrm{Sr} /{ }^{\circ} \mathrm{Y}$ \\
\end{tabular} \\
\hline$r(\mathrm{~mm})$ & $\mathrm{D}_{\mathrm{w}}(\%)$ & $\mathrm{D}_{\mathrm{w}}(\%)$ & $\mathrm{D}_{\mathrm{w}}(\%)$ \\
\hline 0.50 & 698 & & \\
\hline 0.75 & 449 & & \\
\hline 1.00 & 315 & & \\
\hline 1.25 & 231 & & \\
\hline 1.50 & 173 & & \\
\hline 1.61 & - & 136 & 136 \\
\hline 1.75 & 131 & 120 & 121 \\
\hline 2.00 & 100 & 100 & 100 \\
\hline 2.25 & 76.4 & 83.8 & 83.6 \\
\hline 2.50 & 58.1 & 70.1 & 69.9 \\
\hline 2.75 & 44.0 & 58.7 & 58.5 \\
\hline 3.00 & 33.0 & 49.1 & 48.9 \\
\hline 3.25 & 24.5 & 41.0 & 40.8 \\
\hline 3.50 & 18.0 & 34.3 & 34.1 \\
\hline 3.75 & 13.0 & 28.6 & 28.4 \\
\hline 4.00 & 9.1 & 23.8 & 23.7 \\
\hline 4.25 & 6.3 & 19.8 & 19.7 \\
\hline 4.50 & 4.3 & 16.5 & 16.3 \\
\hline 4.75 & & 13.7 & 13.5 \\
\hline 5.00 & & 11.3 & 11.2 \\
\hline
\end{tabular}
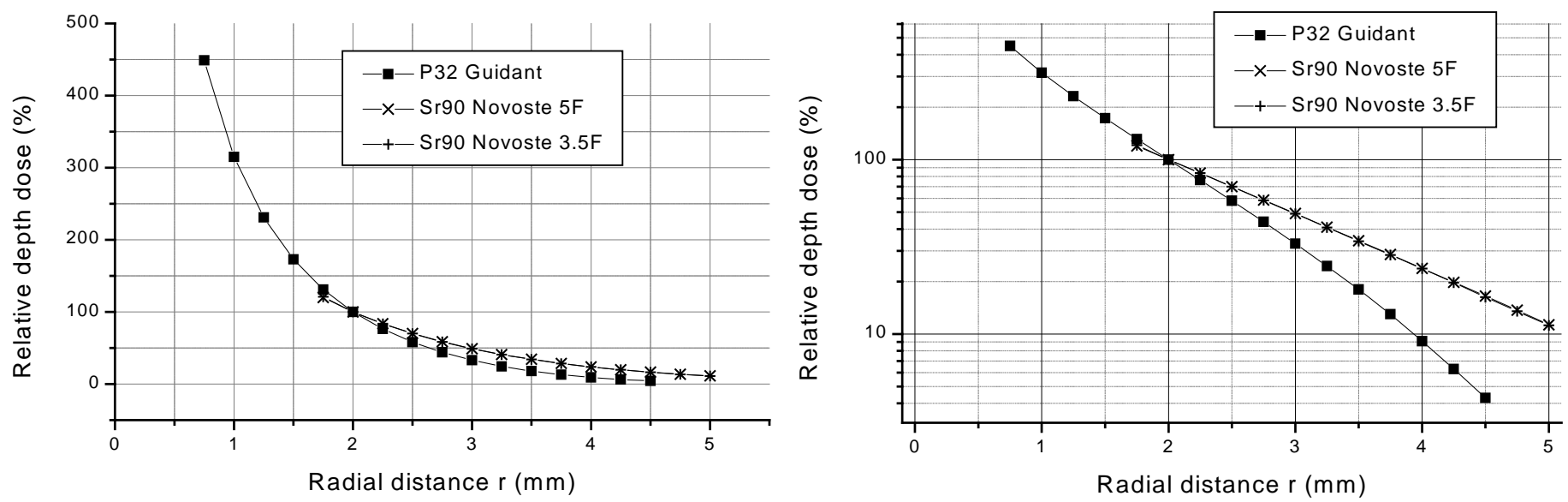

Figure A3 Percentage depth dose for ${ }^{32} \mathrm{P}$ Guidant sources and ${ }^{90} \mathrm{Sr}^{90} \mathrm{Y}$ Novoste sources (3.5F and $5 \mathrm{~F}$ ) according to data in Table A3. 
Appendix 2.2 Specifications of ophthalmic ruthenium sources

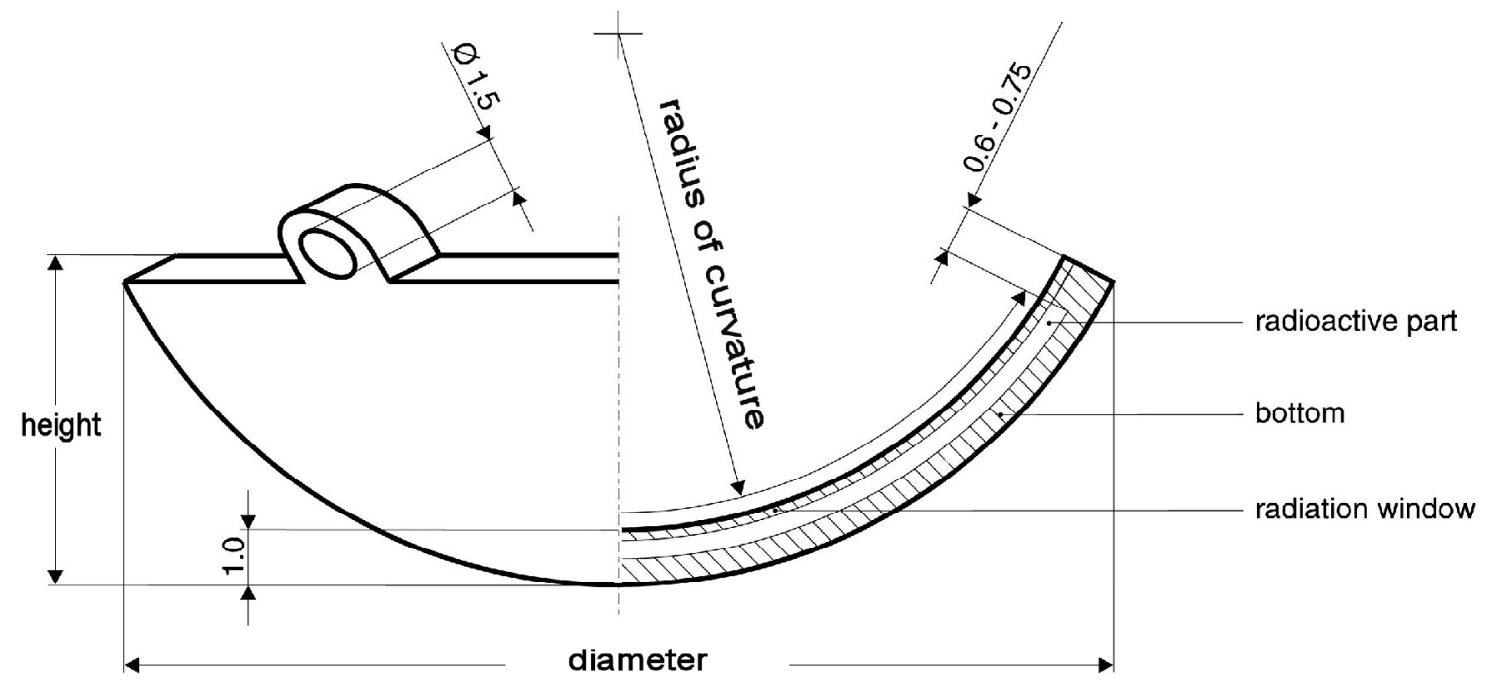

Figure A4 Cross-section of the ruthenium plaque. Typical source parameters diameter, height and radius of curvature are depicted.

Table A4 Source parameters of available ruthenium plaques (BEBIG, Berlin, Germany, March 2003).

\begin{tabular}{|c|c|c|c|}
\hline Type & $\begin{array}{c}\text { Diameter } \\
(\mathbf{m m})\end{array}$ & $\begin{array}{c}\text { Height } \\
(\mathbf{m m})\end{array}$ & $\begin{array}{c}\text { Radius of } \\
\text { curvature } \\
(\mathbf{m m})\end{array}$ \\
\hline CCZ & 11.6 & 2.3 & 12 \\
\hline CCY & 11.6 & 2.3 & 12 \\
\hline CCX & 11.6 & 2.3 & 12 \\
\hline CXS & $\begin{array}{c}11.6 \\
(7.7 \text { active })\end{array}$ & 2.3 & 12 \\
\hline CCA & 15.3 & 3.3 & 12 \\
\hline CCD & 17.9 & 4.3 & 12 \\
\hline CCB & 20.2 & 5.5 & 12 \\
\hline CGD & 22.3 & 6.1 & 13 \\
\hline CCC & 24.8 & 8.0 & 13 \\
\hline COB & 19.8 & 5.2 & 12 \\
\hline COD & 25.4 & 7.5 & 14 \\
\hline COE & 19.8 & 5.2 & 12 \\
\hline COC & 25.4 & 7.5 & 14 \\
\hline CIA & 15.3 & 3.3 & 12 \\
\hline CIB & 20.2 & 5.4 & 12 \\
\hline CIB-2 & 20.2 & 5.4 & 12 \\
\hline
\end{tabular}

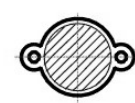

CCZ

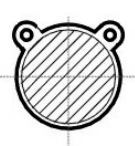

CCA

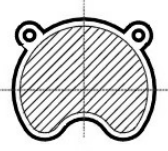

$C O B$

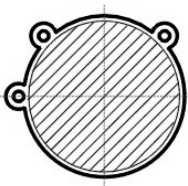

CGD

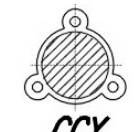

$\mathrm{CCY}$

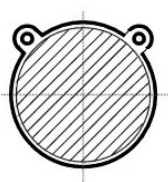

$C C B$

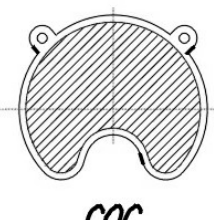

$\mathrm{coc}$

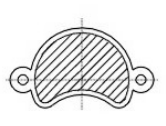

$C A$

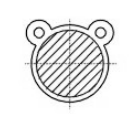

cCX

CXS

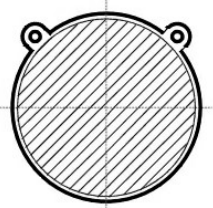

CCC

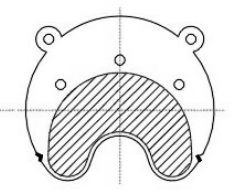

$C O D$<smiles></smiles>

CCD
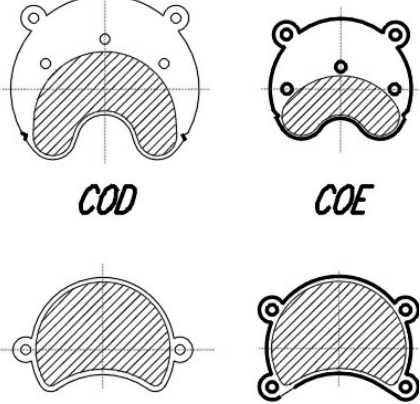

$C / B$<smiles>C1=C2CCCC(C2)O1</smiles>

CIB2

Figure A5 Plane view of the sources described in Table A4. CIA, CIB, CIB-2 are plaques with an adaptation for the iris; $C O B, C O C, C O D$ and $C O E$ have an adaptation for the optical nerve. 


\section{Appendix 2.3 Depth dose of Amersham strontium sources}

Table A5 Percentage depth dose table for SIA.20 and SIA.6 Amersham ${ }^{90} \mathrm{Sr}^{90} \mathrm{Y}$ sources normalized to $100 \%$ at a depth of $2 \mathrm{~mm}$ in solid water (RMI-457). Fitted values to the experimental curves of this report are given as well as specifications of the SIA.20 as provided by Amersham. The depth dose values are given as absorbed dose to solid water. The difference between these values and the absorbed dose to water is expected to be small as illustrated in Appendix 3.

\begin{tabular}{|c|c|c|c|}
\hline $\begin{array}{c}\mathbf{Z} \\
(\mathbf{m m})\end{array}$ & $\begin{array}{c}\text { SIA.20 (fit) } \\
(\%)\end{array}$ & $\begin{array}{c}\text { Amersham } \\
\text { SIA.20 } \\
\text { specs }\end{array}$ & $\begin{array}{c}\text { SIA.6 (fit) } \\
(\%)\end{array}$ \\
\hline 0 & 325 & 250 & 177 \\
\hline 0.25 & 281 & & 171 \\
\hline 0.50 & 243 & & 163 \\
\hline 0.75 & 210 & & 153 \\
\hline 1.00 & 181 & 175 & 143 \\
\hline 1.25 & 156 & & 132 \\
\hline 1.50 & 135 & & 121 \\
\hline 1.75 & 116 & & 110 \\
\hline $\mathbf{2 . 0 0}$ & $\mathbf{1 0 0}$ & $\mathbf{1 0 0}$ & $\mathbf{1 0 0}$ \\
\hline 2.25 & 86 & & 90.0 \\
\hline 2.50 & 73.9 & & 79.9 \\
\hline 2.75 & 63.4 & & 70.0 \\
\hline 3.00 & 54.3 & 50 & 60.8 \\
\hline 3.25 & 46.5 & & 52.0 \\
\hline 3.50 & 39.6 & & 43.4 \\
\hline 3.75 & 33.7 & & 35.5 \\
\hline 4.00 & 28.6 & & 28.9 \\
\hline 4.25 & 24.2 & & 23.0 \\
\hline 4.50 & 20.3 & & 18.0 \\
\hline 4.75 & 17.0 & & 14.0 \\
\hline 5.00 & 14.1 & & 10.9 \\
\hline
\end{tabular}
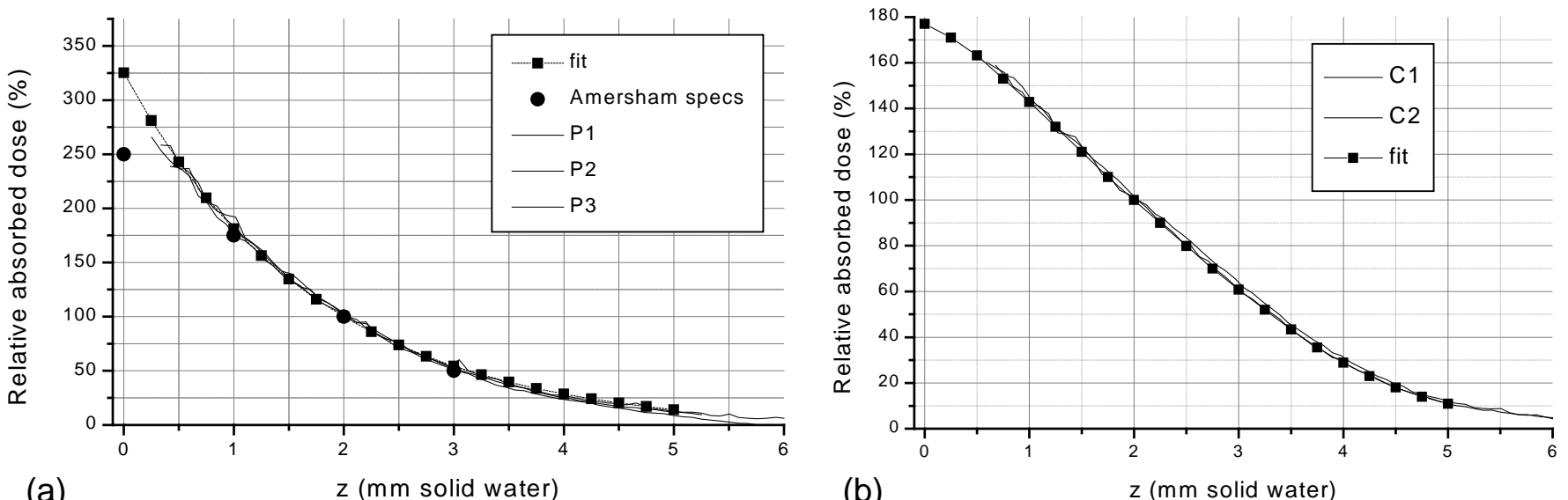

(a)

(b)

$\mathrm{z}$ ( $\mathrm{mm}$ solid water)

Figure A6 Percentage depth dose curves for Amersham ${ }^{90} \mathrm{Sr}^{90} \mathrm{Y}$ sources investigated in this report: a) SIA.20 sources (fitted value, manufacturers specs, measured curve with HD810 on P1, P2, P3)

b) SIA.6 sources (fitted value, measured curve with HS on C1 and C2) 


\section{Appendix 3. Scaling from water equivalent plastic to water}

For point source geometries the absorbed dose $D_{m}$ in a medium $(\mathrm{m})$ relative to water $(\mathrm{w})$ can be calculated through Equation A-1 [71].

$$
D_{m}(r)=\eta_{m, w}{ }^{3} \frac{\rho_{m}{ }^{2}}{\rho_{w}{ }^{2}} D_{w}\left(\eta_{m, w} \frac{\rho_{m}}{\rho_{w}} r\right)
$$

In this equation $\eta_{m, w}$ is the scaling factor or relative attenuation of medium $(\mathrm{m})$ relative to water. The absorbed dose to the material $(\mathrm{m})$ is also dependent on the ratio of the densities $\rho_{w}$ and $\rho_{m}$. An overview of the most relevant scaling factors and densities is given in Table A6.

An example of scaling is found in Figure A7, where a depth dose curve for an SIA.20 strontium source is depicted. From the original absorbed dose curve in solid water (RMI457), curves in both water and other materials are calculated using Equation 3-11. By applying this equation the assumption is made that the point source approximation is sufficient. The differences between water, solid water and polystyrene are relatively small (below 1\% in this region), but for PMMA and A150 the difference is larger.

Table A6 Scaling parameters for some of the most frequently used water equivalent materials [3].

\begin{tabular}{|l|l|l|}
\hline $\begin{array}{l}\text { Plastic } \\
\text { (material } \mathbf{~ m})\end{array}$ & $\begin{array}{l}\text { Density } \rho_{\boldsymbol{m}} \\
\left(\mathbf{g ~ c m}^{-3}\right)\end{array}$ & $\begin{array}{l}\text { Scaling factor } \eta_{\boldsymbol{m}, \mathbf{w}} \\
\text { relative to water }\end{array}$ \\
\hline $\begin{array}{l}\text { WT-1 } \\
\text { (solid water) }\end{array}$ & 1.020 & 0.957 \\
\hline $\begin{array}{l}\text { RMl-457 } \\
\text { (solid water) }\end{array}$ & 1.030 & 0.957 \\
\hline A-150 & 1.127 & 0.968 \\
\hline PMMA & 1.19 & 0.949 \\
\hline Polystyrene & 1.05 & 0.938 \\
\hline
\end{tabular}
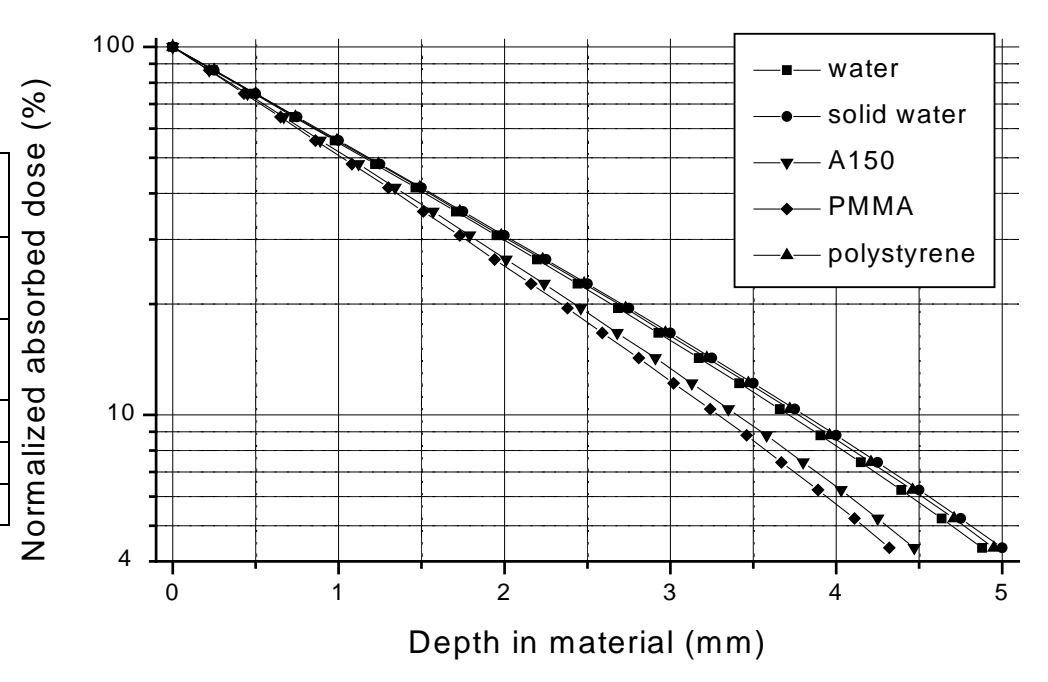

Figure A7 PDDs in several materials based on an experimental curve in solid water (RMI-457) and calculated with Equation A-1.

For a cylindrical source with an outer radius $r_{s}$ the absorbed dose to a medium $\mathrm{m}$ can be expressed according to Equation A-2. This equation is only valid for sources longer than twice the beta particle range [72].

$$
r D_{m}(r)=\eta_{m, w}\left[r_{s}+\eta_{m, w} \frac{\rho_{m}}{\rho_{w}}\left(r-r_{s}\right)\right] D_{w}\left[r_{s}+\eta_{m, w} \frac{\rho_{m}}{\rho_{w}}\left(r-r_{s}\right)\right]
$$

More detail on the theory of scaling can be found in other publications $[65,72]$. The results obtained with scaling are dependent on energy, but usually accurate to within $\sim 3 \%$ [72].

\footnotetext{
"WT-1, Solid Water ${ }^{\mathrm{TM}}$, Gammex-RMI (Middleton, WI, USA).

${ }^{\dagger}$ RMI-457, Solid Water ${ }^{T M}$, Gammex-RMI (Middleton, WI, USA). The scaling factor of WT-1 is used. In [79] a different scaling factor of 0.949 is given, but equal for both types of solid water.

${ }^{7}$ A-150 plastic, Exradin (Lisle, IL, USA).
} 


\section{Appendix 4. Detector properties}

Table A7 Types and serial numbers of detectors used for experiments

\begin{tabular}{|c|c|c|c|c|}
\hline Detector & Company & $\begin{array}{l}\text { Manufacturer } \\
\text { ID }\end{array}$ & SN & Insert REF \\
\hline $\begin{array}{l}\text { HDR1000 Plus well-type ionization } \\
\text { chamber }\end{array}$ & $\begin{array}{l}\text { Standard } \\
\text { Imaging }\end{array}$ & 90008 & A002855 & 70028 \\
\hline IVB1000 well-type ionization chamber & $\begin{array}{l}\text { Standard } \\
\text { Imaging }\end{array}$ & 9009 & $\mathrm{H} 011011$ & 70036 \\
\hline Optidos plastic scintillator & PTW & T10013 & 0050 & \\
\hline Optidos photomultiplier & PTW & 60006 & 0062 & \\
\hline $\begin{array}{l}\text { (Radioactive check device } \\
\left.33.3 \mathrm{MBq}{ }^{90} \mathrm{Sr} /{ }^{90} \mathrm{Y}\right)\end{array}$ & (PTW) & (T8921/8922) & $(1679)$ & \\
\hline GAFChromic HD810 & ISP & $\begin{array}{c}\text { 600-27726-03- } \\
\text { SA2A10 }\end{array}$ & $\mathrm{J} 1235 \mathrm{H} 810$ & \\
\hline GAFChromic HS & ISP & $37-044-0000$ & $\begin{array}{c}\text { I0144HS } \\
\text { (K0223HS) }\end{array}$ & \\
\hline GafChromic MD55-2 & ISP & $37-041$ & J1548MD55 & \\
\hline Markus 23343 ionization chamber & PTW & TW23343 & 3344 & \\
\hline EFD electron semiconductor detector & Scanditronix & $\mathrm{EFD}^{3 \mathrm{G}}$ & DEB0023094 & \\
\hline Excalibur CDX 2000A electrometer & $\begin{array}{l}\text { Standard } \\
\text { Imaging }\end{array}$ & $90003 A$ & B001817 & \\
\hline
\end{tabular}

Table A8 Active volume and length of sweet spot of well-type ionization chambers (manufacturers specifications).

\begin{tabular}{|l|c|c|}
\hline Well-type ionization chamber & $\begin{array}{c}\text { Active volume } \\
\mathbf{( c m}^{\mathbf{3}} \mathbf{)}\end{array}$ & $\begin{array}{c}\text { Length of sweet spot } \\
(\mathbf{m m} \text { ) for which difference } \\
\text { in current }<\mathbf{0 . 5 \%}\end{array}$ \\
\hline HDR1000 Plus & 245 & 25 \\
\hline IVB1000 & 475 & 100 \\
\hline
\end{tabular}

Table A9 Size of detector and active volume for point like detectors (manufacturers specifications).

\begin{tabular}{|l|c|c|c|c|}
\hline Detector type & $\begin{array}{c}\text { Sensitive } \\
\text { diameter }(\mathbf{m m})\end{array}$ & $\begin{array}{c}\text { Outer } \\
\text { diameter } \\
(\mathbf{m m})\end{array}$ & $\begin{array}{c}\text { Offset depth } \\
\mathbf{( \mathbf { m m } )}\end{array}$ & $\begin{array}{c}\text { Sensitive } \\
\text { thickness } \\
(\mathbf{m m})\end{array}$ \\
\hline Optidos plastic scintillator & 1.0 & 5.2 & 0.35 & 1.0 \\
\hline Markus 23343 ionization chamber & 5.3 & & & 2.0 \\
\hline $\begin{array}{l}\text { EFD electron semiconductor } \\
\text { detector }\end{array}$ & 2.0 & 7.2 & 0.41 & 0.06 \\
\hline
\end{tabular}

Table A10 Dose range, thickness of film and sensitive layer for different types of radiochromic film.

\begin{tabular}{|l|c|c|c|}
\hline $\begin{array}{l}\text { Radiochromic film } \\
\text { type }\end{array}$ & $\begin{array}{c}\text { Dose } \\
\text { range } \\
(\mathbf{G y})\end{array}$ & $\begin{array}{c}\text { Film } \\
\text { thickness } \\
(\mu \mathbf{m})\end{array}$ & $\begin{array}{c}\text { Thickness of } \\
\text { sensitive layer } \\
(\mu \mathbf{m})\end{array}$ \\
\hline MD55-2 & $3-100$ & 278 & $2 \times 15$ \\
\hline HD810 & $50-2500$ & 107 & 7 \\
\hline HS & $0.5-40$ & 230 & 40 \\
\hline
\end{tabular}

\footnotetext{
"Distance from the detector surface to the edge of the sensitive volume.
} 


\section{Appendix 5. Plastic scintillator phantom for intravascular sources}

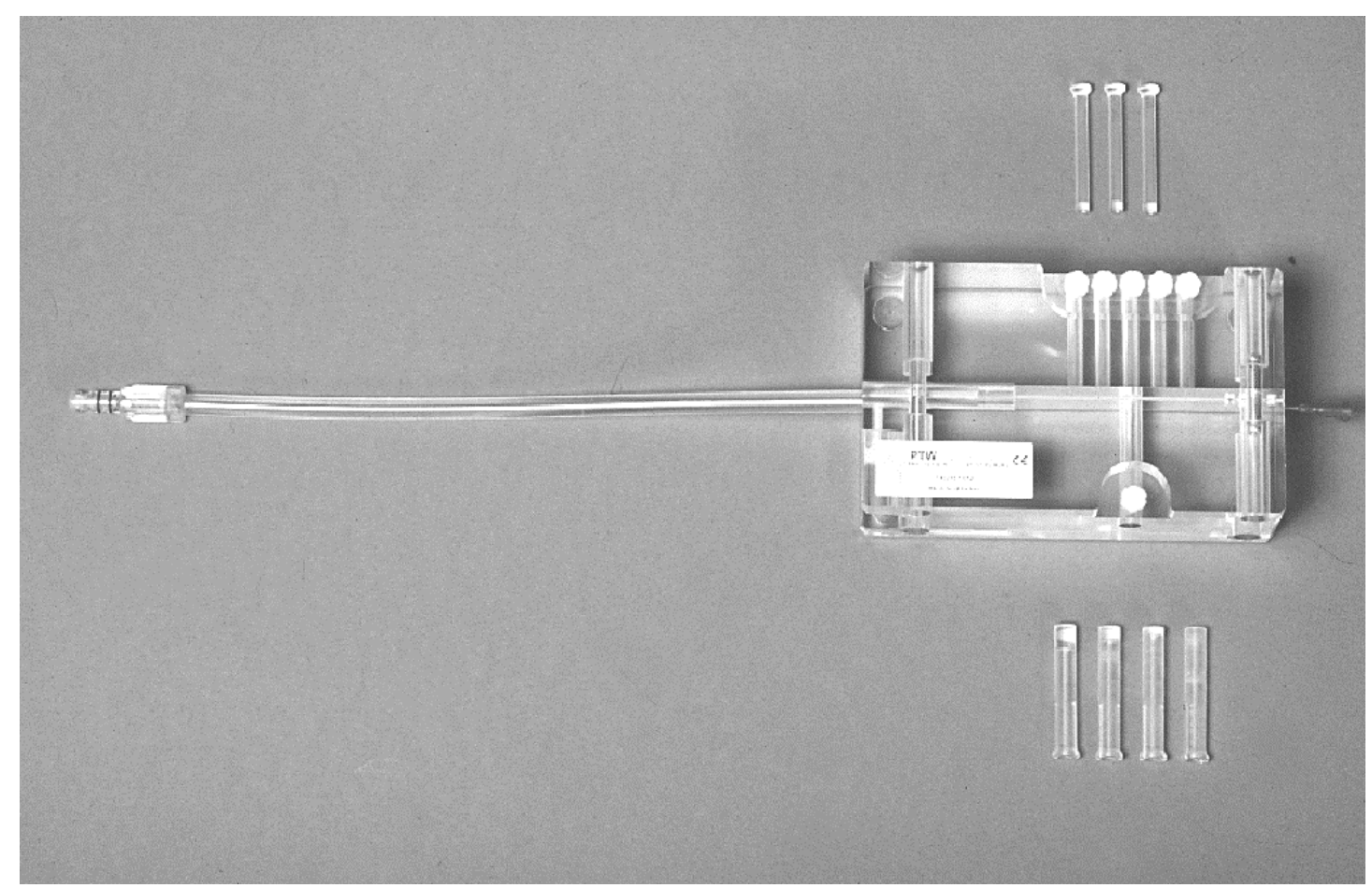

Figure A8 PTW phantom for plastic scintillator measurements on Novoste ${ }^{90} \mathrm{Sr}^{90} \mathrm{Y}$ source trains. The phantom for the Novoste sources has different plugs along the source axis at $z=-20,-10,0,10$ and $20 \mathrm{~mm}$. The phantom for the Guidant sources only has spacers at $z=0$. In the figure the Novoste physics catheter is also displayed.

Table A11 Measurement depth for plastic scintillator phantoms PTW for intravascular sources (corrected $\mathrm{mm}$ water).

\begin{tabular}{|c|c|}
\hline Guidant sources & Novoste sources \\
\hline 2.0 & 2 \\
\hline 2.6 & 5 \\
\hline 3.3 & 8 \\
\hline 10 & 12 \\
\hline
\end{tabular}




\section{Appendix 6. Technical drawing of film phantom for intravascular sources}

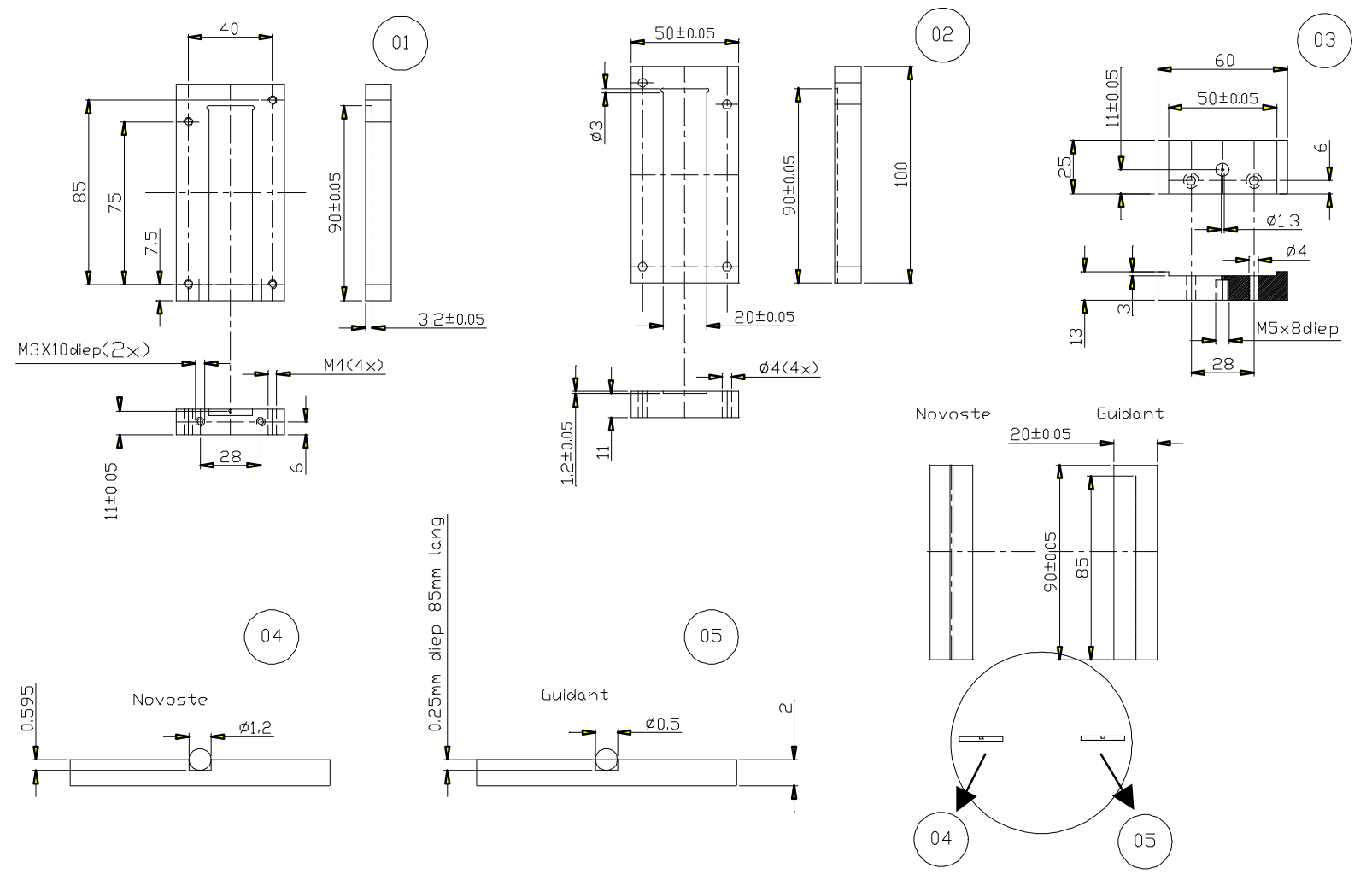

Figure A9 Design of film phantom. Part 1: bottom of phantom; part 2: top of phantom; part 3: phantom add-on for Guidant physics coupler connection; part 4: Novoste insert and part 5: Guidant insert (all sizes in $\mathrm{mm}$ ).

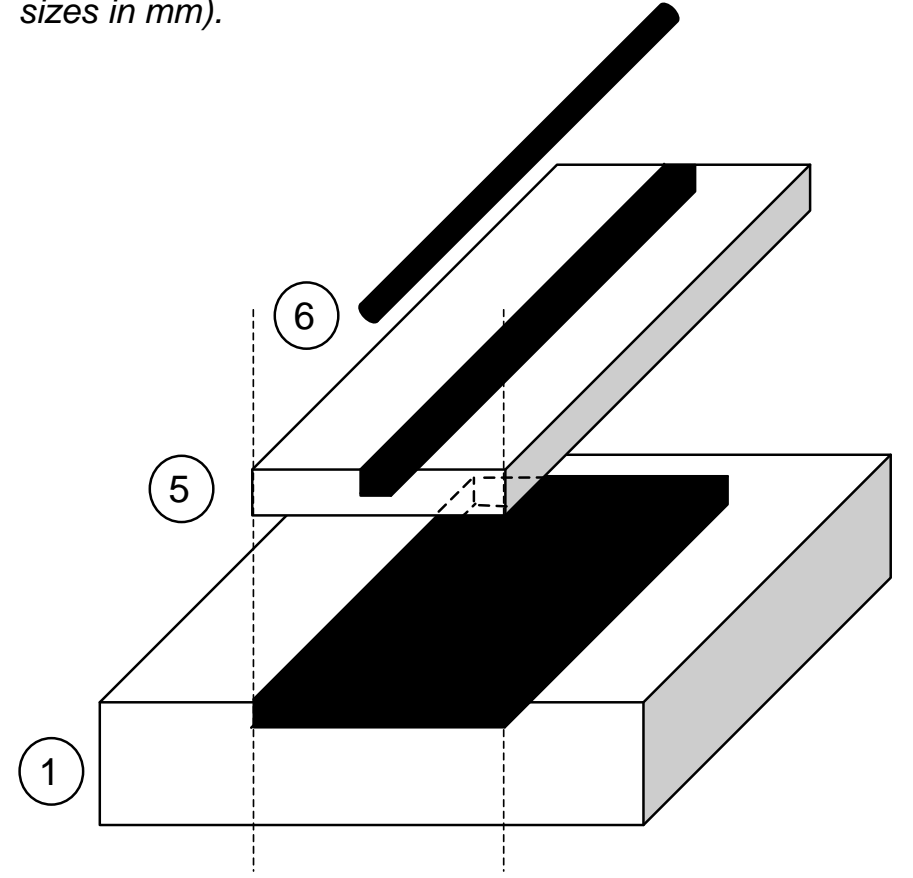

Figure A10 3-dimensional view of the film phantom. Part 1 and part 5 correspond to the parts in Figure A9 and part 6 is the source (in case of the Guidant source) or the physics catheter (in case of the Novoste setup). 


\section{Appendix 7. Technical drawings of phantoms for ophthalmic sources}

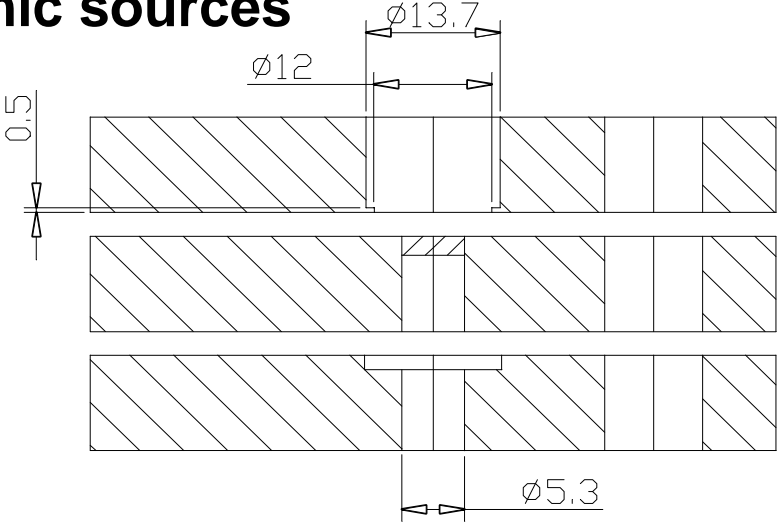

Figure A11 Phantom for plastic scintillator measurements on planar SIA.20 source. Solid water spacers can be inserted for measurements at different depths. The upper part of the phantom includes a small step through which the source can be centered (all sizes in $\mathrm{mm}$ ).

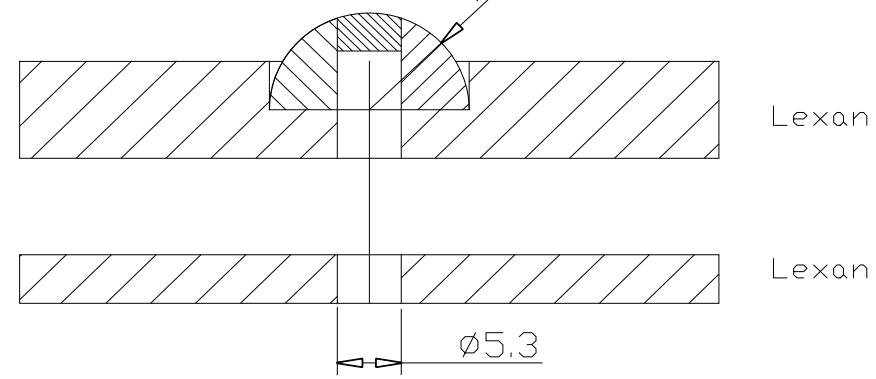

Figure A12 Phantom for plastic scintillator measurements on concave strontium (radius of curvature $\left.R_{c}=10 \mathrm{~mm}\right)$ or ruthenium $\left(R_{c}=12 \mathrm{~mm}\right)$ sources. Solid water spacers can be inserted for measurements at different depths. These spacers are convex on the top (all sizes in $\mathrm{mm}$ ).

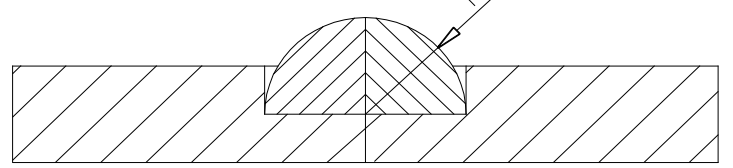

Figure $A 13$ Phantom for radiochromic film measurements on concave strontium $\left(R_{c}=10 \mathrm{~mm}\right)$ or ruthenium $\left(R_{c}=12 \mathrm{~mm}\right)$ sources. The film can be sandwiched between the two half spheres. $A$ spacing of $0.2 \mathrm{~mm}$ is kept to compensate for film thickness.

Table A12 Phantom spacer sizes (in $\mathrm{mm}$ ) for all solid water (RMI-457) phantoms. Measurements were corrected for small differences in depth according to fitting polynomials. The measurement depth includes the size and position of the detection volume.

\begin{tabular}{|c|c|c|c|c|}
\hline $\begin{array}{c}\text { Spacer } \\
(\#)\end{array}$ & $\begin{array}{c}\text { Phantom SIA.20 } \\
\text { (strontium) }\end{array}$ & $\begin{array}{c}\text { Phantom SIA.6 } \\
\text { (strontium) }\end{array}$ & $\begin{array}{c}\text { Phantom CCi } \\
\text { (ruthenium) }\end{array}$ & $\begin{array}{c}\text { (corrected) } \\
\text { measurement depth }\end{array}$ \\
\hline 1 & 1.15 & 1.12 & 1.08 & 2 \\
\hline 2 & 2.15 & 2.12 & 2.08 & 3 \\
\hline 3 & 3.15 & 3.12 & 3.08 & 4 \\
\hline 4 & 4.15 & 4.12 & 4.08 & 5 \\
\hline 5 & 9.15 & 9.12 & 9.08 & 10 \\
\hline
\end{tabular}




\section{Appendix 8. Setup for measurements on ophthalmic sources}

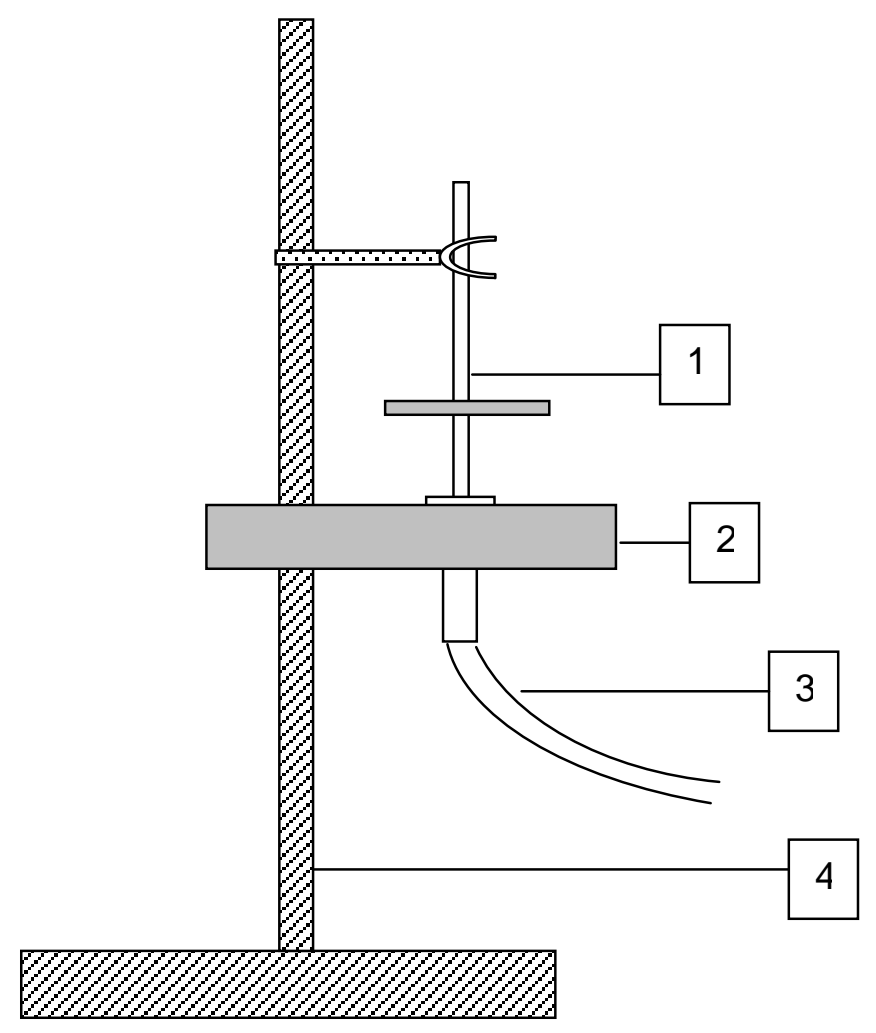

Figure A14 Measurement setup for measurements on ophthalmic sources. 1: ophthalmic applicator, 2: phantom, 3: detector (plastic scintillator or plane-parallel ionization chamber), 4: tripod. The source is facing downwards. The setup is such that the source is centered with respect to the detector. 


\section{Appendix 9. Setup for in-water diode measurements on ruthenium sources}

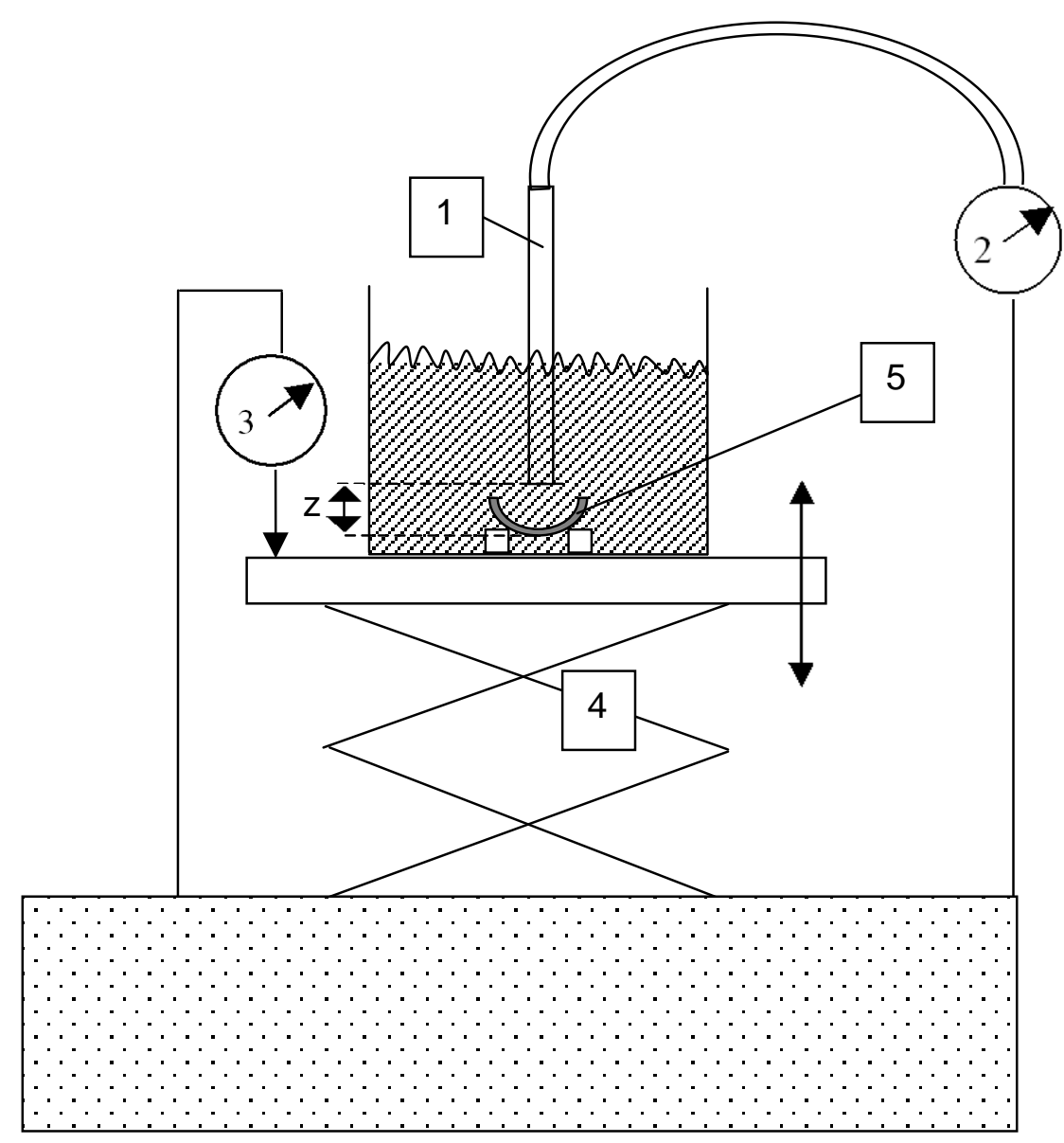

Figure A15 Measurement setup for in-water measurements on ruthenium sources. 1: diode, 2: electrometer, 3: depth dial indicator, 4: translation stage (only in z-direction), 5: ruthenium source in water. Depth dial indicator and diode were fixed in position (with respect to the ground), the source could be moved up and down through the translation stage to create different source-detector distances ( $z$ ). 


\section{Appendix 10. Measurement procedures}

\section{Appendix 10.1 Measurement procedure Guidant ${ }^{32} \mathrm{P}$}

\section{Well-type ionization chamber}

Positioning of source

- $\quad$ Measure at maximum of sweet spot (after determination of sweet spot by stepping the source)

- $\quad$ Straight physics coupler

Positioning of detector

- $\quad$ Behind (perspex) protection screen

- Influences of backscatter are negligible for measurements on beta sources

Number of measurements; achievable statistics

- $\quad \mathrm{SD}<0.1 \%$ can be achieved with $\mathrm{N}=6$ integrating measurements $(60 \mathrm{~s})$. Use repeated source insertions.

Detector stability

- $\quad$ Check correct voltage and polarity (according to calibration, for example -300 V)

- $\quad$ Take 30 minutes warm-up time into account (with chamber connected)

- $\quad$ Zero electrometer; correct for temperature and pressure

\section{Plastic scintillator}

Positioning of source

For the phantom used, the middle of the source should be at position 1982 as defined in the afterloader.

Positioning of detector

- $\quad$ Behind (perspex) protection screen

- $\quad$ At $2 \mathrm{~mm}$. No air gaps!

- Keep probe in same shape during different measurements (signal depends on shape of probe).

Number of measurements; achievable statistics

- $\quad \mathrm{SD}<1 \%$ should be achievable with $N=5$ integrating measurements $(60 \mathrm{~s})$. Use repeated source insertions.

For determination of $\dot{D}\left(r_{0}\right)$ use integrations with the source at at least 3 positions taking into account a margin of at least $2.5 \mathrm{~mm}$ from the $50 \%$ point.

Detector stability

- $\quad$ Take into account 30 minutes warm-up time (with scintillator probe connected)

- Zero detector

- $\quad$ Perform check source measurements every 40 minutes (range low). Changes higher than 2\% should not be tolerated. The change can be concluded from the different calibration factors after a check source measurement.

Radiochromic film

Positioning of detector

- $\quad$ Behind (perspex) protection screen

- $\quad$ Measurements at $2 \mathrm{~mm}$ waterequivalent

- $\quad$ Careful with air gaps in phantom

Film handling

- $\quad$ Mark proximal and distal side of the source on the film

- Cut film in direction of coating

- Keep films out of light as much as possible and prevent high temperatures

- $\quad$ Calibrate film and take same time between irradiation and densitometry into account

- $\quad$ Preferably use HD810 film

Film scanning (some points specific for flatbed scanner) In transmission mode

- At for example 300 dpi or higher

- $\quad$ At $>8$ bits TIF format (if possible)

- Do not allow scan (color) corrections

- $\quad$ No direct contact between scanner plate and film (Newton rings will appear)

- $\quad$ Scan direction parallel to the film (no angle) 


\section{Appendix 10.2 Measurement procedure Novoste ${ }^{90} \mathrm{Sr} /{ }^{90} \mathrm{Y}$}

Well-type ionization chamber

Positioning of source

- $\quad$ Measurement in sweet spot (IVB1000 is equipped with a 30, 40 and $60 \mathrm{~mm}$ source insert position)

- $\quad$ Averaging over 4 compass directions to eliminate directional dependence

Positioning of detector

- $\quad$ Behind (perspex) protection screen

- Influences of backscatter are negligible for measurements on beta sources

- Clinical catheter should be fixed to chamber insert

Number of measurements; achievable statistics

- $\quad \mathrm{SD}<1 \%$ can be achieved with $\mathrm{N}=3 \times 4$ integrating measurements $(60 \mathrm{~s})$. Use multiple positioning of source and catheter (for example 3 repeated source insertions and 4 compass directions turning both insert and catheter together).

Detector stability

- $\quad$ Check correct voltage and polarity (according to calibration, for example -300 V)

- Take 30 minutes warm-up time into account (with chamber connected)

- Zero electrometer; correct for temperature and pressure

Warning: our experience was that in measurements with the clinical 3.5F catheter (summer 2002) the source could get stuck in the clinical catheter when the catheter was used more than once.

\section{Plastic scintillator}

Positioning of source

- Stable source positioning should be monitored. Keeping pressure on water in the afterloader with a rubber band, positioning the phantom in an angle are possibilities to keep the seed train in a stable position

Positioning of detector

- $\quad$ Behind (perspex) protection screen

- $\quad$ At $2 \mathrm{~mm}$. No air gaps! Keep probe in same shape during different measurements (signal depends on shape of probe).

Number of measurements; achievable statistics

- $\quad S d<3 \%$ should be achievable with $N=5$ integrating measurements $(60 \mathrm{~s})$. Use repeated source insertions. For determination of $\dot{D}\left(r_{0}\right)$ use integrations with the source at at least 3 positions taking into account a margin of at least $3.0 \mathrm{~mm}$ from the $50 \%$ point.

Detector stability

- Take into account 30 minutes warm-up time (with scintillator probe connected)

- Zero detector

- Perform check source measurements every 40 minutes (range low). Changes higher than 2\% should not be tolerated. The change can be concluded from the different calibration factors after a check source measurement.

Radiochromic film

Positioning of source

- Keep pressure on water in the afterloader, for example with a rubber band, position the phantom in an angle to keep the seed train in a stable position.

Positioning of detector

- $\quad$ Behind (perspex) protection screen

- $\quad$ Measurements at $2 \mathrm{~mm}$ waterequivalent

- $\quad$ Careful with air gaps in phantom

Film handling

- Mark proximal and distal side of the source on the film

- Cut film in direction of coating

- Keep films out of light as much as possible and prevent high temperatures

- Calibrate film and take same time between irradiation and densitometry into account

- $\quad$ Preferably use HD810 film

Film scanning (some points specific for flatbed scanner) In transmission mode

- At for example 300 dpi or higher

- $\quad$ At $>8$ bits TIF format (if possible)

- $\quad$ Do not allow scan (color) corrections

- No direct contact between scanner plate and film (Newton rings will appear)

- Scan direction parallel to the film (no angle) 


\section{Appendix 10.3 Measurement procedure Amersham ${ }^{90} \mathrm{Sr} /{ }^{90} \mathrm{Y}$}

\section{Plastic scintillator}

Positioning of source

- $\quad$ Place source horizontally

- $\quad$ Source centered with respect to detector (axis coinciding with axis of detector)

Positioning of detector

- $\quad$ Behind (perspex) protection screen

- $\quad$ Avoid air gaps! Keep probe in same shape during different measurements (signal depends on shape of probe).

Number of measurements; achievable statistics

- $\quad \mathrm{SD}<2 \%$ should be achievable with $\mathrm{N}=5$ measurements, $\mathrm{SD}<1 \%$ for concave sources. Use repeated source placements.

Detector stability

- Take into account 30 minutes warm-up time (with scintillator probe connected)

- Zero detector

- $\quad$ Perform check source measurements every 40 minutes (range low). Changes higher than 2\% should not be tolerated. The change can be concluded from the different calibration factors after a check source measurement.

Plane-parallel ionization chamber

Positioning of source

- $\quad$ Place source horizontally

- $\quad$ Source centered with respect to detector (axis coinciding with axis of detector)

Positioning of detector

- $\quad$ Behind (perspex) protection screen

- $\quad$ Avoid air gaps!

- The position of the detector should be fixed, especially when it is positioned under the source

- The detector should be properly centered with respect to the phantom

Number of measurements; achievable statistics

- $\quad \mathrm{SD}<1 \%$ should be achievable with $\mathrm{N}=5$ measurements

Detector stability

- $\quad$ Check correct voltage and polarity (according to calibration, for example -300 V)

- $\quad$ Take into account 30 minutes warm-up time (with chamber connected)

- $\quad$ Zero electrometer; correct for temperature and pressure

- $\quad$ As a verification of the stability of the chamber check source measurements can be performed. No differences larger than $1 \%$ were observed

Radiochromic film

Positioning of source

- Place source horizontally

- $\quad$ Beware of source movements

Positioning of detector

- Behind (perspex) protection screen

- $\quad$ Careful with air gaps in phantom

- Use waterequivalent backscatter material

Film handling

- Mark proximal and distal side of the source on the film

- $\quad$ Keep films out of light as much as possible and prevent high temperatures

- $\quad$ Calibrate film and take same time between irradiation and densitometry into account

- $\quad$ Preferably use HD810 film if the dose rate allows it

Film scanning (some points specific for flatbed scanner)

- In transmission mode

- $\quad$ At for example 300 dpi or higher

- $\quad$ At $>8$ bits TIF format (if possible)

- Do not allow scan (color) corrections

- $\quad$ No direct contact between scanner plate and film (Newton rings will appear)

- Scan direction parallel to the film (no angle) 


\section{Appendix 10.4 Measurement procedure BEBIG ${ }^{106} \mathrm{Ru} /{ }^{106} \mathrm{Rh}$}

\section{Plastic scintillator}

Positioning of source

- Place source horizontally

- Source centered with respect to detector (axis coinciding with axis plastic scintillator).

Positioning of detector

- Behind (perspex) protection screen

- Avoid air gaps! Keep probe in same shape during different measurements (signal depends on shape of probe).

Number of measurements; achievable statistics

- $\quad S D<1 \%$ should be achievable with $N=5$ measurements (in phantom, sources without cutouts). Use repeated source placements

Detector stability

- Take into account 30 minutes warm-up time (with scintillator probe connected)

- Zero detector

- Perform check source measurements every 40 minutes (range low). Changes higher than $\sim 2 \%$ should not be tolerated. The change can be concluded from the different calibration factors after a check source measurement.

\section{Diode}

Positioning of source

- Source centered with respect to detector (axis coinciding with axis plastic scintillator)

Positioning of detector

- Behind (perspex) protection screen

- In the case of in water measurements a depth dial indicator can be used for determination of the sourcedetector distance.

Detector stability

- $\quad$ No warm-up time required

- Check correct voltage $(0 \mathrm{~V})$

- Zero detector

- Our experience is that a diode detector can be applied without check source measurements, but only when it is not used for other measurements (no doses $>100 \mathrm{~Gy}$ )

Radiochromic film

Positioning of source

- Place source horizontally

- Beware of source movements

Positioning of detector

- Behind (perspex) protection screen

- Careful with air gaps in phantom

- Use waterequivalent backscatter material

Film handling

- Keep films out of light as much as possible and prevent high temperatures

- Calibrate film and take same time between irradiation and densitometry into account

- Use HS or MD55-2 film for uniformity measurements, MD55-2 film for depth dose measurements

Film scanning (some points specific for flatbed scanner)

- In transmission mode

- At for example 300 dpi or higher

- $\quad$ At $>8$ bits TIF format (if possible)

- Do not allow scan (color) corrections

- $\quad$ No direct contact between scanner plate and film (Newton rings will appear)

- Scan direction parallel to the film (no angle) 


\section{Appendix 11. Choice of margin source non-uniformity $U_{F}$}

The AAPM criterion - based on 2/3 of the source length - sets large margins from the ends of the source. On the basis of Monte Carlo data it may be concluded that smaller margins would be more realistic. A margin that is equally demanding for all beta sources would be better; therefore, here a distinction is made between margins for the phosphorus and strontium line sources. Figure A16 and Figure A17 represent the same HD810 film data as presented in Chapter 5. Different margins are applied to assess the effects of the choice of margin on the magnitude of the source non-uniformity $U_{F}$.

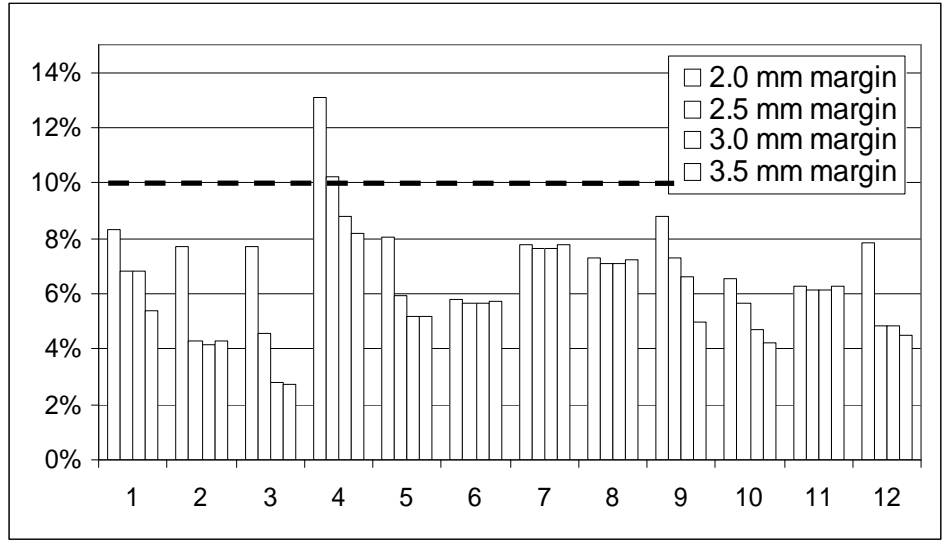

Figure A16 Guidant source non-uniformity $U_{F}$ (with HD810) for different margins:

2.0, 2.5, 3.0 and $3.5 \mathrm{~mm}$.

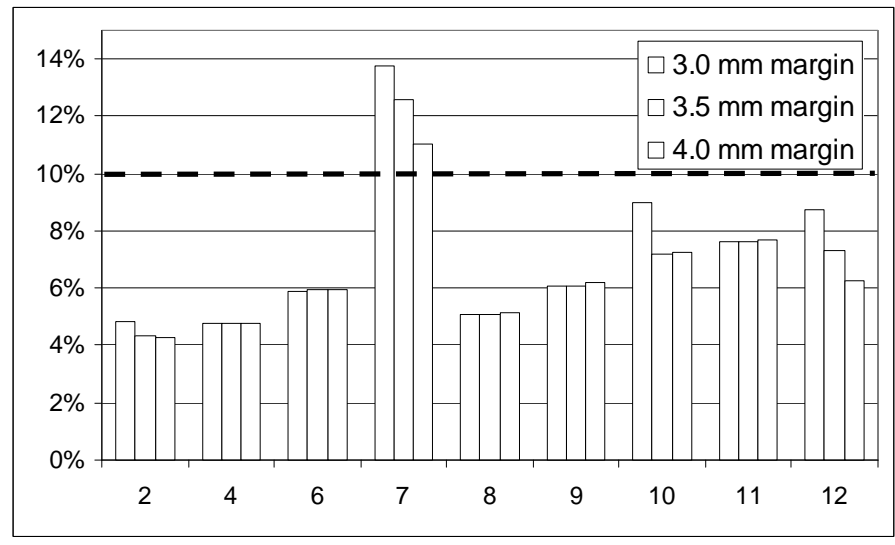

Figure A17 Novoste source non-uniformity $U_{F}$ (with HD810) for different margins:

3.0, 3.5 and $4.0 \mathrm{~mm}$.

The figures show that the magnitude of the non-uniformity is not strongly correlated with the size of the margin. However, non-uniform sources like Guidant source 4 and Novoste source 7 , do show a dependence on the choice of the margin, because the non-uniformity is close to the edge of the source. The Monte Carlo data from Kirisits et al [32] show that for the Guidant sources $\sim 97 \%$ of the dose maximum is reached with a margin of $2.5 \mathrm{~mm}$ and that for the Novoste sources $~ 96 \%$ is reached with a margin of $3.0 \mathrm{~mm}$ (Figure A18). It is, therefore, proposed to use a margin of $2.5 \mathrm{~mm}$ for the Guidant sources and $3.0 \mathrm{~mm}$ for the Novoste sources.

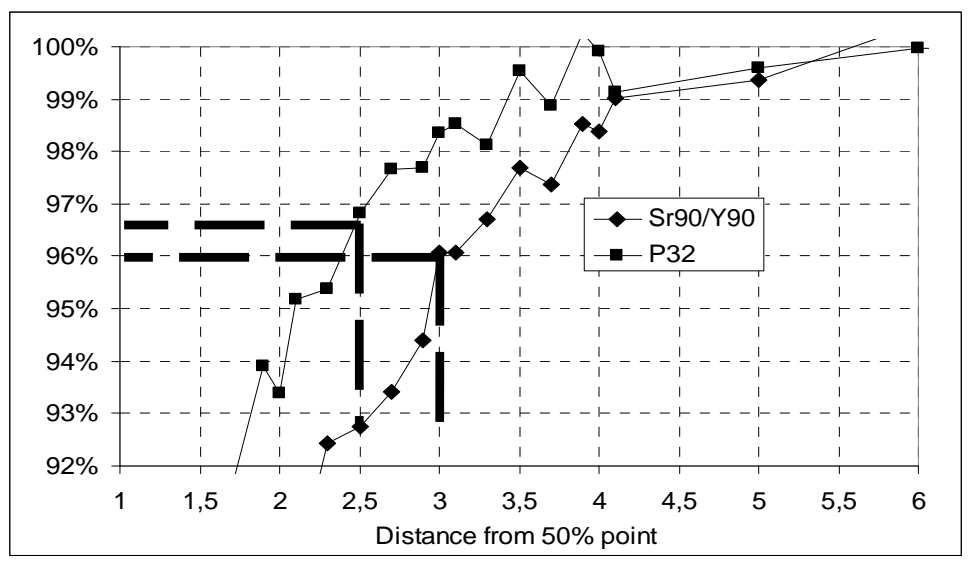

Figure A18 Source profiles for Guidant and Novoste sources. The choice for a margin of $2.5 \mathrm{~mm}$ (Guidant) and $3.0 \mathrm{~mm}$ (Novoste) is added to the graph. 


\section{Origin of figures}

The following list gives an overview of the figures taken from other sources. The figures were used with permission of the authors. No figures or text in this report may be reproduced without permission, in writing, from the authors.

Figure 1-1 Example of pterygium. 8 Rutnin Eye Hospital (Bangkok, Thailand), http://www.rutnin.com/html/c_common.html

Figure 1-2 Example of a choroidal melanoma University of Illinois at Chicago Medical Center, Dept. of Ophthalmology and Visual Sciences (Chicago, IL, USA) http://www.uic.edu/com/eye/education/eyefacts/ChoroidalMelanoma.htm

Figure 1-3 Example of in-stent restenosis, primary indication for intravascular brachytherapy.... 7 Novoste Corporation (Norcross, GA, USA)

Figure 1-4 The Guidant automatic afterloader. Guidant Vascular Intervention Inc. (Houston, TX, USA)

Figure 1-5 The Novoste handheld afterloader. 9 Novoste Corporation (Norcross, GA, USA)

Figure 2-1 Schematic view of the $5 \mathrm{~F}{ }^{90} \mathrm{Sr} /{ }^{90} \mathrm{Y}$ Novoste source 10 Novoste Corporation (Norcross, GA, USA)

Figure 2-2 Schematic view of the $20 \mathrm{~mm}{ }^{32} \mathrm{P}$ Guidant source. 10 Guidant Vascular Intervention Inc. (Houston, TX, USA)

Figure 2-3 Configuration of the planar SIA.20 strontium-90 source. Amersham Health (Buckinghamshire, UK)

Figure 2-4 Configuration of the concave SIA.6 strontium-90 source. Amersham Health (Buckinghamshire, UK)

Figure 2-5 Configuration of ruthenium-106 plaques. BEBIG Isotopen- und Medizintechnik GmbH (Berlin, Germany)

Figure 3-2 ASL as defined in the EVA GEC ESTRO document. 14 Edited from reference [8]

Figure $A 3$ Cross-section of the ruthenium plaque. 71 $B E B I G$ Isotopen- und Medizintechnik GmbH (Berlin, Germany)

Figure A4 Plane view of the sources described in Table A2. 71 BEBIG Isotopen- und Medizintechnik GmbH (Berlin, Germany)

Figure A6 PTW phantom for plastic scintillator measurements on Novoste ${ }^{90} \mathrm{Sr} /{ }^{90} \mathrm{Y}$ source trains W. Weber, Novoste GmbH (Krefeld, Germany) 


\section{References}

[1] Elfrink, R.J., Kolkman-Deurloo, I.K., van Kleffens, H.J., Rijnders, A., Schaeken, B., Aalbers, T.H., Dries, W.J. and Venselaar, J.L.

Quality control of brachytherapy equipment in the Netherlands and Belgium: current practice and minimum requirements

Radiother.Oncol. 62: 95-102, 2002

[2] ICRU (International Commission on Radiation Units and Measurements)

Dosimetry of beta rays and low-energy photons for brachytherapy with sealed sources ICRU Report 72, preliminary version, August 2001

[3] IAEA (International Atomic Energy Agency)

Calibration of photon and beta ray sources used in brachytherapy

IAEA-TECDOC-1274, Vienna, 2002

http://www-naweb.iaea.org/nahu/external/e3/publications.asp

[4] Nath, R., Amols, H., Coffey, C., Duggan, D., Jani, S., Li, Z., Schell, M., Soares, C., Whiting, J., Cole, P.E., Crocker, I. and Schwartz, R.

Intravascular brachytherapy physics: report of the AAPM Radiation Therapy Committee Task Group no. 60. American Association of Physicists in Medicine

Med.Phys. 26: 119-152, 1999

[5] DGMP (Deutsche Gesellschaft fur Medizinische Physik e.V.)

Guidelines on Medical Physical Aspects of Intravascular Brachytherapy.

Report 16, 2001

http://www.dgmp.de/Page_Papiere/Report16.pdf

[6] Nath, R., Anderson, L.L., Meli, J.A., Olch, A.J., Stitt, J.A. and Williamson, J.F. Code of practice for brachytherapy physics: report of the AAPM Radiation Therapy Committee Task Group No. 56. American Association of Physicists in Medicine Med.Phys. 24: 1557-1598, 1997

[7] NCS (Netherlands Commission on Radiation Dosimetry)

Quality Control in Brachytherapy. Current practice and minimum requirements.

Report 13, 2000

[8] Potter, R., Van Limbergen, E., Dries, W., Popowski, Y., Coen, V., Fellner, C., Georg, D., Kirisits, C., Levendag, P., Marijnissen, H., Marsiglia, H., Mazeron, J.J., Pokrajac, B., Scalliet, P. and Tamburini, V.

Prescribing, recording, and reporting in endovascular brachytherapy. Quality assurance, equipment, personnel and education

Radiother.Oncol. 59: 339-360, 2001

[9] Holmes, D.R., Jr., Vlietstra, R.E., Smith, H.C., Vetrovec, G.W., Kent, K.M., Cowley, M.J., Faxon, D.P., Gruentzig, A.R., Kelsey, S.F. and Detre, K.M.

Restenosis after percutaneous transluminal coronary angioplasty (PTCA): a report from the PTCA Registry of the National Heart, Lung, and Blood Institute Am.J.Cardiol. 53: 77C-81C, 1984 
[10] Califf, R.M., Fortin, D.F., Frid, D.J., Harlan, W.R., III, Ohman, E.M., Bengtson, J.R., Nelson, C.L., Tcheng, J.E., Mark, D.B. and Stack, R.S.

Restenosis after coronary angioplasty: an overview J.Am.Coll.Cardiol. 17: 2B-13B, 1991

[11] Serruys, P.W., de Jaegere, P., Kiemeneij, F., Macaya, C., Rutsch, W., Heyndrickx, G., Emanuelsson, H., Marco, J., Legrand, V. and Materne, P.

A comparison of balloon-expandable-stent implantation with balloon angioplasty in patients with coronary artery disease. Benestent Study Group

N.Engl.J.Med. 331: 489-495, 1994

[12] Popowski, Y., Verin, V. and Urban, P.

Endovascular beta-irradiation after percutaneous transluminal coronary balloon angioplasty

Int.J.Radiat.Oncol.Biol.Phys. 36: 841-845, 1996

[13] Waksman, R., Cheneau, E., Ajani, A.E., White, R.L., Pinnow, E., Torguson, R., Deible, R., Satler, L.F., Pichard, A.D., Kent, K.M., Teirstein, P.S. and Lindsay, J.

Intracoronary radiation therapy improves the clinical and angiographic outcomes of diffuse in-stent restenotic lesions: results of the Washington Radiation for In-Stent Restenosis Trial for Long Lesions (Long WRIST) Studies

Circulation 107: 1744-1749, 2003

[14] Waksman, R., Raizner, A.E., Yeung, A.C., Lansky, A.J. and Vandertie, L. Use of localised intracoronary beta radiation in treatment of in-stent restenosis: the INHIBIT randomised controlled trial Lancet 359: 551-557, 2002

[15] Urban, P., Serruys, P., Baumgart, D., Colombo, A., Silber, S., Eeckhout, E., Gershlick, A., Wegscheider, K., Verhees, L. and Bonan, R.

A multicentre European registry of intraluminal coronary beta brachytherapy

Eur.Heart J. 24: 604-612, 2003

[16] Verin, V., Popowski, Y., de Bruyne, B., Baumgart, D., Sauerwein, W., Lins, M., Kovacs, G., Thomas, M., Calman, F., Disco, C., Serruys, P.W. and Wijns, W.

Endoluminal beta-radiation therapy for the prevention of coronary restenosis after balloon angioplasty. The Dose-Finding Study Group

N.Engl.J.Med. 344: 243-249, 2001

[17] Raizner, A.E., Oesterle, S.N., Waksman, R., Serruys, P.W., Colombo, A., Lim, Y.L., Yeung, A.C., van der Giessen, W.J., Vandertie, L., Chiu, J.K., White, L.R., Fitzgerald, P.J., Kaluza, G.L. and Ali, N.M.

Inhibition of restenosis with beta-emitting radiotherapy: Report of the Proliferation Reduction with Vascular Energy Trial (PREVENT)

Circulation 102: 951-958, 2000

[18] King, S.B., III, Williams, D.O., Chougule, P., Klein, J.L., Waksman, R., Hilstead, R., Macdonald, J., Anderberg, K. and Crocker, I.R.

Endovascular beta-radiation to reduce restenosis after coronary balloon angioplasty: results of the beta energy restenosis trial (BERT)

Circulation 97: 2025-2030, 1998 
[19] Teirstein, P.S., Massullo, V., Jani, S., Popma, J.J., Mintz, G.S., Russo, R.J., Schatz, R.A., Guarneri, E.M., Steuterman, S., Morris, N.B., Leon, M.B. and Tripuraneni, P. Catheter-based radiotherapy to inhibit restenosis after coronary stenting N.Engl.J.Med. 336: 1697-1703, 1997

[20] Leon, M.B., Teirstein, P.S., Moses, J.W., Tripuraneni, P., Lansky, A.J., Jani, S., Wong, S.C., Fish, D., Ellis, S., Holmes, D.R., Kerieakes, D. and Kuntz, R.E. Localized intracoronary gamma-radiation therapy to inhibit the recurrence of restenosis after stenting

N.Engl.J.Med. 344: 250-256, 2001

[21] Franken, Y. and Huyskens, C.J.

Balancing the use of radiation in endovascular brachytherapy

Proceedings of SRP International Symposium in Southport, 1999

[22] Hiatt, B.L., Ikeno, F., Yeung, A.C. and Carter, A.J.

Drug-eluting stents for the prevention of restenosis: In quest for the holy grail

Catheter.Cardiovasc.Interv. 55: 409-417, 2002

[23] Regar, E., Sianos, G. and Serruys, P.W.

Stent development and local drug delivery

Br.Med.Bull. 59: 227-248, 2001

[24] Sinclair, W.K. and Trott, N.G.

The construction and measurement of beta-ray applicators for use in ophthalmology Br.J.Radiol. 29: 15-23, 1956

[25] Fukushima, S., Inoue, T., Inoue, T. and Ozeki, S.

Postoperative irradiation of pterygium with ${ }^{90} \mathrm{Sr}$ eye applicator

Int.J.Radiat.Oncol.Biol.Phys. 43: 597-600, 1999

[26] van den Brenk, H.A.S.

Results of prophylactic postoperative irradiation in 1300 cases of pterygium

Am.J.Roentgenol. 103: 723-733, 1968

[27] Seregard, S.

Long-term survival after ruthenium plaque radiotherapy for uveal melanoma. A metaanalysis of studies including 1,066 patients

Acta Ophthalmol.Scand. 77: 414-417, 1999

[28] Hernandez, J.C., Brady, L.W., Shields, C.L., Shields, J.A. and DePotter, P. Conservative treatment of retinoblastoma. The use of plaque brachytherapy Am.J.Clin.Oncol. 16: 397-401, 1993

[29] Lax, I.

Dosimetry of ${ }^{106} \mathrm{Ru}$ eye applicators with a p-type silicon detector

Phys.Med.Biol. 36: 963-972, 1991

[30] Missotten, L., Dirven, W., Van der Schueren, A., Leys, A., De Meester, G. and Van Limbergen, E.

Results of treatment of choroidal malignant melanoma with high-dose- rate strontium90 brachytherapy. A retrospective study of 46 patients treated between 1983 and 1995 Graefes Arch.Clin.Exp.Ophthalmol. 236: 164-173, 1998 
[31] Lommatzsch, P.K., Werschnik, C. and Schuster, E.

Long-term follow-up of Ru-106/Rh-106 brachytherapy for posterior uveal melanoma Graefes Arch.Clin.Exp.Ophthalmol. 238: 129-137, 2000

[32] Kirisits, C., Georg, D., Wexberg, P., Pokrajac, B., Glogar, D. and Potter, R.

Determination and application of the reference isodose length (RIL) for commercial endovascular brachytherapy devices

Radiother.Oncol. 64: 309-315, 2002

[33] Soares, C.G. and McLaughlin, W.L.

Measurement of radial dose distributions around small beta particle emitters using high resolution radiochromic foil dosimetry

Rad.Prot.Dosim. 47: 367-372, 1993

[34] Soares, C.G., Vynckier, S., Jarvinen, H., Cross, W.G., Sipila, P., Fluhs, D., Schaeken, B., Mourtada, F.A., Bass, G.A. and Williams, T.T.

Dosimetry of beta-ray ophthalmic applicators: comparison of different measurement methods

Med.Phys. 28: 1373-1384, 2001

[35] Coursey, B.M., Colle, R., Zimmerman, B.E., Cessna, J.T. and Golas, D.B.

National radioactivity standards for beta-emitting radionuclides used in intravascular brachytherapy

Int.J.Radiat.Oncol.Biol.Phys. 41: 207-216, 1998

[36] Colle, R., Zimmerman, B.E., Soares, C.G. and Coursey, B.M.

Determination of a calibration factor for the nondestructive assay of Guidant ${ }^{32} \mathrm{P}$ brachytherapy sources

Appl.Radiat.Isot. 50: 835-841, 1999

[37] Goetsch, S.J., Attix, F.H., Pearson, D.W. and Thomadsen, B.R.

Calibration of ${ }^{192} \mathrm{Ir}$ high-dose-rate afterloading systems

Med.Phys. 18: 462-467, 1991

[38] Lambert, M.S., Micka, J.A., and DeWerd, L.A.

Calibration of well-type chambers for Novoste ${ }^{90} \mathrm{Sr}^{90} \mathrm{Y}$ intravascular brachytherapy source trains

Works in progress poster, AAPM annual meeting, Salt Lake City, 2001

[39] Ali, M.M. and Khan, F.M.

Determination of surface dose rate from a ${ }^{90} \mathrm{Sr}$ ophthalmic applicator

Med.Phys. 17: 416-421, 1990

[40] Reft, C.S., Kuchnir, F.T., Rosenberg, I. and Myrianthopoulos, L.C.

Dosimetry of Sr-90 ophthalmic applicators

Med.Phys. 17: 641-646, 1990

[41] Piessens, M. and Reynaert, N.

Verification of absolute dose rates for intravascular brachytherapy beta sources

Phys.Med.Biol. 45: 2219-2231, 2000 
[42] Niroomand-Rad, A., Blackwell, C.R., Coursey, B.M., Gall, K.P., Galvin, J.M., McLaughlin, W.L., Meigooni, A.S., Nath, R., Rodgers, J.E. and Soares, C.G. Radiochromic film dosimetry: recommendations of AAPM Radiation Therapy Committee Task Group 55. American Association of Physicists in Medicine Med.Phys. 25: 2093-2115, 1998

[43] Meigooni, A.S., Sanders, M.F., Ibbott, G.S. and Szeglin, S.R. Dosimetric characteristics of an improved radiochromic film Med.Phys. 23: 1883-1888, 1996

[44] Odero, D.O., Gluckman, G.R., Welsh, K., Wlodarczyk, R.A. and Reinstein, L.E. The use of an inexpensive red acetate filter to improve the sensitivity of GAFChromic dosimetry

Med.Phys. 28: 1446-1448, 2001

[45] Sayeg, J.A. and Gregory, R.C.

A new method for characterizing beta-ray ophthalmic applicator sources Med.Phys. 18: 453-461, 1991

[46] Soares, C.G.

A method for the calibration of concave ${ }^{90} \mathrm{Sr}+{ }^{90} \mathrm{Y}$ ophthalmic applicators Phys.Med.Biol. 37: 1005-1007, 1992

[47] Soares, C.G., Halpern, D.G. and Wang, C.K. Calibration and characterization of beta-particle sources for intravascular brachytherapy Med.Phys. 25: 339-346, 1998

[48] Taccini, G., Cavagnetto, F., Coscia, G., Garelli, S. and Pilot, A. The determination of dose characteristics of ruthenium ophthalmic applicators using radiochromic film Med.Phys. 24: 2034-2037, 1997

[49] Mourtada, F.A., Soares, C.G., Seltzer, S.M. and Lott, S.H. Dosimetry characterization of ${ }^{32} \mathrm{P}$ catheter-based vascular brachytherapy source wire Med.Phys. 27: 1770-1776, 2000

[50] Duggan, D.M., Coffey, C.W., Lobdell, J.L. and Schell, M.C. Radiochromic film dosimetry of a high dose rate beta source for intravascular brachytherapy Med.Phys. 26: 2461-2464, 1999

[51] Gluckman, G.R. and Reinstein, L.E. Comparison of three high-resolution digitizers for radiochromic film dosimetry Med.Phys. 29: 1839-1846, 2002

[52] Hafeli, U.O., Roberts, W.K., Meier, D.S., Ciezki, J.P., Pauer, G.J., Lee, E.J. and Weinhous, M.S.

Dosimetry of a W-188/Re-188 beta line source for endovascular brachytherapy Med.Phys. 27: 668-675, 2000 
[53] Chan, M.F., Fung, A.Y., Hu, Y.C., Chui, C.S., Amols, H., Zaider, M. and Abramson, D. The measurement of three dimensional dose distribution of a ruthenium- 106 ophthalmological applicator using magnetic resonance imaging of BANG polymer gels J.Appl.Clin.Med.Phys. 2: 85-89, 2001

[54] Maryanski, M.J., Ibbott, G.S., Eastman, P., Schulz, R.J. and Gore, J.C. Radiation therapy dosimetry using magnetic resonance imaging of polymer gels Med.Phys. 23: 699-705, 1996

[55] Meeks, S.L., Bova, F.J., Maryanski, M.J., Kendrick, L.A., Ranade, M.K., Buatti, J.M. and Friedman, W.A.

Image registration of BANG gel dose maps for quantitative dosimetry verification Int.J.Radiat.Oncol.Biol.Phys. 43: 1135-1141, 1999

[56] Schaart, D.R., Bos, A.J., Winkelman, A.J. and Clarijs, M.C.

The radial depth-dose distribution of $a^{188} \mathrm{~W} /{ }^{188} \mathrm{Re}$ beta line source measured with novel, ultra-thin TLDs in a PMMA phantom: comparison with Monte Carlo simulations Phys.Med.Biol. 47: 3605-3627, 2002

[57] Binder, W., Chiari, A. and Aiginger, $\mathrm{H}$.

Determination of the dose distribution of an ophthalmic ${ }^{106} \mathrm{Ru}$ irradiator with TLDs and an eye phantom

Rad.Prot.Dosim. 34: 275-278, 1990

[58] Siddle, D. and Langmack, K.

Calibration of strontium-90 eye applicator using a strontium external beam standard Phys.Med.Biol. 44: 1597-1608, 1999

[59] Fluhs, D., Heintz, M., Indenkampen, F. and Wieczorek, C.

Direct reading measurement of absorbed dose with plastic scintillators-- the general concept and applications to ophthalmic plaque dosimetry

Med.Phys. 23: 427-304, 1996

[60] Bambynek, M., Fluhs, D., Quast, U., Wegener, D. and Soares, C.G.

A high-precision, high-resolution and fast dosimetry system for beta sources applied in cardiovascular brachytherapy

Med.Phys. 27: 662-667, 2000

[61] Piessens, M., Reynaert, N., Potempa, J., Thierens, H., Wijns, W. and Verbeke, L. Dose distributions for ${ }^{90} \mathrm{Y}$ intravascular brachytherapy sources used with balloon catheters

Med.Phys. 29: 1562-1571, 2002

[62] Rosenthal, P., Weber, W., Forster, A., Orth, O., Kohler, B. and Seiler, F. Calibration and validation of a quality assurance system for $90 \mathrm{Sr} / 90 \mathrm{Y}$ radiation source trains

Phys.Med.Biol. 48: 573-585, 2003

[63] Heydarian, M., Hoban, P.W., Beckham, W.A., Borchardt, I.M. and Beddoe, A.H. Evaluation of a PTW diamond detector for electron beam measurements Phys.Med.Biol. 38: 1035-1042, 1993 
[64] Vatnitsky, S. and Jarvinen, $\mathrm{H}$.

Application of a natural diamond detector for the measurement of relative dose distributions in radiotherapy

Phys.Med.Biol. 38: 173-184, 1993

[65] ICRU (International Commission on Radiation Units and Measurements) Dosimetry of External Beta Rays for Radiation Protection ICRU Report 56, Bethesda, MD, USA, 1997

[66] Rikner, G. and Grusell, E.

General specifications for silicon semiconductors for use in radiation dosimetry Phys.Med.Biol. 32: 1109-1117, 1987

[67] Davelaar, J., Schaling, D.F., Hennen, L.A. and Broerse, J.J. Dosimetry of ruthenium-106 eye applicators Med.Phys. 19: 691-694, 1992

[68] Schaeken, B. and Scalliet, P. One year of experience with alanine dosimetry in radiotherapy Appl.Radiat.Isot. 47: 1177-1182, 1996

[69] Pruit, J.S., Soares, C.G., and Ehrlich, M. Calibration of beta-particle radiation instrumentation and sources. NBS Special publication 250-21, 1988

[70] Soares, C.G.

Calibration of ophthalmic applicators at NIST: a revised approach Med.Phys. 18: 787-793, 1991

[71] Cross, W.G.

Variation of beta dose attenuation in different media Phys.Med.Biol. 13: 611-618, 1968

[72] Schaart, D.R. The scaling method applied to beta particle line sources with a finite diameter Med.Phys. 29: 2682-2686, 2002

[73] Hall, E.J., Miller, R.C. and Brenner, D.J. Radiobiological principles in intravascular irradiation Cardiovascular Radiation Medicine 1: 42-47, 1999

[74] Beyer, D.C.

Pterygia: single-fraction postoperative beta irradiation Radiology 178: 569-571, 1991

[75] Reinstein, L.E., Gluckman, G.R., Pai, S., and Meek, A. Post-irradiation dependence of radiochromic film optical density on temperature and time Med.Phys. 24: 1005, 1997

[76] Zhu, Y., Kirov, A.S., Mishra, V., Meigooni, A.S. and Williamson, J.F. Quantitative evaluation of radiochromic film response for two- dimensional dosimetry Med.Phys. 24: 223-231, 1997 
[77] Firestone, R.B.

Table of Isotopes

Eighth Edition, New York, 1996

[78] Kocher, D.C.

Radioactive decay data tables

Report DOE/TIC-11026, U.S. Department of Energy, Washington, D.C., 1981

[79] IAEA (International Atomic Energy Agency)

Absorbed dose determination in external beam radiotherapy - An international code of practice for dosimetry based on standards of absorbed dose to water -

Technical Reports series no. 398, Vienna, 2000 


\section{Publications of the Netherlands Commission on Radiation Dosimetry}

Radiation dosimetry activities in the Netherlands.

Inventory compiled under the auspices of the Netherlands Commission for Radiation

n.a.

Dosimetry.

NCS Report 1, July 1986.

Code of practice for the dosimetry of high-energy photon beams.

n.a.

NCS Report 2, December 1986.

Proceedings of the Symposium on Thermoluminescence Dosimetry.

n.a.

NCS Report 3, October 1988.

Aanbevelingen voor dosimetrie en kwaliteitscontrole van radioactieve bronnen bij

n.a.

brachytherapie

NCS Report 4, Februari 1989 (in Dutch).

Recommendations for dosimetry and quality control of radioactive sources used in

n.a.

brachytherapy.

Synopsis (in English) of NCS Report 4, February 1991.

Code of practice for the dosimetry of high-energy electron beams.

n.a.

NCS Report 5, December 1989.

Dosimetric aspects of Mammography.

n.a

NCS Report 6, March 1993.

Recommendations for the calibration of Iridium-192 high dose rate sources.

NCS Report 7, December 1994.

Kwaliteitscontrole van Medische Lineaire Versnellers, methoden voor kwaliteitscontrole, $€ 12,50$ wenselijke toleranties en frequenties.

NCS Report 8, December 1995 (in Dutch).

Quality Control of Medical Linear Accelerators, current practice and minimum requirements. $€ 12,50$ NCS Report 9, August 1996.

Dosimetry of low and medium energy $x$-rays, a code of practice for use in radiotherapy and $€ 12,50$ radiobiology.

NCS Report 10, July 1997.

Quality Control of Simulators and CT scanners and some basic QC methods for Treatment $€ 12,50$ Planning Systems, current practice and minimum requirements.

NCS Report 11, September 1997.

Determination and use of scatter correction factors of megavoltage photon beams,

measurement and use of collimator and phantom scatter correction factors of arbitrarily shaped fields with a symmetrical collimator setting.

NCS Report 12, March 1998.

NCS Report 13, October 2000. 
From outside The Netherlands, please complete the order form below.

\section{ORDER FORM}

Please send this completed order form to:

NCS secretary, P.O.Box 654, 2600 AR DELFT, The Netherlands

QTY REPORT TITLE

COST

Subtotal

Add postage and packing (Europe $€ 10$,-, outside Europe $€ 15,-$ )

Total

Payment by:

$\square$ Cheque/postal order * made payable to Netherlands Commission on Radiation Dosimetry (add $€ 7,50$,- for banking costs)

$\square \quad$ Please charge my Eurocard/Mastercard

Credit card No

Expiry date.

Name:

Address:

Date: Signature:

* please delete as applicable

For more information, see: http://www.ncs-dos.org/ 
This document was created with Win2PDF available at http://www.daneprairie.com. The unregistered version of Win2PDF is for evaluation or non-commercial use only. 